\title{
A universal characterization of higher algebraic $K$-theory
}

\author{
ANDREW J BLUMBERG \\ DAVID GEPNER \\ GONÇALO TABUADA
}

In this paper we establish a universal characterization of higher algebraic $K$-theory in the setting of small stable $\infty$-categories. Specifically, we prove that connective algebraic $K$-theory is the universal additive invariant, ie the universal functor with values in spectra which inverts Morita equivalences, preserves filtered colimits and satisfies Waldhausen's additivity theorem. Similarly, we prove that nonconnective algebraic $K$-theory is the universal localizing invariant, ie the universal functor that moreover satisfies the Thomason-Trobaugh-Neeman Localization Theorem.

To prove these results, we construct and study two stable $\infty$-categories of "noncommutative motives"; one associated to additivity and another to localization. In these stable $\infty$-categories, Waldhausen's $S_{\bullet}$-construction corresponds to the suspension functor and connective and nonconnective algebraic $K$-theory spectra become corepresentable by the noncommutative motive of the sphere spectrum. In particular, the algebraic $K$-theory of every scheme, stack and ring spectrum can be recovered from these categories of noncommutative motives. In the case of a connective ring spectrum $R$, we prove moreover that its negative $K$-groups are isomorphic to the negative $K$-groups of the ordinary ring $\pi_{0} R$.

In order to work with these categories of noncommutative motives, we establish comparison theorems between the category of spectral categories localized at the Morita equivalences and the category of small idempotent-complete stable $\infty$-categories. We also explain in detail the comparison between the $\infty$-categorical version of Waldhausen $K$-theory and the classical definition.

As an application of our theory, we obtain a complete classification of the natural transformations from higher algebraic $K$-theory to topological Hochschild homology $(T H H)$ and topological cyclic homology $(T C)$. Notably, we obtain an elegant conceptual description of the cyclotomic trace map.

18D20, 19D10, 19D25, 19D55, 55N15 


\section{Introduction}

Algebraic $K$-theory is a fundamental algebro-geometric invariant, capturing information in arithmetic, algebraic geometry and topology. The algebraic $K$-theory of a ring encodes many of its classical number-theoretic invariants, such as its Picard and Brauer groups. More generally, the algebraic $K$-theory of a scheme encodes arithmetic information as well as information about its singularities. The extension of algebraic $K-$ theory from classical algebraic objects to ring spectra and to derived schemes provides a connection to geometric topology; notably, Waldhausen's $A$-theory [77] (ie the $K$-theory of the sphere spectrum) is essentially equivalent to stable pseudoisotopy theory.

The subject originated with Grothendieck's definition of $K_{0}$ (the "Grothendieck group") in the course of his work on the Riemann-Roch theorem. By construction, $K_{0}$ is the universal receptacle for Euler characteristics, ie functions $\chi$ from the set of isomorphism classes of objects of a category $\mathcal{C}$ equipped with a suitable notion of "equivalence" and "exact sequence" to abelian groups which satisfy the relation

$$
\chi(X)-\chi(Y)+\chi(Z)=0
$$

whenever there is an exact sequence $X \rightarrow Y \rightarrow Z$ in $\mathcal{C}$.

During the 70s and early 80s, Quillen [59] and then Waldhausen [78] extended Grothendieck's work making use of tools from algebraic topology. They defined the connective algebraic $K$-theory spectrum $K(\mathcal{C})$ of a suitable category $\mathcal{C}$; the homotopy groups of this spectrum are the higher algebraic $K$-groups $K_{i}, i \geq 0$. Subsequently, Thomason and Trobaugh [72] generalized Bass's work [5] on negative algebraic $K-$ groups and introduced the nonconnective algebraic $K$-theory spectrum $\boldsymbol{K}(\mathcal{C})$ of $\mathcal{C}$ in order to properly capture Mayer-Vietoris phenomena for schemes.

In contrast with the Grothendieck group, however, the construction of these algebraic $K$-theory spectra does not provide a universal characterization. The most basic theorem about connective algebraic $K$-theory, the additivity theorem (see Waldhausen [78]), essentially says that the $S_{\bullet}$ construction forces the chosen cofiber sequences to split. McCarthy's simplicial proof of the additivity theorem [52] for any theory satisfying a few simple axioms suggests that the $S_{\bullet}$ construction is a universal construction for imposing additivity on a functor from categories to spectra. Moreover, the construction of the connective algebraic $K$-theory spectrum in terms of iterating the $S_{\bullet}$ construction (along with the additivity theorem applied to a simplicial path fibration) implies that the $S_{\bullet}$ construction functions as a kind of delooping functor.

However, although these shadows of a universal description have been known for a long time, a precise universal characterization of algebraic $K$-theory proved elusive. A major 
technical impediment has been the absence of a framework in which to systematically express homotopical constructions in the category of categories. This impediment has been lifted by recent developments in the foundations of higher category theory, such as the theory of derivators or $\infty$-categories.

Over the past few years the third author [68] (and Cisinski and the third author [20; 21]) have carried out a program of providing universal characterizations of algebraic $K$-theory in the setting of $\mathrm{dg}$-categories via the formalism of derivators. In this paper we adapt this approach to the setting of Lurie's theory of stable $\infty$-categories. The $\infty$-category of stable $\infty$-categories provides a natural home for many examples of interest coming from algebraic geometry and algebraic topology: the $\infty$-category of perfect complexes associated to a scheme (or a stack), the $\infty$-category of compact module spectra for a ring spectrum and the $\infty$-category of stable retractive spaces are all examples of stable $\infty$-categories.

\subsection{Universal characterization}

Let $\mathrm{Cat}_{\infty}^{\mathrm{ex}}$ be the $\infty$-category of small stable $\infty$-categories and Cat ${ }_{\infty}^{\text {perf }}$ the full subcategory of small idempotent-complete stable $\infty$-categories; see Section 2.2. An exact functor $F: \mathcal{A} \rightarrow \mathcal{B}$ in $\mathrm{Cat}_{\infty}^{\mathrm{ex}}$ is called a Morita equivalence when its idempotent completion $\operatorname{Idem}(F): \operatorname{Idem}(\mathcal{A}) \rightarrow \operatorname{Idem}(\mathcal{B})$ is an equivalence of $\infty$-categories; see Definition 4.21. This is equivalent to the condition that the induced $\operatorname{map} F_{!}: \operatorname{Mod}(\mathcal{A}) \rightarrow \operatorname{Mod}(\mathcal{B})$ is an equivalence of $\infty$-categories.

A sequence $\mathcal{A} \rightarrow \mathcal{B} \rightarrow \mathcal{C}$ in Cat ${ }_{\infty}^{\text {perf }}$ is called exact if the composite is zero, $\mathcal{A} \rightarrow \mathcal{B}$ is fully faithful and the map $\mathcal{B} / \mathcal{A} \rightarrow \mathcal{C}$, from the cofiber of the inclusion of $\mathcal{A}$ into $\mathcal{B}$ to $\mathcal{C}$, is an equivalence; see Proposition 5.15. The sequence is called split-exact if there exist adjoint splitting maps such that the relevant composites are the respective identities; see Definition 5.18. More generally, a sequence $\mathcal{A} \rightarrow \mathcal{B} \rightarrow \mathcal{C}$ in $\mathrm{Cat}_{\infty}^{\mathrm{ex}}$ is $(\operatorname{split}-)$ exact if $\operatorname{Idem}(\mathcal{A}) \rightarrow \operatorname{Idem}(\mathcal{B}) \rightarrow \operatorname{Idem}(\mathcal{C})$ is (split-)exact. Now, let

$$
E: \mathrm{Cat}_{\infty}^{\mathrm{ex}} \longrightarrow \mathcal{D}
$$

be a functor with values in a stable presentable $\infty$-category $\mathcal{D}$. We say that $E$ is an additive invariant if it inverts Morita equivalences, preserves filtered colimits and sends split-exact sequences to (split) cofiber sequences; see Definition 6.1. This last condition corresponds to the stable $\infty$-categorical analogue of Waldhausen's additivity theorem. If $E$ in fact sends all exact sequences to cofiber sequences we say that it is a localizing invariant; this property corresponds to Neeman's generalization [56] of the Thomason-Trobaugh Localization Theorem [72]. 
Every localizing invariant is additive, but not every additive invariant is necessarily localizing. Examples of localizing invariants include the nonconnective version of algebraic $K$-theory and topological Hochschild homology. Connective algebraic $K-$ theory is an example of an additive invariant which is not localizing. Note that, as we discuss in Sections 7 and 9, the $\infty$-categorical versions of these invariants agree with the "classical" definitions. For instance, we give a precise comparison in Section 7 between Waldhausen's algebraic $K$-theory of a Waldhausen category $\mathcal{C}$ and the $\infty$-categorical algebraic $K$-theory of the $\infty$-category underlying $\mathcal{C}$.

Our first main result is the following.

Theorem 1.1 (See Theorems 6.10 and 8.7) There are stable presentable $\infty$-categories $\mathcal{M}_{\text {add }}$ and $\mathcal{M}_{\text {loc }}$ and universal additive and localizing invariants

$$
\mathcal{U}_{\mathrm{add}}: \mathrm{Cat}_{\infty}^{\mathrm{ex}} \longrightarrow \mathcal{M}_{\mathrm{add}} \text { and } \mathcal{U}_{\mathrm{loc}}: \mathrm{Cat}_{\infty}^{\mathrm{ex}} \longrightarrow \mathcal{M}_{\mathrm{loc}}
$$

That is, given any stable presentable $\infty$-category $\mathcal{D}$, we have induced equivalences of $\infty$-categories

$$
\begin{aligned}
\left(\mathcal{U}_{\text {add }}\right)^{*}: \operatorname{Fun}^{\mathrm{L}}\left(\mathcal{M}_{\text {add }}, \mathcal{D}\right) \stackrel{\sim}{\longrightarrow} \operatorname{Fun}_{\text {add }}\left(\operatorname{Cat}_{\infty}^{\mathrm{ex}}, \mathcal{D}\right), \\
\left(\mathcal{U}_{\text {loc }}\right)^{*}: \operatorname{Fun}^{\mathrm{L}}\left(\mathcal{M}_{\text {loc }}, \mathcal{D}\right) \stackrel{\sim}{\longrightarrow} \operatorname{Fun}_{\text {loc }}\left(\operatorname{Cat}_{\infty}^{\mathrm{ex}}, \mathcal{D}\right),
\end{aligned}
$$

where the left-hand sides denote the $\infty$-categories of colimit-preserving functors and the right-hand sides the $\infty$-categories of additive and localizing invariants.

From a motivic perspective, the $\infty$-categories $\mathcal{M}_{\text {add }}$ and $\mathcal{M}_{\text {loc }}$ should be considered as candidate categories of noncommutative motives. In fact, Theorem 1.1 shows us that every additive (respectively localizing) invariant factors uniquely through $\mathcal{M}_{\text {add }}$ (respectively, through $\mathcal{M}_{\text {loc }}$ ). That is, all the information concerning additive (respectively, localizing) invariants is encoded in $\mathcal{M}_{\text {add }}$ (respectively, in $\mathcal{M}_{\text {loc }}$ ).

Our second main result is the following characterization of the higher algebraic $K-$ theory of a stable $\infty$-category. Any stable $\infty$-category (and in particular $\mathcal{M}_{\text {add }}$ and $\mathcal{M}_{\text {loc }}$ ) admits natural mapping spectra; that is, a stable $\infty$-category is naturally enriched over the $\infty$-category $\mathcal{S}_{\infty}$ of spectra (see Sections 2.3 and 4).

Theorem 1.3 (See Theorems 7.13 and 9.8) Let $\mathcal{A}$ be an idempotent-complete small stable $\infty$-category. Then, there are natural equivalences of spectra

$$
\begin{aligned}
\operatorname{Map}\left(\mathcal{U}_{\text {add }}\left(\mathcal{S}_{\infty}^{\omega}\right), \mathcal{U}_{\text {add }}(\mathcal{A})\right) & \simeq K(\mathcal{A}), \\
\operatorname{Map}\left(\mathcal{U}_{\text {loc }}\left(\mathcal{S}_{\infty}^{\omega}\right), \mathcal{U}_{\text {loc }}(\mathcal{A})\right) & \simeq \boldsymbol{K}(\mathcal{A}),
\end{aligned}
$$


where $\mathcal{S}_{\infty}^{\omega}$ is the small stable $\infty$-category of compact spectra. In particular, for all $n \in \mathbb{Z}$, we have isomorphisms of abelian groups

$$
\begin{aligned}
\left.\operatorname{Hom}\left(\mathcal{U}_{\text {add }}\left(\mathcal{S}_{\infty}^{\omega}\right)\right), \Sigma^{-n} \mathcal{U}_{\text {add }}(\mathcal{A})\right) & \simeq K_{n}(\mathcal{A}), \\
\left.\operatorname{Hom}\left(\mathcal{U}_{\text {loc }}\left(\mathcal{S}_{\infty}^{\omega}\right)\right), \Sigma^{-n} \mathcal{U}_{\text {loc }}(\mathcal{A})\right) & \simeq \boldsymbol{K}_{n}(\mathcal{A}),
\end{aligned}
$$

in the triangulated categories $\operatorname{Ho}\left(\mathcal{M}_{\mathrm{add}}\right)$ and $\operatorname{Ho}\left(\mathcal{M}_{\mathrm{loc}}\right)$.

Remark 1.8 In fact, stronger results are true. In equivalence (1.4), $\mathcal{S}_{\infty}^{\omega}$ can be replaced by any compact idempotent-complete small stable $\infty$-category $\mathcal{B}$ and the right-hand side by the $K$-theory spectrum of $\operatorname{Fun}^{\mathrm{ex}}(\mathcal{B}, \mathcal{A})$; see Theorem 7.13. In equivalence (1.5), $\mathcal{S}_{\infty}^{\omega}$ can be replaced by any smooth and proper (ie dualizable) small stable $\infty$-category $\mathcal{B}$ and the right-hand side by $\boldsymbol{K}\left(\mathcal{B}^{\text {op }} \widehat{\otimes} \mathcal{A}\right)$; see Theorem 9.36 .

In particular, when $\mathcal{A}$ is the $\infty$-category of perfect complexes over a suitable scheme (or stack), we recover the $K$-theory spectra of the scheme (stack). Taking $\mathcal{A}$ to be the $\infty$-category of compact modules over a ring spectrum $R$, we recover the $K$-theory of $R$. When $R$ is a connective ring spectrum, we show in Theorem 9.53 that the negative homotopy groups of the nonconnective $K$-theory of $R$ are the same as those of $\pi_{0} R$. However, we expect the nonconnective $K$-theory of a nonconnective ring spectrum to be an interesting new invariant.

The left-hand sides of the natural equivalences and isomorphisms of Theorem 1.3 are defined solely in terms of universal constructions on presheaf categories; algebraic $K_{-}$ theory is not used in their construction. Rather, a variant of Waldhausen's path-fibration argument (see Proposition 7.17) shows that Waldhausen's $S_{\bullet}$ construction acts as the suspension functor in $\mathcal{M}_{\text {add }}$ and also $\mathcal{M}_{\text {loc }}$.

Therefore, Theorem 1.3 (combined with Theorem 1.1) provides an intrinsic characterization of algebraic $K$-theory as a functor of stable $\infty$-categories. Furthermore, Theorem 1.12 below (see also Theorem 10.3 in the text) shows that the corepresentability result coupled with the Yoneda Lemma provides a complete classification of all natural transformations from algebraic $K$-theory to an arbitrary additive (or localizing) functor from small stable categories to spectra.

Remark 1.9 Analogues of Theorems 1.1, 1.3 and 1.12 in the setting of dg-categories were previously known (due to the third author and Cisinski $[20 ; 21 ; 68]$ ). Our arguments follow the same general outline. 


\subsection{Morita theory}

The main technical device in our proofs of Theorems 1.1 and 1.3 is the Morita theory of stable categories and spectral categories. In particular, we prove Theorem 1.3 by using a comparison result between the theory of small spectral categories (see Section 2.1) and the theory of small stable $\infty$-categories to rigidify questions about the algebraic $K$-theory of $\infty$-categories to corresponding questions in the (classical) Waldhausen $K$-theory of Waldhausen categories.

The category Cat $\mathcal{S}$ of small spectral categories carries a Quillen model category structure in which the weak equivalences are the $D K$-equivalences, ie the functors that are fully faithful and essentially surjective up to weak homotopy equivalence; see the third author's [69] (reprised below in Theorem 2.2). As a consequence, we can form the associated $\infty$-category $\left(\mathrm{Cat}_{\mathcal{S}}\right)_{\infty}$ of small spectral categories.

A spectral functor $F: \mathcal{A} \rightarrow \mathcal{B}$ is called a triangulated equivalence if it induces a weak equivalence on the triangulated closures of $\mathcal{A}$ and $\mathcal{B}$, and it is called a Morita equivalence if it induces a weak equivalence on the thick closures of $\mathcal{A}$ and $\mathcal{B}$; see Definition 2.7. Our comparison result, which can be regarded as a generalization of the Morita theory of [65], is the following.

Theorem 1.10 (See Theorems 4.22 and 4.23) The accessible localization of $\left(\mathrm{Cat}_{\mathcal{S}}\right)_{\infty}$ along the triangulated equivalences is equivalent to $\mathrm{Cat}_{\infty}^{\mathrm{ex}}$, and the (further) localization of $\left(\mathrm{Cat}_{\mathcal{S}}\right)_{\infty}$ along the Morita equivalences is equivalent to $\mathrm{Cat}_{\infty}^{\mathrm{perf}}$.

We use this comparison result to deduce structural properties of the categories $\mathrm{Cat}_{\infty}^{\mathrm{ex}}$ and $\mathrm{Cat}_{\infty}^{\text {perf }}$, notably that they are compactly generated, complete and cocomplete; see Corollary 4.25. Furthermore, we prove Theorem 1.3 by using Theorem 1.10 to lift split-exact sequences of small $\infty$-categories to split-exact sequences of small spectral categories so we can take the $K$-theory in the setting of Waldhausen categories. More generally, this comparison result explains the relationship between the classical versions of algebraic $K$-theory (and topological Hochschild and cyclic homology) and the $\infty$-categorical versions. We believe that Theorem 1.10 is of independent interest and expect it will find applications in the future. For instance, this theorem provides clean and concise proofs of the main theorems of Toën's work [73] on internal hom objects in the category of dg-categories and its (previously unknown) extension to the context of spectral categories.

\subsection{Symmetric monoidal structure and dualizable objects}

The category Cat ${ }_{\infty}^{\text {perf }}$ is a symmetric monoidal $\infty$-category (in the sense of Lurie [48, Section 2]), in which the tensor product $\widehat{\otimes}$ is characterized by the property that maps 
out of $\mathcal{A} \widehat{\otimes} \mathcal{B}$ correspond to maps out of the product $\mathcal{A} \times \mathcal{B}$ that preserve colimits in each variable; see Section 3 for a discussion of this structure, following the work of Lurie [48] and Ben-Zvi, Francis and Nadler [6]. We will reserve a careful study of the structure of $\mathcal{M}_{\text {add }}$ and $\mathcal{M}_{\text {loc }}$ as symmetric monoidal $\infty$-categories for the authors' forthcoming paper [9]. However, in order to carry out the extension of the nonconnective corepresentability theorem described in Remark 1.8, we study the theory of dualizable objects in $\mathrm{Cat}_{\infty}^{\text {perf }}$, using the theory of [48, Section 4.2.5].

In analogy with the situation for dg-categories [21, Section 4], we obtain a characterization of the dualizable objects in $\mathrm{Cat}_{\infty}^{\mathrm{perf}}$ as the smooth and proper objects. We define these notions as follows. Implicit in the comparison between small stable $\infty-$ categories and spectral categories of Theorem 1.10 is the fact that for objects $a$ and $b$ in a small stable $\infty$-category $\mathcal{A}$ there exists a natural mapping spectrum $\mathcal{A}(a, b)$ (see Definition 2.15). Using this fact, we say that a small stable $\infty$-category $\mathcal{A}$ is proper if, for all pairs of objects $a$ and $b$ of $\mathcal{A}$, the mapping spectrum $\mathcal{A}(a, b)$ is compact. We say that a small stable $\infty$-category $\mathcal{A}$ is smooth if it is perfect as an $\mathcal{A}^{\mathrm{op}} \widehat{\otimes} \mathcal{A}$-module. (Here we use the fact that any small stable $\infty$-category can be regarded as a bimodule over itself.) We then have the following theorem characterizing the dualizable objects in these terms:

Theorem 1.11 (See Theorem 3.7) An idempotent-complete small stable $\infty$-category $\mathcal{A}$ is dualizable (as an object of the symmetric monoidal $\infty$-category $\mathrm{Cat}_{\infty}^{\mathrm{perf}}$ ) if and only if $\mathcal{A}$ is smooth and proper. Moreover, the dual of a dualizable idempotent-complete small stable $\infty$-category $\mathcal{A}$ is the opposite $\infty$-category $\mathcal{A}^{\mathrm{op}}$.

\subsection{Trace methods}

One of the major revolutions in the calculational study of algebraic $K$-theory of rings and schemes in the past two decades has been the development of trace methods, following the ideas of Goodwillie [35] and Bokstedt-Hsiang-Madsen [17]. The cyclotomic trace from $K$-theory to topological cyclic homology $T C$ and topological Hochschild homology $\mathrm{THH}$ (stable homotopy theory generalizations of negative cyclic homology and Hochschild homology) has allowed major calculational advances. The fiber of this map is well understood by work of Goodwillie [35], McCarthy [53] and Dundas [26], and the target is relatively computable using the methods of equivariant stable homotopy theory (eg see the extensive body of work by Hesselholt and Madsen on the Quillen-Lichtenbaum conjecture [37]). One application of the corepresentability of algebraic $K$-theorem (Theorem 1.3) is the complete classification of all natural transformations with source the algebraic $K$-theory functor. 
Theorem 1.12 (See Theorem 10.3) Given an additive invariant

$$
E: \mathrm{Cat}_{\infty}^{\mathrm{ex}} \longrightarrow \mathcal{S}_{\infty}
$$

with values in the stable $\infty$-category of spectra, we have a natural equivalence of spectra

$$
\operatorname{Map}(K, E) \simeq E\left(\mathcal{S}_{\infty}^{\omega}\right),
$$

where $\operatorname{Map}(K, E)$ denotes the spectrum of natural transformations of additive invariants. The analogous result for localizing invariants holds. In the particular case where $E$ is topological Hochschild homology, we obtain an isomorphism

$$
\pi_{0} \operatorname{Map}(K, T H H) \simeq \pi_{0} T H H\left(\mathcal{S}_{\infty}^{\omega}\right) \simeq \pi_{0} T H H(\mathbb{S}) \simeq \mathbb{Z} .
$$

A calculation then provides a canonical construction and conceptual description of the topological Dennis trace map $K \rightarrow T H H$.

Corollary 1.13 (See Section 10) The set of homotopy classes of natural transformations of additive invariants from connective algebraic $\mathrm{K}$-theory to $\mathrm{THH}$ is isomorphic to $\mathbb{Z}$; furthermore, the topological Dennis trace is characterized up to homotopy as the natural transformation $K \rightarrow T H H$ corresponding to the unit $1 \in \mathbb{Z}$.

That is, up to scaling, the trace is the only natural transformation of additive invariants between connective algebraic $K$-theory and $T H H$. This provides a direct proof that all known constructions of the topological Dennis trace map agree up to homotopy.

Working directly with topological cyclic homology $(T C)$ is somewhat more complicated; $T C$ does not preserve filtered colimits in general, and is therefore not an additive or localizing functor. However, we deduce an analogous identification of the cyclotomic trace as determined by the unit map; see Section 10 .

Finally, we note that in the localizing setting our results provide an extension of the cyclotomic trace from nonconnective algebraic $K$-theory to the nonconnective versions of $T C$ and $T H H$. This generalizes and extends the nonconnective traces constructed by Geisser and Hesselholt [33] and by the first author and Mandell [14] for rings and schemes.

\subsection{Related works}

The "motivic" idea of constructing universal invariants is not new and appears in several different subjects: for example, Cortiñas-Thom's work [22] on bivariant algebraic $K-$ theory, Higson's work [38] on Kasparov's bivariant $K$-theory, Meyer-Nest's work [54] 
on $C^{*}$-algebras, Morel-Voevodsky's work [55] on $\mathbb{A}^{1}$-homotopy theory of schemes and Voevodsky's work [75] on (mixed) motives.

In this vein, over the past few years the third author and Cisinski have carried out a program $[20 ; 21 ; 68]$ of providing universal characterizations of algebraic $K$-theory in the setting of dg-categories, using the formalism of derivators. One of the main goals of our work in this paper completes this program by extending these results to stable $\infty$-categories and by solving a key open question left open in the previous work of the third author, namely identifying the cyclotomic trace in terms of the corepresentability results.

Finally, we would like to mention that Barwick [2] has recent work on a universal characterization of higher algebraic $K$-theory, in the context of a detailed study of the algebraic $K$-theory of $\infty$-categories.

Acknowledgments The authors would like to thank Mike Mandell for many helpful conversations and Haynes Miller for asking motivating questions. They are grateful to Ben Antieau, David Ben-Zvi, Chris Brav, Bob Bruner, Jonathan Campbell, Denis-Charles Cisinski, Bjorn Dundas, Tom Goodwillie, Jeremiah Heller, Kathryn Hess, John Lind, Peter May, Jack Morava, Markus Spitzweck and Bertrand Toën for helpful comments on a previous draft. The authors would like also to thank the anonymous referees for very careful readings and detailed comments and corrections which substantially improved this paper. This project was initiated during a visit by the second and third authors to Stanford's math department, and they would like to thank the department for its hospitality. Finally, the authors would like to thank the Midwest Topology Network for funding various trips which facilitated the conduct of this research. A J Blumberg was supported in part by NSF grant number DMS-0906105. G Tabuada was partially supported by the NEC award-2742738, by the Estimulo à Investigação Award 2008 - Calouste Gulbenkian Foundation, and by the Portugese Foundation for Science and Technology through the grants PTDC/MAT/098317/2008 and Pest-OE/MAT/UI0297/2011 (CMA).

\section{Spectral categories and stable $\infty$-categories}

The purpose of this section is to collect and recall the results about spectral categories and stable $\infty$-categories we will require for our constructions.

\subsection{Review of spectral categories}

We write $\mathcal{T}$ for the symmetric monoidal simplicial model category of simplicial sets and $\mathcal{S}$ for the symmetric monoidal simplicial model category of symmetric spectra [41]. 
Recall that a spectral category $\mathcal{A}$ is a category enriched in the category of symmetric spectra. Specifically, a spectral category is given by:

- A class of objects $\operatorname{obj}(\mathcal{A})$.

- For each pair of objects $(x, y)$ of $\mathcal{A}$, a symmetric spectrum $\mathcal{A}(x, y)$.

- For each triple of objects $(x, y, z)$ of $\mathcal{A}$, a composition morphism in $\mathcal{S}$

$$
\mathcal{A}(y, z) \wedge \mathcal{A}(x, y) \longrightarrow \mathcal{A}(x, z),
$$

satisfying the usual associativity condition.

- For any object $x$ of $\mathcal{A}$, a morphism $\mathbb{S} \rightarrow \mathcal{A}(x, x)$ in $\mathcal{S}$, satisfying the usual unit condition with respect to the above composition.

A spectral category is said to be small if its class of objects forms a (small) set. We write $\mathrm{Cat}_{\mathcal{S}}$ the category of small spectral categories and spectral (enriched) functors. References on spectral categories are [14, Section 2], [65, Appendix A] and [69, Section 2].

We now briefly recall the Quillen model structure on spectral categories we work with in this paper. Given a spectral category $\mathcal{A}$, we can form a genuine category $[\mathcal{A}]$ by keeping the same set of objects and defining the set of morphisms between $x$ and $y$ in $[\mathcal{A}]$ to be the set of morphisms in the homotopy category $\operatorname{Ho}(\mathcal{S})$ from the sphere spectrum $\mathbb{S}$ to $\mathcal{A}(x, y)$. We obtain in this way a functor

$$
[-]: \mathrm{Cat}_{\mathcal{S}} \longrightarrow \text { Cat, }
$$

with values in the category of small categories. Equivalently, we can think of $[-]$ as computed by passing to $\pi_{0}$ on the morphism spectra, and so we will also refer to $[\mathcal{A}]$ as the homotopy category $\operatorname{Ho}(\mathcal{A})$.

Definition 2.1 A spectral functor $F: \mathcal{A} \rightarrow \mathcal{B}$ is a $D K$-equivalence if:

- For all objects $x, y \in \mathcal{A}$, the morphism in $\mathcal{S}$

$$
F(x, y): \mathcal{A}(x, y) \longrightarrow \mathcal{B}(F x, F y)
$$

is a stable equivalence.

- The induced functor

$$
[F]:[\mathcal{A}] \longrightarrow[\mathcal{B}]
$$

is an equivalence of categories.

Theorem 2.2 $[69,5.10]$ The category $\mathrm{Cat}_{\mathcal{S}}$ carries a right proper Quillen model structure whose weak equivalences are the DK-equivalences. 
Recall from $[69, \S 2]$ the natural adjunction

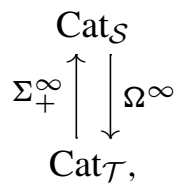

between spectral and simplicial categories, where $\Omega^{\infty}$ (also denoted $\left.(-)_{0}\right)$ is the space of maps from the unit (equivalently, restriction to the 0 -th space of the spectrum). We will use this adjunction to pass between spectral categories and $\infty$-categories. Using the model structure on simplicial categories of [7], the pair $\left(\Sigma_{+}^{\infty}, \Omega^{\infty}\right)$ is a Quillen adjunction.

For technical control, we require the following corollary which sharpens the description of the model structure, providing a combinatorial model category. (For references for Jeff Smith's theory of combinatorial model categories, see [3] or [24].)

Corollary 2.4 The category $\mathrm{Cat}_{\mathcal{S}}$ endowed with the model structure of Theorem 2.2 is a combinatorial model category and is Quillen equivalent (via a zig-zag) to a simplicial category with a left proper combinatorial simplicial model structure. There are simplicial cofibrant and fibrant replacement functors. The adjunction $\left(\Sigma_{+}^{\infty}, \Omega^{\infty}\right)$ can be lifted to a simplicial Quillen adjunction.

Proof The proof of this corollary follows from a refinement of the proof of Theorem 2.2. The model structure therein arises as the Bousfield localization of a cofibrantly generated model structure on $\mathrm{Cat}_{\mathcal{S}}$ in which the weak equivalences are the levelwise equivalences [69, Section 4], ie the spectral functors $F: \mathcal{A} \rightarrow \mathcal{B}$ such that for all objects $x, y \in \mathcal{A}$, the morphism $\mathcal{A}(x, y) \rightarrow \mathcal{B}(F x, F y)$ is a levelwise equivalence and the induced simplicial functor $\Omega^{\infty}(\mathcal{A}) \rightarrow \Omega^{\infty}(\mathcal{B})$ is a DK-equivalence.

First, we observe that the category $\mathrm{Cat}_{\mathcal{S}}$ is locally presentable; a small set of small generators is given by applying the functor $U$ (see [69, A.1]) to a small set of small generators for the category of symmetric spectra. Since the levelwise model structure on $\mathrm{Cat}_{\mathcal{S}}$ is cofibrantly generated, it follows that it is combinatorial. Next, the arguments of [69] produce a generating small set of DK-equivalences at which to localize $\mathrm{Cat}_{\mathcal{S}}$. The main theorem about the existence of left Bousfield localization for combinatorial model categories (eg see the treatment in [3]) now implies that we can localize and obtain a combinatorial model structure on $\mathrm{Cat}_{\mathcal{S}}$.

The machinery of Dugger's approach to universal homotopy theories [24] now permits us to replace $\mathrm{Cat}_{\mathcal{S}}$ with a Quillen equivalent simplicial model category (the simplicial objects over $\mathrm{Cat}_{\mathcal{S}}$ ) which is combinatorial and left proper. By applying the techniques 
of [24] and [61], we can promote this adjunction to a simplicial Quillen adjunction. Specifically, the simplicial prolongation of the adjunction forms a Quillen pair on the categories of simplicial objects $[61,6.1]$.

Let $\mathcal{A}$ be a (fixed) small spectral category and let $\mathcal{A}^{\text {op }}$ denote the opposite spectral category, defined by $\mathcal{A}^{\mathrm{op}}(x, y)=\mathcal{A}(y, x)$.

Definition 2.5 A $\mathcal{A}$-module is a spectral functor from $\mathcal{A}^{\text {op }}$ to the spectral category $\mathcal{S}$ of symmetric spectra. We denote by $\widehat{\mathcal{A}}$ the spectral category of $\mathcal{A}$-modules.

By [65, A.1.1], $\widehat{\mathcal{A}}$ can be given a combinatorial spectral model structure in which the weak equivalences are the pointwise stable equivalences and the fibrations are pointwise fibrations (referred to as the projective model structure). We will denote by $\widehat{\mathcal{A}}^{\text {cf }}$ the full spectral subcategory of $\widehat{\mathcal{A}}$ on the cofibrant and fibrant $\mathcal{A}$-modules, and by $\mathcal{D}(\mathcal{A})$ the derived category of $\mathcal{A}$, ie the homotopy category $\operatorname{Ho}(\widehat{\mathcal{A}})$ associated to the model structure. As usual, there is an equivalence $\left[\widehat{\mathcal{A}}^{\mathrm{cf}}\right] \simeq \mathcal{D}(\mathcal{A})$.

Notice that we have a (fully faithful) spectral Yoneda embedding $\mathcal{A} \rightarrow \widehat{\mathcal{A}}$ which sends the object $z$ to the functor $\mathcal{A}(-, z): \mathcal{A}^{\text {op }} \rightarrow \mathcal{S}$ represented by $z$. Note that when $\mathcal{A}$ is fibrant, the Yoneda embedding lands in $\widehat{\mathcal{A}}^{\text {cf }}$. By [65, §A.1], a spectral functor $F: \mathcal{A} \rightarrow \mathcal{B}$ gives rise to a restriction/extension Quillen adjunction

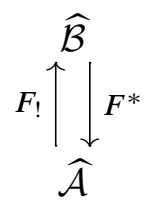

and therefore a total left-derived functor $\mathbb{L} F_{!}: \mathcal{D}(\mathcal{A}) \rightarrow \mathcal{D}(\mathcal{B})$.

We will be most interested in spectral categories $\mathcal{A}$ for which the homotopy category $\operatorname{Ho}(\mathcal{A})$ has a triangulated structure compatible with the mapping spectra; we refer to [14, 4.4] for the definition of a pretriangulated spectral category, and highlight the essential consequence $[14,4.6]$ that the homotopy category of a pretriangulated spectral category is triangulated (with distinguished triangles given by the Puppe sequences). This is the stable homotopy theory analogue of the notion of a pretriangulated dg-category. A spectral functor between pretriangulated spectral categories is a DK-equivalence if and only if it induces an equivalence on homotopy categories [10, 5.7]. Using the Yoneda embedding, we can construct minimal pretriangulated categories containing the spectral category $\mathcal{A}$.

Given a spectral category $\mathcal{A}$, the proof of $[14,4.5]$ constructs a functorial "triangulated closure" $\widehat{\mathcal{A}}_{\text {tri }}$ which is a pretriangulated spectral category. Briefly, $\widehat{\mathcal{A}}_{\text {tri }}$ consists of the 
subcategory of cofibrant-fibrant objects in $\widehat{\mathcal{A}}$ which have the homotopy type of finite cell objects (in the projective model structure). Using retracts of finite cell objects instead $[14,4.5]$ produces a functorial "thick closure" $\widehat{\mathcal{A}}_{\text {perf }}$, which is an idempotent-complete pretriangulated spectral category.

Remark 2.6 In order for the preceding definitions to produce small spectral categories, we need to restrict the sizes of the sets in the spaces of the mapping spectra. A careful discussion of this issue appears in [14, Section 4]; see also [11]. We return to the issue of set-theoretic considerations in Section 2.4.

We start with a spectral functor $F: \mathcal{A} \rightarrow \mathcal{B}$, and tacitly assume we have performed a functorial fibrant replacement. We denote by $F_{!}^{\text {cf }}: \widehat{\mathcal{A}}^{\text {cf }} \rightarrow \widehat{\mathcal{B}}^{\text {cf }}$ the composite of $F_{!}$ with a fibrant replacement (note that we do not need a cofibrant replacement here since

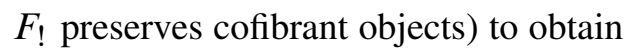

$$
F_{!}^{\mathrm{cf}}: \widehat{\mathcal{A}}^{\mathrm{cf}} \longrightarrow \widehat{\mathcal{B}}^{\mathrm{cf}} \text {. }
$$

Since this is a model of the derived functor of $F_{\text {! }}$ as a left Quillen functor, it preserves homotopy colimits and thus sends modules of the homotopy type of finite cell $\mathcal{A}$ modules to modules of the homotopy type of finite cell $\mathcal{B}$-modules and hence perfect $\mathcal{A}$-modules to perfect $\mathcal{B}$-modules. Therefore, the following definitions make sense.

Definition 2.7 A spectral functor $F: \mathcal{A} \rightarrow \mathcal{B}$ is called

- a triangulated equivalence if the induced functor

$$
F_{!}^{\text {cf. }} \widehat{\mathcal{A}}_{\text {tri }} \longrightarrow \widehat{\mathcal{B}}_{\text {tri }}
$$

is a DK-equivalence of spectral categories;

- a Morita equivalence if the induced functor

$$
F_{!}^{\mathrm{cf}}: \widehat{\mathcal{A}}_{\text {perf }} \longrightarrow \widehat{\mathcal{B}}_{\text {perf }}
$$

is a DK-equivalence of spectral categories.

Remark 2.8 Suppose we are given a spectral functor $F: \mathcal{A} \rightarrow \mathcal{B}$. Since $\widehat{\mathcal{A}}^{\text {cf }}$ is generated by $\widehat{\mathcal{A}}_{\text {perf }}$ under filtered homotopy colimits and Morita equivalences are stable under filtered homotopy colimits, it follows that $F$ is a Morita equivalence if and only if $F_{!}^{\mathrm{cf}}: \widehat{\mathcal{A}}^{\mathrm{cf}} \rightarrow \widehat{\mathcal{B}}^{\mathrm{cf}}$ is a DK-equivalence.

We can relate these notions to definitions purely on the level of triangulated categories (the relationship between triangulated constructions and enriched constructions is discussed further in Section 5). For a spectral category $\mathcal{A}$, let $\mathcal{D}_{\text {tri }}(\mathcal{A})$ denote the smallest 
triangulated subcategory of $\mathcal{D}(\mathcal{A})$ containing the image of the $\mathcal{A}$ under the Yoneda embedding, and $\mathcal{D}_{\text {perf }}(\mathcal{A})$ denote the smallest thick subcategory of $\mathcal{D}(\mathcal{A})$ containing the image of $\mathcal{A}$ under the Yoneda embedding. Observe that $\mathcal{D}_{\text {tri }}(\mathcal{A}) \simeq \operatorname{Ho}\left(\widehat{\mathcal{A}}_{\text {tri }}\right)$ and $\mathcal{D}_{\text {perf }}(\mathcal{A}) \simeq \operatorname{Ho}\left(\widehat{\mathcal{A}}_{\text {perf }}\right)$. As a consequence, we obtain the following proposition.

Proposition 2.9 A spectral functor $F: \mathcal{A} \rightarrow \mathcal{B}$ is

- a triangulated equivalence if and only if the induced derived functor

$$
\mathbb{L} F_{!}: \mathcal{D}_{\text {tri }}(\mathcal{A}) \longrightarrow \mathcal{D}_{\text {tri }}(\mathcal{B})
$$

is an equivalence of (triangulated) categories;

- a Morita equivalence if and only if the induced derived functor

$$
\mathbb{L} F_{!}: \mathcal{D}_{\text {perf }}(\mathcal{A}) \longrightarrow \mathcal{D}_{\text {perf }}(\mathcal{B})
$$

is an equivalence of (triangulated) categories.

Proof This follows immediately from $[10,5.7]$.

Finally, note that we can use $\Omega^{\infty}$ to obtain simplicial models of the triangulated and thick closures. Define $\operatorname{Mod}(\mathcal{A})$ to be the simplicial category $\Omega^{\infty} \widehat{\mathcal{A}}^{\text {cf }}, \operatorname{Mod}(\mathcal{A})_{\text {tri }}$ to be the simplicial category $\Omega^{\infty} \widehat{\mathcal{A}}_{\text {tri }}$, and $\operatorname{Mod}(\mathcal{A})_{\text {perf }}$ to be the simplicial category $\Omega^{\infty} \widehat{\mathcal{A}}_{\text {perf }}$. Of course, it is also possible to give intrinsic definitions of the latter two categories in terms of $\operatorname{Mod}(\mathcal{A})$.

Summarizing the relationships between the various categories, we have the following commutative diagram (with horizontal arrows induced by the Yoneda embedding and subsequent inclusions):

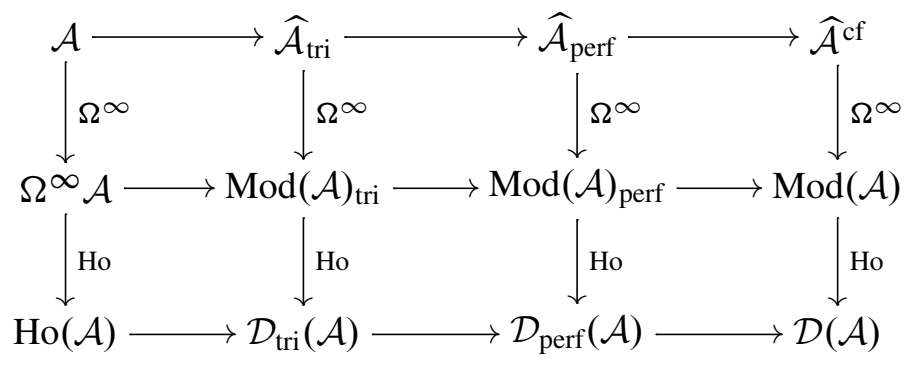




\subsection{The $\infty$-categories $\mathrm{Cat}_{\infty}^{\mathrm{ex}}$ and $\mathrm{Cat}_{\infty}^{\mathrm{perf}}$}

The basic setting for our work is the theory of $\infty$-categories (and particularly stable $\infty$-categories), which provide a tractable way to handle a "homotopical category of homotopical categories" as well as homotopically meaningful categories of homotopical functors. There are now many competing models of $\infty$-categories, including Rezk's complete Segal spaces [60], the Segal categories of Simpson (see Hirschowitz and Simpson [40]) and Tamsamani [71], the quasicategories (weak Kan complexes) of Boardman and Vogt, the homotopy theory of simplicial categories as studied by DwyerKan [28] and Bergner [7] and others, all of which are known to be equivalent (see [8] for a nice discussion of the situation). In a sense the situation is analogous to the situation with the varied modern categories of spectra (eg symmetric spectra, orthogonal spectra, EKMM $S$-modules). None of the work of this paper depends in any way on particular properties of the model of $\infty$-categories chosen; given certain basic structural properties, one could carry out our arguments in any of them.

We have chosen to work in this paper with the theory of quasicategories. These first appeared in the work of Boardman and Vogt [15], where they were referred to as weak Kan complexes. The theory was subsequently developed by Joyal [42] and then extensively studied by Lurie. In this section we give a rapid review of the relevant background on the theory of quasicategories as a model of $\infty$-categories. Our basic references for this material are Lurie's books [48; 47].

We will write Cat $\infty$ to denote the $\infty$-category of small $\infty$-categories and functors, which we explicitly model as the category of simplicial sets with the Joyal model structure [42]. There is a simplicial nerve functor $\mathrm{N}$ from simplicial categories to simplicial sets which is the right Quillen functor of a Quillen equivalence [47, Section 1.1.5.5, 1.1.5.13, 2.2.5.1]:

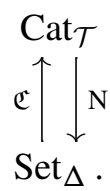

Here the model structure on the top is Bergner-Dwyer-Kan's model structure on simplicial categories [7] and the model structure on the bottom is Joyal's model structure on simplicial sets.

There are a number of options for producing the "underlying" $\infty$-category of a category equipped with a notion of "weak equivalence". The most structured setting is that of a simplicial model category $\mathcal{C}$, where the $\infty$-category can be obtained by restricting to the full simplicial subcategory $\mathcal{C}^{\mathrm{cf}}$ of cofibrant-fibrant objects and then 
applying the simplicial nerve functor $\mathrm{N}$. More generally, if $\mathcal{C}$ is a category equipped with a subcategory of weak equivalences $w \mathcal{C}$, the Dwyer-Kan simplicial localization $L \mathcal{C}$ [28] provides a corresponding simplicial category, and then $\mathrm{N}\left((L \mathcal{C})^{\mathrm{fib}}\right)$, where $(-)^{\mathrm{fib}}$ denotes fibrant replacement in simplicial categories, yields an associated $\infty-$ category. Barwick and Kan [4] have studied this procedure in the context of Segal spaces and Lurie has given a version of this approach in [48, Section 1.3.4]: we associate to a (not necessarily simplicial) category $\mathcal{C}$ with weak equivalences $W$ an $\infty$-category $\mathrm{N}(\mathcal{C})\left[W^{-1}\right]$; when $\mathcal{C}$ is a model category, for functoriality reasons it is usually convenient to restrict to the cofibrant objects $\mathcal{C}^{\mathrm{c}}$ and consider $\mathrm{N}\left(\mathcal{C}^{\mathrm{c}}\right)\left[W^{-1}\right]$.

All of these constructions produce equivalent $\infty$-categories [48, 1.3.4.20]. Furthermore, all of them are functorial. Although a simplicial left Quillen functor $\mathcal{C} \rightarrow \mathcal{D}$ does not typically induce a functor between the subcategories of cofibrant-fibrant objects in $\mathcal{C}$ and $\mathcal{D}$ respectively, composing with a fibrant replacement functor as in Definition 2.7, does yield an induced functor on simplicial nerves. Furthermore, given a simplicial Quillen adjunction $(F, G)$, there is an induced adjunction of functors on the level of $\infty$-categories by $[47,5.2 .4 .6]$. (Alternatively, it can be seen directly that a functor $\mathcal{C} \rightarrow \mathcal{D}$ which preserves weak equivalences between cofibrant objects induces a functor $\mathrm{N}(\mathcal{C})\left[W^{-1}\right] \rightarrow \mathrm{N}(\mathcal{D})\left[W^{-1}\right]$.)

Given an $\infty$-category $\mathcal{C}$, we can form its homotopy category $\operatorname{Ho}(\mathcal{C})$, which is an ordinary category [47, Section 1.2.3]. In addition, given an $\infty$-category $\mathcal{C}$, there is a maximal $\infty$-groupoid (Kan complex) $\mathcal{C}_{\text {iso }}$ inside of $\mathcal{C}$, obtained by restricting to the subcategory of $\mathcal{C}$ consisting of those arrows which become isomorphisms in the homotopy category $\operatorname{Ho}(\mathcal{C})$. The functor which associates to the $\infty$-category $\mathcal{C}$ its maximal subgroupoid $\mathcal{C}_{\text {iso }}$ is right adjoint to the inclusion of $\infty$-groupoids into $\infty$-categories. We have the following proposition relating this to other, possibly more familiar, notions (see also [74, 2.3]).

Proposition 2.10 Let $\mathcal{C}$ be a small category with a subcategory $w \mathcal{C}$ of weak equivalences which satisfies a homotopy calculus of two-sided fractions (in the sense of Dwyer and Kan [27, 6.1]). Then there is a weak equivalence of simplicial sets

$$
\mathrm{N}(w \mathcal{C}) \simeq\left(\mathrm{N}\left(\left(L^{H} \mathcal{C}\right)^{\mathrm{fib}}\right)\right)_{\text {iso }},
$$

where here $L^{H} \mathcal{C}$ denotes the hammock version of the simplicial localization [27] and $\left(L^{H} \mathcal{C}\right)^{\mathrm{fib}}$ is a fibrant replacement of $L^{H} \mathcal{C}$ as a simplicial category.

Proof There is an "inclusion" functor $\mathcal{C} \rightarrow L^{H} \mathcal{C}$. Restricting to the weak equivalences and passing to nerves via $\mathrm{N}$, we obtain a map of simplicial sets

$$
\mathrm{N}(w \mathcal{C}) \rightarrow \mathrm{N}\left(L^{H} w \mathcal{C}\right) \rightarrow \mathrm{N}\left(\left(L^{H} w \mathcal{C}\right)^{\mathrm{fib}}\right)
$$


note that the nerve of $w \mathcal{C}$ is the same whether we regard it as a category or as a category (trivially) enriched in simplicial sets [47, 1.1.5.8]. Since $\left(\mathrm{N}\left(\left(L^{H} w \mathcal{C}\right)^{\mathrm{fib}}\right)\right)$ iso is isomorphic to $\mathrm{N}\left(\left(L^{H} w \mathcal{C}\right)^{\mathrm{fib}}\right)$, the inclusion $L^{H} w \mathcal{C} \rightarrow L^{H} \mathcal{C}$ induces a natural map $\mathrm{N}\left(\left(L^{H} w \mathcal{C}\right)^{\mathrm{fib}}\right) \rightarrow\left(\mathrm{N}\left(\left(L^{H} \mathcal{C}\right)^{\mathrm{fib}}\right)\right)_{\text {iso }}$; under the hypothesis that $\mathcal{C}$ satisfies a homotopy calculus of fractions, this map is a weak equivalence [27, 6.4]. Therefore, it suffices to show that the map of Equation (2.11) is a weak equivalence. We consider the map on components; for each homotopy equivalence class $[x]$, both sides are equivalent to $B$ haut $(x)$ and it is straightforward to see that the map induces the equivalence.

The $\infty$-category of functors between two $\infty$-categories $\mathcal{C}$ and $\mathcal{D}$ is denoted $\operatorname{Fun}(\mathcal{C}, \mathcal{D})$. As a point-set object, in this setting $\operatorname{Fun}(\mathcal{C}, \mathcal{D})$ is the simplicial set of maps between the quasicategories $\mathcal{C}$ and $\mathcal{D}$, which is itself a quasicategory. Note that the space of functors from $\mathcal{C}$ to $\mathcal{D}$ is precisely the maximal subgroupoid $\operatorname{Fun}(\mathcal{C}, \mathcal{D})_{\text {iso }}$ of $\operatorname{Fun}(\mathcal{C}, \mathcal{D})$ $[47,1.2 .5 .3,3.0 .0 .1]$.

Definition 2.12 An $\infty$-category is stable [48, 1.1.1.9] if it has finite limits and colimits and pushout and pullback squares coincide [48, 1.1.3.4]. Let Cat ${ }_{\infty}^{\mathrm{ex}}$ denote the (pointed) $\infty$-category of small stable $\infty$-categories and exact functors (ie functors which preserve finite limits and colimits) [48, Section 1.1.4]. The $\infty$-category of exact functors between $\mathcal{A}$ and $\mathcal{B}$ is denoted by $\operatorname{Fun}^{\mathrm{ex}}(\mathcal{A}, \mathcal{B})$; this is the full $\infty$-subcategory of $\operatorname{Fun}(\mathcal{A}, \mathcal{B})$ spanned by the exact functors.

For a small stable $\infty$-category $\mathcal{C}$, the homotopy category $\operatorname{Ho}(\mathcal{C})$ is triangulated, with the exact triangles determined by the cofiber sequences in $\mathcal{C}$ [48, 1.1.2.14].

Remark 2.13 A small stable $\infty$-category corresponds to the notion of a pretriangulated spectral category, and the weak equivalences are given by exact functors which induce triangulated equivalences on passage to the homotopy category. We will make this correspondence precise in Section 4, but for now observe that given a pretriangulated spectral category $\mathcal{C}$, the $\infty$-category $\mathrm{N}\left((\operatorname{Mod}(\mathcal{C}))^{\text {cf }}\right)$ is stable. Recall that a stable model category is a pointed model category $\mathcal{C}$ for which the functors $\Sigma$ and $\Omega$ on $\operatorname{Ho}(\mathcal{C})$ are inverse equivalences. Given a stable simplicial model category $\mathcal{C}$, the $\infty$-category $\mathrm{N}\left(\mathcal{C}^{\text {cf }}\right)$ is stable. More generally, if $\mathcal{C}$ is a stable model category, $\mathrm{N}\left(\mathcal{C}^{\mathrm{c}}\right)\left[W^{-1}\right]$ is a stable $\infty$-category.

Recall that an $\infty$-category $\mathcal{C}$ is idempotent-complete if the image of $\mathcal{C}$ under the Yoneda embedding $\mathcal{C} \rightarrow \operatorname{Pre}(\mathcal{C})$ is closed under retracts (see also [47, Section 4.4.5]); here $\operatorname{Pre}(\mathcal{C})$ denotes the $\infty$-category $\operatorname{Fun}\left(\mathcal{C}^{\text {op }}, \mathrm{N}\left(\mathcal{T}^{\text {cf }}\right)\right)$ of presheaves of spaces on $\mathcal{C}$. Let $\mathrm{Cat}_{\infty}^{\text {perf }}$ denote the $\infty$-category of small idempotent-complete stable $\infty$-categories. There is an idempotent completion functor given as the left adjoint to the inclusion $\mathrm{Cat}_{\infty}^{\text {perf }} \rightarrow \mathrm{Cat}_{\infty}^{\mathrm{ex}}[47,5.1 .4 .2]$, which we denote by Idem. 
Definition 2.14 Let $\mathcal{A}$ and $\mathcal{B}$ be small stable $\infty$-categories. Then we will say that $\mathcal{A}$ and $\mathcal{B}$ are Morita equivalent if $\operatorname{Idem}(\mathcal{A})$ and $\operatorname{Idem}(\mathcal{B})$ are equivalent.

We will verify shortly that this notion of Morita equivalence is compatible with the definition given in terms of spectral categories in Definition 2.7.

\subsection{Stabilization of $\infty$-categories}

Given any $\infty$-category $\mathcal{C}$ with finite limits, we can form the stabilization $\operatorname{Stab}(\mathcal{C})$ [48, Section 1.4]. The $\infty$-category $\operatorname{Stab}(\mathcal{C})$ is stable and comes equipped with a limit-preserving functor

$$
\Omega^{\infty}: \operatorname{Stab}(\mathcal{C}) \longrightarrow \mathcal{C}
$$

If in addition $\mathcal{C}$ is presentable, then $\Omega^{\infty}$ admits a left adjoint

$$
\Sigma_{+}^{\infty}: \mathcal{C} \longrightarrow \operatorname{Stab}(\mathcal{C})
$$

by $[48,1.4 .4 .4]$.

We now recall an explicit model of the stabilization of an $\infty$-category in terms of spectrum objects [48, Section 1.4.2]. Recall that a spectrum object of a pointed $\infty-$ category $\mathcal{C}$ consists of a functor $A$ from the category of finite pointed spaces to $\mathcal{C}$ that is reduced (takes the terminal object to the terminal object) and excisive (takes cocartesian squares to cartesian squares) $[48,1.4 .2 .8]$. Since the restriction of $A$ to the spheres characterizes $A$, we set $A_{i}=A\left(S^{i}\right)$ and often refer to $A$ simply by the collection of pointed objects $\left\{A_{i}\right\}$. We write $\operatorname{Sp}(\mathcal{C})$ for the $\infty$-category of spectrum objects in $\mathcal{C} ; \operatorname{Sp}(\mathcal{C})$ comes equipped with a functor $\Omega^{\infty}: \operatorname{Sp}(\mathcal{C}) \rightarrow \mathcal{C}$ which associates to the spectrum object $A$ its zero space $A_{0}=A\left(S^{0}\right)$. This is an explicit model for the stabilization $\operatorname{Stab}(\mathcal{C})$ discussed previously. To ease notation, we will usually just write $\mathcal{T}_{\infty} \simeq \mathrm{N}\left(\mathcal{T}^{\text {cf }}\right)$ for the $\infty$-category of spaces and $\mathcal{S}_{\infty} \simeq \operatorname{Sp}\left(\mathcal{T}_{\infty}\right) \simeq \mathrm{N}\left(\mathcal{S}^{\text {cf }}\right)$ for the $\infty$-category of spectra.

Now suppose that $\mathcal{C}$ is an arbitrary $\infty$-category. The Yoneda embedding $\mathcal{C} \rightarrow$ $\operatorname{Fun}\left(\mathcal{C}^{\mathrm{op}}, \mathcal{T}_{\infty}\right)$ preserves finite limits (when they exist), so it induces a functor

$$
\operatorname{Sp}\left(\mathcal{C}_{*}\right) \longrightarrow \operatorname{Sp}\left(\operatorname{Fun}\left(\mathcal{C}^{\mathrm{op}}, \mathcal{T}_{\infty}\right)_{*}\right) \simeq \operatorname{Fun}\left(\mathcal{C}^{\mathrm{op}}, \mathcal{S}_{\infty}\right)
$$

on the level of spectrum objects, where $\mathcal{C}_{*}$ denotes the category of pointed objects in $\mathcal{C}$. Here the last equivalence follows from the fact that limits in functor categories are computed pointwise, and observe also that $\mathcal{C}_{*}$ will be empty unless $\mathcal{C}$ has a final object. On the other hand, if $\mathcal{C}$ is a stable $\infty$-category, then $\mathcal{C} \simeq \mathcal{C}_{*}$ and $\Omega^{\infty}: \operatorname{Sp}(\mathcal{C}) \rightarrow \mathcal{C}$ is an equivalence with inverse $\Sigma^{\infty}: \mathcal{C} \rightarrow \operatorname{Sp}(\mathcal{C})$ given by $\left(\Sigma^{\infty} a\right)_{i}=\Sigma^{i} a$. This motivates the following definition: 
Definition 2.15 Let $\mathcal{C}$ be a stable $\infty$-category. The spectral Yoneda embedding is the composite

$$
\mathcal{C} \simeq \operatorname{Sp}\left(\mathcal{C}_{*}\right) \longrightarrow \operatorname{Sp}\left(\operatorname{Fun}\left(\mathcal{C}^{\mathrm{op}}, \mathcal{T}_{\infty}\right)_{*}\right) \simeq \operatorname{Fun}\left(\mathcal{C}^{\mathrm{op}}, \mathcal{S}_{\infty}\right)
$$

The mapping spectrum functor

$$
\text { Map: } \mathcal{C}^{\text {op }} \times \mathcal{C} \longrightarrow \mathcal{S}_{\infty}
$$

is the adjoint of the spectral Yoneda embedding.

Informally, the mapping spectrum is described by the formula

$$
\operatorname{Map}(b, a)_{i} \simeq \operatorname{map}\left(b, \Sigma^{i} a\right) .
$$

Note that this is a functor to the $\infty$-category of spectra; this is in contrast to the (point-set) mapping space functors from the category of quasicategories to the category of simplicial sets described in $[47,1.2 .2]$ or [25].

We wish to characterize the image of $\mathcal{C}$ under the spectral Yoneda embedding:

Definition 2.16 Let $\mathcal{C}$ be an $\infty$-category. We will say that a functor $X: \mathcal{C}^{\text {op }} \rightarrow \mathcal{S}_{\infty}$ is stably representable if there exists a spectrum object $A \in \operatorname{Sp}\left(\mathcal{C}_{*}\right)$ and an equivalence $\operatorname{Map}(-, A) \simeq X$, where $\operatorname{Map}(-, A)$ denotes the functor $\mathcal{C}^{\text {op }} \rightarrow \mathcal{S}_{\infty}$ represented by $A$ via the spectral Yoneda embedding $\operatorname{Sp}\left(\mathcal{C}_{*}\right) \rightarrow \operatorname{Fun}\left(\mathcal{C}^{\mathrm{op}}, \mathcal{S}_{\infty}\right)$.

When $\mathcal{C}$ is stable already, the following proposition gives an easy characterization of stably representable functors.

Proposition 2.17 Let $\mathcal{C}$ be a stable $\infty$-category. Then a functor $X: \mathcal{C}^{\mathrm{op}} \rightarrow \mathcal{S}_{\infty}$ is stably representable if and only if it is represented by the suspension spectrum $\Sigma^{\infty} z$ of a unique (up to equivalence) object $z$ of $\mathcal{C}$.

Proof It suffices to show that any spectrum object $A$ of $\mathcal{C}$ is of the form $\Sigma^{\infty} z$ for a uniquely determined object $z$ of $\mathcal{C}$. This follows from the fact that since $\mathcal{C}$ is stable, $\Omega^{\infty}: \operatorname{Sp}(\mathcal{C}) \rightarrow \mathcal{C}$ is an equivalence with inverse $\Sigma^{\infty}: \mathcal{C} \rightarrow \operatorname{Sp}(\mathcal{C})$.

\subsection{Compact objects and compactly generated $\infty$-categories}

The categorical data which serves as the input to algebraic $K$-theory is typically obtained as the objects in a larger ambient category (with weak equivalences and extension sequences) that satisfy some sort of "smallness" condition; eg the perfect complexes as a subcategory of all complexes. A key insight initially codified by Thomason-Trobaugh [72] and subsequently elaborated upon by Neeman [57] is that this example is generic 
in algebraic $K$-theory, and the typical situation involves working with the compact objects in some model of a triangulated category, which is generated under homotopy colimits by those compact objects. Thus, we will systematically regard the small stable idempotent-complete $\infty$-categories that are the domain of the algebraic $K$-theory functor as arising as the compact objects in a larger category.

This notion of looking at large categories which are in some sense determined by the compact objects is axiomatized in category theory with the formalism of accessible and locally presentable categories, introduced by Makkai and Paré [49] and further developed by Adámek and Rosický [1]. This theory was integrated into homotopy theory in Jeff Smith's theory of combinatorial model categories and developed further in this context in the seminal work of Dugger [24].

A version of this theory forms the basis for Lurie's theory of presentable $\infty$-categories, which is the analogue in the $\infty$-category setting of the homotopy theories encoded by presentable combinatorial model category structures (see also Simpson's related work in the context of Segal spaces [66]). We use this approach to handle the set-theoretic issues that arise in our work, along the lines described in [47, 1.2.15, 5.4.1]. As indicated in Remark 2.6, it is also possible to handle some of the set-theoretic technicalities that arise (ie in the context of the Yoneda Lemma) by explicit size bounds.

This framework is related to Grothendieck's universe formalization, allowing us to handle small and large $\infty$-categories on similar grounds. In particular, [47, Section 5] has extensive discussion of the interaction of the Yoneda embeddings (which arise pervasively in this context) with set-theoretic concerns. In addition to Lurie's work, the paper of Ben-Zvi, Francis and Nadler [6, Sections 2 and 4.1] provides a nice exposé of this theory in the context of the study of geometric function theory from a perspective with its origin in Thomason-Trobaugh.

Roughly speaking, presentable $\infty$-categories are large $\infty$-categories that are generated under sufficiently large filtered colimits by some small $\infty$-category. To make this precise, we need to discuss the notion of the Ind-category. Given any small $\infty-$ category $\mathcal{C}$, we can form the $\infty$-category $\operatorname{Pre}(\mathcal{C})$ of presheaves of simplicial sets on $\mathcal{C}$, which is the formal closure of $\mathcal{C}$ under colimits; that is, there is a fully faithful Yoneda embedding $\mathcal{C} \rightarrow \operatorname{Pre}(\mathcal{C})$, and $\operatorname{Pre}(\mathcal{C})$ is generated by the image of $\mathcal{C}$ under small colimits $[47,5.1 .5 .8]$. For any $\infty$-category $\mathcal{C}$ and infinite regular cardinal $\kappa$, we can form the Ind-category $\operatorname{Ind}_{\kappa}(\mathcal{C})$, which is the formal closure under $\kappa$-filtered colimits of $\mathcal{C}$ [47, Section 5.3.5]. The $\infty$-category $\operatorname{Ind}_{\kappa}(\mathcal{C})$ is a full subcategory of $\operatorname{Pre}(\mathcal{C})$, and the Yoneda embedding $\mathcal{C} \rightarrow \operatorname{Pre}(\mathcal{C})$ factors as $\mathcal{C} \rightarrow \operatorname{Ind}_{\kappa}(\mathcal{C}) \rightarrow \operatorname{Pre}(\mathcal{C})$. We record here the following useful properties of the construction of the Ind-category. 
Proposition 2.18 Let $\mathcal{C}$ be a small $\infty$-category and $\kappa$ an infinite regular cardinal.

- The $\infty$-category $\operatorname{Ind}_{\kappa}(\mathcal{C})$ admits all $\kappa$-small colimits that exist in $\mathcal{C}[47$, 5.3.5.14, 5.5.1.1].

- The functor $\mathcal{C} \rightarrow \operatorname{Ind}_{\kappa}(\mathcal{C})$ preserves $\kappa$-filtered colimits [47, 5.3.5.2, 5.3.5.3].

- $\operatorname{Ind}_{\kappa}(\mathcal{C})$ is a stable $\infty$-category [48, 1.1.3.6].

- The image of $\mathcal{C}$ in $\operatorname{Ind}_{\kappa}(\mathcal{C})$ provides a set of compact objects which generates $\operatorname{Ind}(\mathcal{C})$ under $\kappa$-filtered colimits [47, 5.3.5.5, 5.3.5.11].

- The category $\operatorname{Ind}_{\kappa}$ is characterized by the property that it has $\kappa$-small filtered colimits, admits a functor $\mathcal{C} \rightarrow \operatorname{Ind}_{\kappa}(\mathcal{C})$, and this functor induces an equivalence

$$
\operatorname{Fun}_{\kappa}\left(\operatorname{Ind}_{\kappa}(\mathcal{C}), \mathcal{D}\right) \longrightarrow \operatorname{Fun}(\mathcal{C}, \mathcal{D}),
$$

for any $\mathcal{D}$ which admits $\kappa$-filtered colimits (here $\operatorname{Fun}_{\kappa}(-,-)$ denotes the $\infty$-category of functors that preserve $\kappa$-small filtered colimits) [47, 5.3.5.10].

We now recall the following definitions [47, 5.4.2.1, 5.5.1.1].

Definition 2.19 An $\infty$-category $\mathcal{C}$ is accessible if there exists a regular cardinal $\kappa$ and a small $\infty$-category $\mathcal{C}^{0}$ such that there is an equivalence

$$
\operatorname{Ind}_{\kappa}\left(\mathcal{C}^{0}\right) \simeq \mathcal{C} .
$$

An $\infty$-category $\mathcal{C}$ is presentable if it arises as $\operatorname{Ind}_{\kappa}(\mathcal{D})$ for a small $\infty$-category $\mathcal{D}$ which admits $\kappa$-small colimits.

A morphism of presentable $\infty$-categories is a left adjoint functor; by the adjoint functor theorem [47, 5.5.2.9], a functor between presentable $\infty$-categories is a left adjoint if and only if it preserves colimits. We let $\mathcal{P r}^{\mathrm{L}}$ denote the $\infty$-category of presentable $\infty$-categories and colimit-preserving functors; the $\infty$-category of colimit-preserving functors is denoted by $\operatorname{Fun}^{\mathrm{L}}(-,-)$. In fact, $\operatorname{Fun}^{\mathrm{L}}(-,-)$ is in fact itself a presentable $\infty$-category $[47,5.5 .3 .8]$, yielding an internal hom object for $\mathcal{P r}^{\mathrm{L}}$.

We now restrict attention to the situation in which $\kappa=\omega$. Recall that an object $x$ of an $\infty$-category $\mathcal{C}$ is compact if the functor $\mathcal{C}^{\mathrm{op}} \rightarrow \mathcal{T}_{\infty}$ represented by $x$ commutes with filtered colimits [47, Section 5.3.4]. Given an $\infty$-category $\mathcal{C}$, let $\mathcal{C}^{\omega}$ denote the full subcategory of $\mathcal{C}$ consisting of the compact objects of $\mathcal{C}$. A presentable $\infty$-category $\mathcal{C}$ is compactly generated if the natural functor

$$
\operatorname{Ind}\left(\mathcal{C}^{\omega}\right) \longrightarrow \mathcal{C}
$$


which sends a filtered diagram in $\mathcal{C}^{\omega}$ to its colimit in $\mathcal{C}$, is an equivalence. There is a correspondence between small idempotent-complete $\infty$-categories and compactly generated $\infty$-categories given by the construction of the Ind-category [47, Section 5.5.7]. More generally, the construction of the Ind-category sets up a correspondence between the $\infty$-category of compactly generated presentable $\infty$-categories with morphisms colimit-preserving functors that preserve compact objects and $\mathrm{Cat}_{\infty}$; the other direction is given by passage to compact objects [47, 5.5.7.10].

The preceding discussion carries over when we restrict attention to stable categories. In this setting, the stabilization $\operatorname{Stab}(\mathcal{C})$ is initial amongst presentable stable $\infty$-categories admitting a functor from $\mathcal{C}[48,1.4 .4 .5]$, in the sense that if $\mathcal{D}$ is a presentable stable $\infty$-category then $\Sigma_{+}^{\infty}$ induces an equivalence

$$
\operatorname{Fun}^{\mathrm{L}}(\operatorname{Stab}(\mathcal{C}), \mathcal{D}) \longrightarrow \operatorname{Fun}^{\mathrm{L}}(\mathcal{C}, \mathcal{D})
$$

The $\infty$-category of stable presentable $\infty$-categories $\mathcal{P r}_{\mathrm{St}}^{\mathrm{L}}$ is a full subcategory of $\mathcal{P r}^{\mathrm{L}}$, and the Ind-category sets up a correspondence between $\mathrm{Cat}_{\infty}^{\text {perf }}$ and compactly generated stable $\infty$-categories. We may also apply Ind to nonidempotent-complete stable $\infty-$ categories to obtain a correspondence between $\mathrm{Cat}_{\infty}^{\mathrm{ex}}$ and compactly generated stable $\infty$-categories; however, these two $\infty$-categories are rather less closely related, as the full subcategory of compact objects is always idempotent-complete.

Lemma 2.20 $\mathrm{Cat}_{\infty}^{\text {perf }}$ is a reflective subcategory of $\mathrm{Cat}_{\infty}^{\mathrm{ex}}$, and the localization functor Idem: $\mathrm{Cat}_{\infty}^{\mathrm{ex}} \rightarrow \mathrm{Cat}_{\infty}^{\text {perf }}$ is given by the formula $\operatorname{Idem}(\mathcal{C}) \simeq \operatorname{Ind}(\mathcal{C})^{\omega}$.

Proof The subcategory of compact objects $\operatorname{Ind}(\mathcal{C})^{\omega}$ of $\operatorname{Ind}(\mathcal{C})$ is an idempotentcomplete stable $\infty$-category, so that Idem is indeed a functor $\mathrm{Cat}_{\infty}^{\mathrm{ex}} \rightarrow \mathrm{Cat}_{\infty}^{\text {perf }}$. Now for small stable $\infty$-categories $\mathcal{C}$ and $\mathcal{D}$ with $\mathcal{D}$ idempotent-complete, we have a commuting square

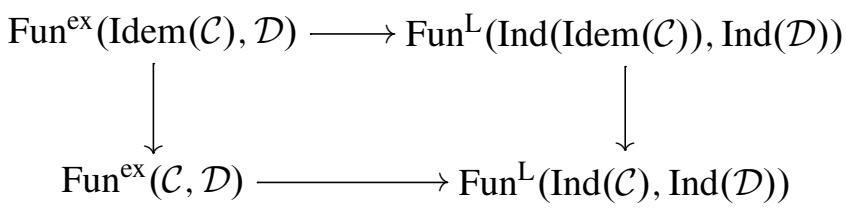

in which the horizontal maps are the inclusions of the full subcategories of functors which preserve compact objects, and the right vertical map is an equivalence as the natural map $\operatorname{Ind}(\mathcal{C}) \rightarrow \operatorname{Ind}(\operatorname{Idem}(\mathcal{C}))$ is an equivalence. Hence $\operatorname{Ind}(\mathcal{C})^{\omega} \rightarrow \operatorname{Ind}(\operatorname{Idem}(\mathcal{C}))^{\omega}$ is an equivalence, and thus the left vertical map is as well. 


\subsection{Localization of $\infty$-categories}

Given an $\infty$-category $\mathcal{C}$ and a suitable collection of morphisms $S$, one might hope to form the localization $\mathcal{C}\left[S^{-1}\right]$. This is by definition an $\infty$-category equipped with a functor $f: \mathcal{C} \rightarrow \mathcal{C}\left[S^{-1}\right]$ which satisfies the following universal property: for any other $\infty$-category $\mathcal{D}$, restriction along $f$ identifies

$$
\operatorname{Fun}\left(\mathcal{C}\left[S^{-1}\right], \mathcal{D}\right) \longrightarrow \operatorname{Fun}(\mathcal{C}, \mathcal{D})
$$

as the full subcategory of $\operatorname{Fun}(\mathcal{C}, \mathcal{D})$ spanned by those functors which send the morphisms in $S$ to equivalences in $\mathcal{D}$. If $S$ is a small set, then $\mathcal{C}\left[S^{-1}\right]$ exists in the same universe as $\mathcal{C}$; indeed, without loss of generality we may assume that $S$ contains all degenerate edges of the simplicial set $\mathcal{C}$, in which case $\mathcal{C}\left[S^{-1}\right]$ may be constructed as a fibrant replacement of $(\mathcal{C}, S)$ in the model category of marked simplicial sets.

Often in practice, however, $S$ is not small, and the existence of $\mathcal{C}\left[S^{-1}\right]$ (without passing to a higher universe) requires more delicate analysis. One standard method is to show that $\mathcal{C}$ is presentable and $S$ is (generated by) a small set of arrows in a certain sense: this is the theory of Bousfield localization, following Bousfield's seminal work on the subject [18]. In this case we may identify the localization as the full subcategory of $\mathcal{C}$ spanned by the $S$-local objects.

In model categories, there is a well-developed theory of Bousfield localization (see for example Hirschhorn's comprehensive discussion in [39], Goerss and Jardine's treatment in the simplicial setting [34], or the exposition of Smith's theory for combinatorial model categories in [3]). Because localization is a central technical device in our work, in this section we provide a brief review of Lurie's version of localization in the setting of presentable $\infty$-categories from [47, Sections 5.2.7 and 5.5.4].

Specifically, we say that a colimit preserving functor $f: \mathcal{C} \rightarrow \mathcal{D}$ of presentable $\infty-$ categories $\mathcal{C}$ and $\mathcal{D}$ is a Bousfield localization if the right adjoint of $f$ (which exists by the adjoint functor theorem) is fully faithful [47, 5.2.7.2]. When the context is clear, we tend to abuse notation and simply refer to this as a localization. A useful observation is that this data induces an equivalence between $\mathcal{D}$ and a full subcategory of $\mathcal{C}$, called the subcategory of local objects. In fact, [47, 5.2.7.4] gives a useful criterion for an endofunctor $L: \mathcal{C} \rightarrow \mathcal{C}$ to be a localization. Specifically, the following are equivalent:

(i) There exists a functor $f: \mathcal{C} \rightarrow \mathcal{D}$ with a fully faithful right adjoint $g$ and an equivalence $g \circ f \simeq L$.

(ii) When regarded as a functor $\mathcal{C} \rightarrow L \mathcal{C}, L$ is the left adjoint of the inclusion $L \mathcal{C} \rightarrow \mathcal{C}$.

(iii) There exists a natural transformation $\alpha: \operatorname{id}_{\mathcal{C}} \rightarrow L$ such that for objects $X$ in $\mathcal{C}$, the morphisms $L(\alpha(X))$ and $\alpha(L X)$ are both equivalences. 
Recall that a functor is accessible if it is $\kappa$-continuous (preserves $\kappa$-filtered colimits) for some sufficiently large regular cardinal $\kappa[47,5.4 .2 .5]$. A localization is accessible if $g$ or $L$ are accessible functors (equivalently, see [47, 5.5.1.2]) or the essential image $L \mathcal{C}$ is an accessible subcategory.

Accessible localizations of presentable categories can be completely classified as follows. Recall from [47, 5.5.4] that associated to any small set of arrows $S$ in a presentable $\infty$-category $\mathcal{C}$, the Bousfield localization $S^{-1} \mathcal{C}$ is equivalent to the ordinary localization $\mathcal{C}\left[T^{-1}\right]$ of $\mathcal{C}$ at the strongly saturated class $T$ generated by $S$ $[47,5.5 .4 .5]$. In particular, many different sets $S$ can generate the same strongly saturated class $T$; they all define the same full subcategory $S^{-1} \mathcal{C}$ of $\mathcal{C}$ of $S$-local objects [47, 5.5.4.15], where $S$-local is defined in the standard fashion [47, 5.5.4.1]. An accessible localization of a presentable $\infty$-category is presentable. As the notation suggests, Bousfield localization is characterized by the following universal property [47, 5.5.4.20]: for any other presentable $\infty$-category $\mathcal{D}$, composition with $L$ induces a functor

$$
\operatorname{Fun}^{\mathrm{L}}\left(S^{-1} \mathcal{C}, \mathcal{D}\right) \longrightarrow \operatorname{Fun}^{\mathrm{L}}(\mathcal{C}, \mathcal{D})
$$

that is fully faithful and whose essential image consists of those colimit-preserving functors which take elements of $S$ to equivalences.

\section{Symmetric monoidal structure and dualizable objects}

In this section we study the theory of dualizable objects in $\mathrm{Cat}_{\infty}^{\mathrm{perf}}$. To this end, we need to give a very brief review of the construction of the symmetric monoidal structure on $\mathrm{Cat}_{\infty}^{\text {perf }}$. We do not give a full review of the theory of monoidal $\infty$-categories in this section, since we need only a small piece of the theory.

\subsection{Tensor products of stable $\infty$-categories}

The $\infty$-category $\mathcal{P r}_{\text {St }}^{\mathrm{L}}$ of presentable stable $\infty$-categories is a closed symmetric monoidal $\infty$-category with product $\otimes$ and internal mapping object given by the presentable stable $\infty$-category $\operatorname{Fun}^{\mathrm{L}}(\mathcal{A}, \mathcal{B})$ of colimit-preserving functors [48, 6.3.1.14, 6.3.1.17]. Following [6, Section 4.1.2], we can then define the tensor product on small idempotent-complete stable $\infty$-categories as

$$
\mathcal{C} \widehat{\otimes} \mathcal{D}=(\operatorname{Ind}(\mathcal{C}) \otimes \operatorname{Ind}(\mathcal{D}))^{\omega} .
$$

The tensor product of idempotent-complete small stable $\infty$-categories is characterized by the universal property that maps out of $\mathcal{A} \otimes \mathcal{B}$ correspond to maps out of the product $\mathcal{A} \times \mathcal{B}$ which preserve finite colimits in each variable [6, 4.4]. If $\mathcal{A}$ and $\mathcal{B}$ are arbitrary small stable $\infty$-categories, then we set $\mathcal{A} \widehat{\otimes} \mathcal{B}:=\operatorname{Idem}(\mathcal{A}) \widehat{\otimes} \operatorname{Idem}(\mathcal{B})$. 
More precisely, we can define $\mathrm{Cat}_{\infty}^{\text {perf }}$ as a symmetric monoidal $\infty$-category as follows. Let $\mathcal{P} \mathrm{r}_{\mathrm{St}, \mathrm{cg}}^{\mathrm{L}}$ denote the full subcategory of $\mathcal{P r}_{\mathrm{St}}^{\mathrm{L}}$ on the compactly generated stable $\infty-$ categories. The criterion of $[48,2.2 .1 .2]$ implies that $\mathcal{P r}_{\text {St,cg }}^{\mathrm{L}}$ is a symmetric monoidal subcategory of $\mathcal{P r}_{\mathrm{St}}^{\mathrm{L}}$; the tensor product of compactly generated stable $\infty$-categories is itself compactly generated, as is the unit $\mathcal{S}_{\infty} \simeq \operatorname{Ind}\left(\mathcal{S}_{\infty}^{\omega}\right)$.

For a small idempotent-complete stable $\infty$-category $\mathcal{A}$ and a presentable $\infty$-category $\mathcal{B}$, Fun $^{\text {ex }}$ and Fun $^{\mathrm{L}}$ are related by the formula

$$
\operatorname{Fun}^{\mathrm{ex}}(\mathcal{A}, \mathcal{B}) \simeq \operatorname{Fun}^{\mathrm{L}}(\operatorname{Ind}(\mathcal{A}), \mathcal{B}),
$$

which follows from $[47,5.3 .5 .10]$ and the fact that functors which preserve filtered colimits and finite colimits preserve all colimits. Note that

$$
\text { Ind: } \mathrm{Cat}_{\infty}^{\text {perf }} \longrightarrow \mathcal{P r}_{\mathrm{St}}^{\mathrm{L}}
$$

factors through the full subcategory $\mathcal{P} \mathrm{r}_{\mathrm{St}, \mathrm{cg}}^{\mathrm{L}}$ by definition. This gives an equivalence of $\infty$-categories between Cat ${ }_{\infty}^{\text {perf }}$ and the subcategory $\mathcal{P r}_{\mathrm{St}, \omega}^{\mathrm{L}}$ of $\mathcal{P r}_{\mathrm{St}}^{\mathrm{L}}$ whose objects are the compactly generated stable $\infty$-categories and whose maps

$$
\operatorname{Fun}^{\mathrm{ex}}(\mathcal{A}, \mathcal{B}) \simeq \operatorname{Fun}_{\omega}^{\mathrm{L}}(\operatorname{Ind}(\mathcal{A}), \operatorname{Ind}(\mathcal{B})) \subset \operatorname{Fun}^{\mathrm{L}}(\operatorname{Ind}(\mathcal{A}), \operatorname{Ind}(\mathcal{B})),
$$

are the full subcategory of the colimit-preserving functors $\operatorname{Ind}(\mathcal{A}) \rightarrow \operatorname{Ind}(\mathcal{B})$ which preserve compact objects $[47,5.5 .7 .10]$. We regard $\mathrm{Cat}_{\infty}^{\text {perf }}$ as a symmetric monoidal $\infty$-category via this equivalence. The observation of $[48,6.3 .1 .17]$ implies that $\mathrm{Cat}_{\infty}^{\text {perf }}$ is closed. Hence we have the following result.

Theorem 3.1 The $\infty$-category of small idempotent-complete stable $\infty$-categories is a closed symmetric monoidal $\infty$-category with respect to $\widehat{\otimes}$. The unit is the $\infty-$ category $\mathcal{S}_{\infty}^{\omega}$ of compact spectra and the internal mapping object is given for small idempotent-complete stable $\infty$-categories $\mathcal{A}$ and $\mathcal{B}$ by $\operatorname{Fun}^{\mathrm{ex}}(\mathcal{A}, \mathcal{B})$.

Given a small idempotent-complete stable $\infty$-category $\mathcal{A}$, we have the $\infty$-category of $\mathcal{A}$-modules, given by the compactly generated stable $\infty$-category $\operatorname{Fun}^{\mathrm{ex}}\left(\mathcal{A}^{\mathrm{op}}, \mathcal{S}_{\infty}\right)$. The stable Yoneda embedding provides an exact functor [47, 5.3.5.2]

$$
\mathcal{A} \longrightarrow \operatorname{Fun}^{\mathrm{ex}}\left(\mathcal{A}^{\mathrm{op}}, \mathcal{S}_{\infty}\right)
$$

Proposition 3.2 For any small stable $\infty$-category $\mathcal{A}$, the stable Yoneda embedding

$$
\mathcal{A} \longrightarrow \operatorname{Fun}^{\mathrm{ex}}\left(\mathcal{A}^{\mathrm{op}}, \mathcal{S}_{\infty}\right)
$$

induces an equivalence $\operatorname{Ind}(\mathcal{A}) \simeq \operatorname{Fun}^{\mathrm{ex}}\left(\mathcal{A}^{\mathrm{op}}, \mathcal{S}_{\infty}\right)$. 
Proof Clearly Fun ${ }^{\mathrm{ex}}\left(\mathcal{A}^{\mathrm{op}}, \mathcal{S}_{\infty}\right)$ admits filtered colimits, as the filtered colimit of finite colimit preserving functors itself preserves finite colimits. This gives a map $\operatorname{Ind}(\mathcal{A}) \rightarrow \operatorname{Fun}^{\mathrm{ex}}\left(\mathcal{A}^{\mathrm{op}}, \mathcal{S}_{\infty}\right)$ which is evidently fully faithful, using the fact that the usual Yoneda embedding is fully faithful and that mapping spaces between representables in $\operatorname{Fun}^{\mathrm{ex}}\left(\mathcal{A}^{\mathrm{op}}, \mathcal{S}_{\infty}\right)$ are computed as the limit

$$
\lim _{n} \Omega^{n} \operatorname{map}\left(a, \Sigma^{n} b\right) \simeq \lim _{n} \operatorname{map}\left(a, \Omega^{n} \Sigma^{n} b\right) \simeq \operatorname{map}(a, b) .
$$

To show that this map is also essentially surjective, we must show that any exact functor $f: \mathcal{A}^{\text {op }} \rightarrow \mathcal{S}_{\infty}$ is ind-representable. Consider the pullback

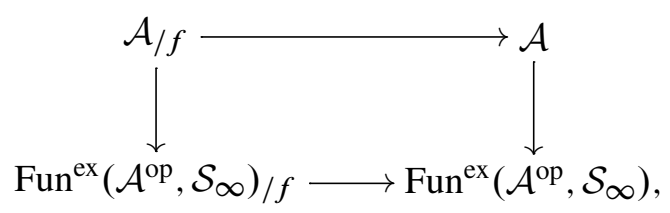

where the right vertical map is the stable Yoneda embedding. We claim that the $\infty-$ category $\mathcal{A}_{/ f}$ is filtered: to see this, let $K$ be a finite simplicial set and $K \rightarrow \mathcal{A}_{/ f}$ a functor. Since both $\mathcal{A}$ and $\operatorname{Fun}^{\mathrm{ex}}\left(\mathcal{A}^{\mathrm{op}}, \mathcal{S}_{\infty}\right) / f$ admit finite colimits and both functors to $\operatorname{Fun}^{\mathrm{ex}}\left(\mathcal{A}^{\mathrm{op}}, \mathcal{S}_{\infty}\right)$ preserve finite colimits, we may extend $K \rightarrow \mathcal{A}_{/ f}$ to a colimit diagram $K^{\triangleright} \rightarrow \mathcal{A}_{/ f}$. In particular, this gives a cone on $K \rightarrow \mathcal{A}_{/ f}$, which shows that $\mathcal{A}_{/ f}$ is a filtered $\infty$-category. Finally, since filtered colimits in $\operatorname{Fun}^{\mathrm{ex}}\left(\mathcal{A}^{\mathrm{op}}, \mathcal{S}_{\infty}\right)$ are computed pointwise, it follows that $f$ is a colimit of the diagram $\mathcal{A}_{/ f} \longrightarrow \operatorname{Fun}^{\mathrm{ex}}\left(\mathcal{A}^{\mathrm{op}}, \mathcal{S}_{\infty}\right)$, which is to say that it is ind-representable.

Provided $\mathcal{A}$ is idempotent-complete, [47, 5.4.2.4] tells us that the essential image of the Yoneda embedding is precisely the $\infty$-category of compact $\mathcal{A}$-modules

$$
\mathcal{A} \simeq \operatorname{Fun}^{\mathrm{ex}}\left(\mathcal{A}^{\mathrm{op}}, \mathcal{S}_{\infty}\right)^{\omega} .
$$

Moreover, we know that if $\mathcal{A}$ is an arbitrary small stable $\infty$-category, then the Yoneda map $\mathcal{A} \rightarrow \operatorname{Fun}^{\mathrm{ex}}\left(\mathcal{A}^{\mathrm{op}}, \mathcal{S}_{\infty}\right)^{\omega}$ models the idempotent-completion of $\mathcal{A}$.

We use the preceding results to characterize $\operatorname{Fun}^{\mathrm{ex}}(\mathcal{A}, \mathcal{B})$ in terms of a certain subcategory of $\operatorname{Fun}^{\mathrm{L}}\left(\mathcal{A} \widehat{\otimes} \mathcal{B}^{\mathrm{op}}, \mathcal{S}_{\infty}\right)$, the $\infty$-category of $\mathcal{A}-\mathcal{B}$-bimodules. Specifically, the Yoneda embedding $\mathcal{B} \rightarrow \operatorname{Fun}^{\text {ex }}\left(\mathcal{B}^{\text {op }}, \mathcal{S}_{\infty}\right)$ provides the following composite:

$$
\begin{aligned}
\operatorname{Fun}^{\mathrm{ex}}(\mathcal{A}, \mathcal{B}) & \longrightarrow \operatorname{Fun}^{\mathrm{ex}}\left(\mathcal{A}, \operatorname{Fun}^{\mathrm{ex}}\left(\mathcal{B}^{\mathrm{op}}, \mathcal{S}_{\infty}\right)\right) \\
& \longrightarrow \operatorname{Fun}^{\mathrm{L}}\left(\operatorname{Ind}(\mathcal{A}), \operatorname{Fun}^{\mathrm{L}}\left(\operatorname{Ind}\left(\mathcal{B}^{\mathrm{op}}\right), \mathcal{S}_{\infty}\right)\right) \\
& \longrightarrow \operatorname{Fun}^{\mathrm{L}}\left(\operatorname{Ind}(\mathcal{A}) \otimes \operatorname{Ind}\left(\mathcal{B}^{\mathrm{op}}\right), \mathcal{S}_{\infty}\right) \\
& \longrightarrow \operatorname{Fun}^{\mathrm{ex}}\left(\mathcal{A} \widehat{\otimes} \mathcal{B}^{\mathrm{op}}, \mathcal{S}_{\infty}\right),
\end{aligned}
$$

which exhibits $\operatorname{Fun}^{\mathrm{ex}}(\mathcal{A}, \mathcal{B})$ as a full subcategory of $\mathcal{A}^{\mathrm{op}} \widehat{\otimes} \mathcal{B}$-modules. 
We have the following useful corollary, which is the analogue of a characterization originally written down by Toën [73]. For each object $a \in \mathcal{A}$, we have a map of small idempotent-complete stable $\infty$-categories $\mathcal{S}_{\infty}^{\omega} \rightarrow \mathcal{A}$ given by sending $\mathbb{S} \in \mathcal{S}_{\infty}^{\omega}$ to $a \in \mathcal{A}$. Since $\mathcal{S}_{\infty}^{\omega}$ is the unit of the tensor $\widehat{\otimes}$, we obtain a restriction map

$$
v_{a}: \operatorname{Fun}^{\mathrm{ex}}\left(\mathcal{A} \widehat{\otimes} \mathcal{B}^{\mathrm{op}}, \mathcal{S}_{\infty}\right) \longrightarrow \operatorname{Fun}^{\mathrm{ex}}\left(\mathcal{B}^{\mathrm{op}}, \mathcal{S}_{\infty}\right) \text {. }
$$

If the image of an element of $\operatorname{Fun}^{\mathrm{ex}}\left(\mathcal{A} \widehat{\otimes} \mathcal{B}^{\mathrm{op}}, \mathcal{S}_{\infty}\right)$ under $v_{a}$ is compact for every $a \in \mathcal{A}$, we will say that the element is right-compact.

Corollary 3.3 Let $\mathcal{A}$ and $\mathcal{B}$ be small idempotent-complete stable $\infty$-categories. There is an equivalence of small idempotent-complete stable $\infty$-categories between $\operatorname{Fun}^{\mathrm{ex}}(\mathcal{A}, \mathcal{B})$ and $\infty$-category of right-compact $\mathcal{A}^{\mathrm{op}} \widehat{\otimes} \mathcal{B}$-modules.

Proof Since the Yoneda embedding is fully faithful, it suffices to look at the essential image of the composite. The image of $\operatorname{Fun}^{\mathrm{ex}}(\mathcal{A}, \mathcal{B})$ in $\operatorname{Fun}^{\mathrm{ex}}\left(\mathcal{A} \widehat{\otimes} \mathcal{B}^{\mathrm{op}}, \mathcal{S}_{\infty}\right)$ under $v_{a}$ is identified with the image of $\operatorname{Fun}^{\mathrm{ex}}(\mathcal{A}, \mathcal{B})$ in $\operatorname{Fun}^{\mathrm{ex}}\left(\mathcal{A}, \operatorname{Fun}^{\mathrm{ex}}\left(\mathcal{B}^{\mathrm{op}}, \mathcal{S}_{\infty}\right)\right)$ under the corresponding map $\mathcal{S}_{\infty}^{\omega} \rightarrow \mathcal{A}$. Since this lies inside the image of $B$ inside $\operatorname{Fun}^{\text {ex }}\left(\mathcal{B}^{\text {op }}, \mathcal{S}_{\infty}\right)$ under the Yoneda embedding, the result follows.

\subsection{Smooth and proper stable $\infty$-categories}

Our final goal in this section is to characterize the dualizable objects of Cat ${ }_{\infty}^{\text {perf }}$. To do so, we need to introduce certain smallness conditions on small stable $\infty$-categories.

Definition 3.4 A small stable $\infty$-category $\mathcal{A}$ is proper if, for all pairs of objects $a$ and $b$ of $\mathcal{A}$, the mapping spectrum $\mathcal{A}(a, b)$ (recall Definition 2.15) is compact.

Note that a small stable $\infty$-category $\mathcal{A}$ is proper if and only if its idempotentcompletion $\operatorname{Idem}(\mathcal{A})$ is proper, as retracts of compact objects are compact.

Definition 3.5 A small stable $\infty$-category $\mathcal{A}$ is smooth if it is perfect as an $\mathcal{A}^{\mathrm{op}} \widehat{\otimes} \mathcal{A}-$ module (ie in the smallest subcategory of $\operatorname{Fun}^{\mathrm{ex}}\left(\mathcal{A} \widehat{\otimes} \mathcal{A}^{\mathrm{op}}, \mathcal{S}_{\infty}\right)$ generated by the representables under finite colimits and retracts). If $\mathcal{A}$ is idempotent-complete, we may equivalently require that $\mathcal{A}$ is a representable $\mathcal{A}^{\mathrm{op}} \widehat{\otimes} \mathcal{A}$-module: since $\mathcal{A}^{\mathrm{op}} \widehat{\otimes} \mathcal{A}$ is an idempotent-complete stable $\infty$-category, it is closed under finite colimits and retracts, and so any perfect $\mathcal{A}^{\mathrm{op}} \widehat{\otimes} \mathcal{A}$-module is representable.

We will typically only be interested in smoothness and properness of small stable $\infty-$ categories which are also idempotent-complete. This is because these are the situations which arise in algebra and geometry, eg when $\mathcal{A}$ is the stable $\infty$-category of perfect 
modules for a ring spectrum or perfect complexes for a scheme, and such categories are always idempotent complete. Conversely (as we will show in Section 4) any idempotentcomplete small stable $\infty$-category $\mathcal{A}$ is equivalent to the stable $\infty$-category of perfect modules for some spectral category.

\subsection{Dualizability}

We now recall the definitions of dualizability in symmetric monoidal $\infty$-categories from [48, Section 4.2.5]. The salient fact here is that dualizability can be detected in the (symmetric monoidal) homotopy category:

Definition 3.6 Let $\mathcal{C}^{\otimes}$ be a symmetric monoidal $\infty$-category. An object of the underlying $\infty$-category $\mathcal{C}$ of $\mathcal{C}^{\otimes}$ is said to be dualizable if it is dualizable as an object of the symmetric monoidal homotopy category of $\mathcal{C}^{\otimes}$.

In other words, an object $A$ of $\mathcal{C}$ is dualizable if there exists an object $D A$ together with an evaluation map $\epsilon: A \otimes D A \rightarrow 1$ and a coevaluation map $\delta: 1 \rightarrow D A \otimes A$ such that the composites

$$
A \simeq A \otimes 1 \stackrel{A \otimes \delta}{\longrightarrow} A \otimes D A \otimes A \stackrel{\epsilon \otimes A}{\longrightarrow} 1 \otimes A \simeq A
$$

and

$$
D A \simeq 1 \otimes D A \stackrel{\delta \otimes D A}{\longrightarrow} D A \otimes A \otimes D A \stackrel{D A \otimes \epsilon}{\longrightarrow} D A \otimes 1 \simeq D A
$$

are the respective identities in $\operatorname{Ho}(\mathcal{C})$. The object $D A$ is called the dual of $A$, and is unique up to equivalence in $\mathcal{C}$.

Recall that the discussion preceding Theorem 3.1 above identifies $\mathrm{Cat}_{\infty}^{\text {perf }}$ as a symmetric monoidal subcategory of $\mathcal{P r}_{\mathrm{St}, \mathrm{Lg}}^{\mathrm{L}}$; in particular, the functor Ind preserves dualizable objects. Next, observe that $\mathcal{P} \mathrm{r}_{\mathrm{St}, \mathrm{Lg}}^{\mathrm{L}}$ is a rigid symmetric monoidal category; that is, all objects in $\mathcal{P r}_{\mathrm{St}, \mathrm{Lg}}^{\mathrm{L}}$ are dualizable. This is because, for $\mathcal{A} \in \mathrm{Cat}_{\infty}^{\text {perf }}$,

$$
\operatorname{Ind}\left(\mathcal{A}^{\mathrm{op}}\right) \simeq \operatorname{Fun}^{\mathrm{ex}}\left(\mathcal{A}, \mathcal{S}_{\infty}\right) \simeq \operatorname{Fun}^{\mathrm{L}}\left(\operatorname{Ind}(\mathcal{A}), \mathcal{S}_{\infty}\right)
$$

is the dual of $\mathcal{A}$ and the coevaluation map

$$
\mathcal{S}_{\infty} \longrightarrow \operatorname{Ind}\left(\mathcal{A}^{\mathrm{op}}\right) \otimes \operatorname{Ind}(\mathcal{A}) \simeq \operatorname{Ind}\left(\mathcal{A}^{\mathrm{op}} \widehat{\otimes} \mathcal{A}\right) \simeq \operatorname{Fun}^{\mathrm{ex}}\left(\mathcal{A} \widehat{\otimes} \mathcal{A}^{\mathrm{op}}, \mathcal{S}_{\infty}\right)
$$

is given by formation of mapping spectra in $\mathcal{A}^{\mathrm{op}}$.

In analogy with the situation for dg-categories [21, Section 4], this allow us to obtain the following characterization of the dualizable objects. 
Theorem 3.7 An idempotent-complete small stable $\infty$-category $\mathcal{A}$ is dualizable (as an object of the symmetric monoidal $\infty$-category $\mathrm{Cat}_{\infty}^{\mathrm{perf}}$ of idempotent-complete small stable $\infty$-categories) if and only if $\mathcal{A}$ is smooth and proper. Moreover, the dual of a dualizable object $\mathcal{A}$ is its opposite $\infty$-category $\mathcal{A}^{\mathrm{op}}$.

Proof By the proceeding discussion, $\operatorname{Ind}(\mathcal{A})$ is a dualizable object of $\mathcal{P r}_{\mathrm{St}, \mathrm{cg}}^{\mathrm{L}}$ with dual $\operatorname{Ind}\left(\mathcal{A}^{\mathrm{op}}\right)$. Thus the dual of $\mathcal{A}$ in $\mathrm{Cat}_{\infty}^{\mathrm{perf}}$ is $\mathcal{A}^{\mathrm{op}}$, and $\mathcal{A}$ is dualizable in $\mathrm{Cat}_{\infty}^{\mathrm{perf}} \simeq \mathcal{P r}_{\mathrm{St} \omega}^{\mathrm{L}}$ if and only if the evaluation and coevaluation maps lie in the subcategory $\mathcal{P r}_{\text {St } \omega}^{\mathrm{L}} \subset$ $\mathcal{P r}_{\mathrm{St}, \mathrm{cg}}^{\mathrm{L}}$. But the evaluation map

$$
\operatorname{Ind}\left(\mathcal{A} \widehat{\otimes} \mathcal{A}^{\mathrm{op}}\right) \simeq \operatorname{Ind}(\mathcal{A}) \otimes \operatorname{Ind}(\mathcal{A})^{*} \longrightarrow \operatorname{Ind}\left(\mathcal{S}_{\infty}^{\omega}\right) \simeq \mathcal{S}_{\infty}
$$

is induced by the mapping spectrum functor $\operatorname{Map}_{\mathcal{A}}: \mathcal{A}^{\mathrm{op}} \widehat{\otimes} \mathcal{A} \longrightarrow \mathcal{S}_{\infty}$ in $\mathcal{A}$; dually, the coevaluation map

$$
\mathcal{S}_{\infty} \simeq \operatorname{Ind}\left(\mathcal{S}_{\infty}^{\omega}\right) \longrightarrow \operatorname{Ind}\left(\mathcal{A}^{\mathrm{op}}\right) \otimes \operatorname{Ind}(\mathcal{A}) \simeq \operatorname{Ind}\left(\mathcal{A}^{\mathrm{op}} \widehat{\otimes} \mathcal{A}\right)
$$

given by the map $\mathcal{S}_{\infty} \longrightarrow \operatorname{Ind}\left(\mathcal{A}^{\text {op }} \widehat{\otimes} \mathcal{A}\right)$ which classifies $\mathcal{A}$ as an $\mathcal{A}^{\text {op }} \widehat{\otimes} \mathcal{A}$-module. Hence the evaluation map lies in this subcategory if and only if the mapping spectra $\mathcal{A}(a, b)$ in $\mathcal{A}$ are compact, and the coevaulation map lies in this subcategory if and only if $\mathcal{A}$ is a compact $\mathcal{A}^{\mathrm{op}} \widehat{\otimes} \mathcal{A}$-module. Therefore, by definition, $\mathcal{A}$ is a dualizable object of $\mathrm{Cat}_{\infty}^{\text {perf }}$ if and only if $\mathcal{A}$ is smooth and proper.

\section{Morita theory}

There is a close connection between stable $\infty$-categories and spectral categories. On the one hand, for every pair of objects in a stable $\infty$-category we can extract a mapping spectrum, as we discussed in Definition 2.15. On the other hand, given a category $\mathcal{A}$ enriched in spectra, the category of (right) $\mathcal{A}$-modules has a standard projective model structure and the associated $\infty$-category is stable.

The purpose of this section is to provide a precise account of the relationship between small spectral categories and small stable $\infty$-categories. The moral of the story is that the homotopy theory of small spectral categories, localized at the Morita equivalences, is the same as the homotopy theory of small idempotent-complete stable $\infty$-categories. Specifically, we prove Theorem 1.10 from the introduction, which can be thought of as a generalization of the Morita theory of [65]; that is, small idempotent-complete stable $\infty$-categories are $\infty$-categories of modules, and the $\infty$-category of exact functors between two such is a stable subcategory of the $\infty$-category of bimodules.

Establishing this correspondence serves several purposes for us. For one thing, having models of $\mathrm{Cat}_{\infty}^{\mathrm{ex}}$ and $\mathrm{Cat}_{\infty}^{\mathrm{perf}}$ as accessible localizations of an $\infty$-category which arises 
as the nerve of a model category provides technical control on $\mathrm{Cat}_{\infty}^{\mathrm{ex}}$ and $\mathrm{Cat}_{\infty}^{\text {perf }}$; we use this to show that $\mathrm{Cat}_{\infty}^{\mathrm{ex}}$ and $\mathrm{Cat}_{\infty}^{\text {perf }}$ are compactly generated in Corollary 4.25. For another, it permits us to rectify diagrams of small stable $\infty$-categories to strict diagrams in $\mathrm{Cat}_{\mathcal{S}}$. We exploit this to pass to rigid models for the purposes of using Waldhausen's $K$-theory machinery in Section 7.

As described in Section 2.2, we have several equivalent options for producing a model of the $\infty$-category of spectral categories (with respect to the DK-equivalences): we can use the combinatorial simplicial model structure of Corollary 2.4 and take $\mathrm{N}\left(\left(\mathrm{Cat}_{\mathcal{S}}\right)^{\mathrm{cf}}\right)$, we can use the Dwyer-Kan simplicial localization followed by fibrant replacement to obtain $\mathrm{N}\left(\left(L^{H} \mathrm{Cat}_{\mathcal{S}}\right)^{\mathrm{fib}}\right)$, or we can invert the weak equivalences and form $\mathrm{N}\left(\left(\mathrm{Cat}_{\mathcal{S}}\right)^{\mathrm{c}}\right)\left[W^{-1}\right]$. We will refer interchangeably to the underlying $\infty$-category as "the" $\infty$-category of small spectral categories.

\subsection{Stable envelopes of spectral categories}

Given any spectral category $\mathcal{C}$, we can produce an $\infty$-category by passing to the associated simplicial category, fibrantly replacing, and applying the simplicial nerve to obtain $\mathrm{N}\left(\Omega^{\infty}(\mathcal{C})^{\mathrm{fib}}\right)$. This process yields a functor

$$
\mathrm{Cat}_{\mathcal{S}} \longrightarrow \mathrm{Set}_{\Delta}
$$

from the category $\mathrm{Cat}_{\mathcal{S}}$ of small spectral categories to the category of simplicial sets. Precomposing with the functors $\widehat{(-)}_{\text {perf }}$ and $\widehat{(-)}_{\text {tri }}$, we obtain functors

$$
\psi_{\text {tri }}, \psi_{\text {perf }}: \text { Cat }_{\mathcal{S}} \longrightarrow \text { Set }_{\Delta}
$$

and a natural transformation $\psi_{\text {tri }} \rightarrow \psi_{\text {perf }}$. First, we observe that these functors are compatible with the weak equivalences of Theorem 2.2.

Lemma 4.1 Let $\mathcal{A}$ and $\mathcal{B}$ be small spectral categories, and let $f: \mathcal{A} \rightarrow \mathcal{B}$ be a $D K-$ equivalence. Then the induced maps $\psi_{\text {tri }}(f)$ and $\psi_{\text {perf }}(f)$ are categorical equivalences of simplicial sets.

Proof If $f: \mathcal{A} \rightarrow \mathcal{B}$ is a DK-equivalence, then one can check that $\left(f_{!}, f^{*}\right)$ gives a Quillen equivalence between the spectral model categories $\widehat{\mathcal{A}}$ of $\mathcal{A}$-modules and the spectral model category $\widehat{\mathcal{B}}$ of $\mathcal{B}$-modules. Passing to underlying simplicial categories of cofibrant and fibrant objects, we see that $\Omega^{\infty}(\widehat{\mathcal{A}})^{\mathrm{cf}}=\operatorname{Mod}(\mathcal{A})^{\mathrm{cf}}$ and $\Omega^{\infty}(\widehat{\mathcal{B}})=\operatorname{Mod}(\mathcal{B})^{\mathrm{cf}}$ are DK-equivalent simplicial categories. Finally, applying the simplicial nerve yields categorically equivalent simplicial sets. Restricting to various full subcategories yields the result for $\psi_{\text {tri }}(f)$ and $\psi_{\text {perf }}(f)$. 
Therefore, we have induced functors $\Psi_{\text {tri }}$ and $\Psi_{\text {perf }}$ connecting $\mathrm{N}\left(\left(\mathrm{Cat}_{\mathcal{S}}\right)^{\mathrm{c}}\right)\left[W^{-1}\right]$ and $\mathrm{N}\left(\left(\operatorname{Set}_{\Delta}\right)^{\mathrm{c}}\right)\left[W^{-1}\right]$, equipped with a natural transformation connecting them:

$$
\Psi_{\text {tri }} \longrightarrow \Psi_{\text {perf }}: \mathrm{N}\left(\left(\mathrm{Cat}_{\mathcal{S}}\right)^{\mathrm{c}}\right)\left[W^{-1}\right] \longrightarrow \mathrm{N}\left(\left(\operatorname{Set}_{\Delta}\right)^{\mathrm{c}}\right)\left[W^{-1}\right] \simeq \mathrm{Cat}_{\infty} .
$$

In fact, by construction these functors preserve triangulated and Morita equivalences respectively. Furthermore, $\Psi_{\text {tri }}$ lands in small stable $\infty$-categories and $\Psi_{\text {perf }}$ lands in idempotent-complete stable $\infty$-categories.

Lemma 4.2 $\Psi_{\text {tri }} \mathcal{C}$ factors through the subcategory $\mathrm{Cat}_{\infty}^{\mathrm{ex}} \subset \mathrm{Cat}_{\infty}$, and $\Psi_{\text {perf }} \mathcal{C}$ factors through the subcategory $\mathrm{Cat}_{\infty}^{\text {perf }} \subset \mathrm{Cat}_{\infty}$.

Proof As noted in Remark 2.13, since $\psi_{\text {tri }}$ is the underlying simplicial category associated to a pretriangulated spectral category, $\Psi_{\text {perf }}$ is characterized as the idempotentcompletion of $\psi_{\text {tri }}$ given by Proposition 3.2 coupled with Corollary 3.3. Finally, note that maps of spectral categories induce, by left Kan extension, finite colimit-preserving on the level of stable $\infty$-categories.

Consequently, we may regard $\Psi_{\text {tri }}$ as a functor $\mathrm{N}\left(\left(\mathrm{Cat}_{\mathcal{S}}\right)^{\mathrm{c}}\right)\left[W^{-1}\right] \rightarrow \mathrm{Cat}_{\infty}^{\mathrm{ex}}$ and $\Psi_{\text {perf }}$ as a functor $\mathrm{N}\left(\left(\mathrm{Cat}_{\mathcal{S}}\right)^{\mathrm{c}}\right)\left[W^{-1}\right] \rightarrow \mathrm{Cat}_{\infty}^{\text {perf }}$.

Remark 4.3 Using the machinery of combinatorial simplicial model categories, we can also localize the combinatorial model structure of Corollary 2.4 on Cat $\mathcal{S}$ at the triangulated or Morita equivalences directly to obtain "triangulated" or "Morita" simplicial model categories on small spectral categories and then pass to simplicial nerves; this is equivalent to localizing the $\infty$-category $\mathrm{N}\left(\left(\mathrm{Cat}_{\mathcal{S}}\right)^{\mathrm{cf}}\right)$.

The content of Theorem 1.10 is that these functors are equivalences. We prove this theorem by producing an "inverse" to $\Psi_{\text {tri }}$ and $\Psi_{\text {perf }}$ such that the composite is a localization functor on $\mathrm{N}\left(\left(\mathrm{Cat}_{\mathcal{S}}\right)^{\mathrm{c}}\right)\left[W^{-1}\right]$. We begin with the following definition:

Definition 4.4 A simplicial category $\mathcal{A}$ is stable if the simplicial nerve of a fibrant replacement of $\mathcal{A}$ is a stable $\infty$-category. A spectral category $\mathcal{A}$ is stable if its underlying simplicial category $\Omega^{\infty} \mathcal{A}$ is stable.

We have the following characterization of equivalences between stable spectral categories (see also [10, 5.7]).

Proposition 4.5 Let $\mathcal{A}$ and $\mathcal{B}$ be stable spectral categories. Then a spectral functor $f: \mathcal{A} \rightarrow \mathcal{B}$ is a $D K$-equivalence if and only if

$$
\operatorname{Ho}\left(\Omega^{\infty} f\right): \operatorname{Ho}\left(\Omega^{\infty} \mathcal{A}\right) \longrightarrow \operatorname{Ho}\left(\Omega^{\infty} \mathcal{B}\right)
$$

is an equivalence. 
Proof Certainly essential surjectivity is determined on the level of the homotopy category, so it suffices to show that, for all pairs of objects $a$ and $b$ of $\mathcal{A}, \pi_{n} \operatorname{Map}(a, b) \rightarrow$ $\pi_{n} \operatorname{Map}(f a, f b)$ for all integers $n$ whenever this is the case for $n=0$. Since $\mathcal{A}$ and $\mathcal{B}$ are stable,

$$
\pi_{0} \operatorname{Map}\left(\Sigma^{n} a, b\right) \cong \pi_{n} \operatorname{Map}(a, b) \longrightarrow \pi_{n} \operatorname{Map}(f a, f b) \cong \pi_{0} \operatorname{Map}\left(\Sigma^{n} f a, f b\right),
$$

so this is immediate.

We write $\mathrm{Cat}_{\mathcal{T}}^{\mathrm{ex}}$ for the simplicial category of small stable simplicial categories. We can model this as the subcategory of the simplicial category $L^{H}\left(\mathrm{Cat}_{\mathcal{T}}\right)$ of small simplicial categories where the objects are the stable simplicial categories and the mapping spaces are computed by restriction of vertices to those simplicial functors which represent exact functors upon passage to the simplicial nerve.

Proposition 4.6 The $\infty$-category obtained by applying the simplicial nerve to (a fibrant replacement of) $\mathrm{Cat}_{\mathcal{T}}^{\mathrm{ex}}$ is equivalent to the $\infty$-category $\mathrm{Cat}_{\infty}^{\mathrm{ex}}$. That is, the equivalence (induced by the simplicial nerve [47, 2.2.0.1])

$$
\mathrm{N}\left(\left(L^{H}\left(\mathrm{Cat}_{\mathcal{T}}\right)^{\mathrm{fib}}\right) \longrightarrow \mathrm{Cat}_{\infty}\right.
$$

restricts to an equivalence

$$
\mathrm{N}\left(\left(\mathrm{Cat}_{\mathcal{T}}^{\mathrm{ex}}\right)^{\mathrm{fib}}\right) \longrightarrow \mathrm{Cat}_{\infty}^{\mathrm{ex}}
$$

Proof It suffices to show that the mapping spaces in $\mathrm{Cat}_{\mathcal{T}}^{\mathrm{ex}}$ have the correct homotopy type, and this follows from the comparison between the mapping spaces of Cat $\mathcal{T}$ and $\mathrm{Cat}_{\infty}[47,2.2 .0 .1]$ and the fact that on both sides we define the mapping spaces by the same restriction of vertices.

\subsection{Spectral enrichment of stable $\infty$-categories}

The mapping spaces of a stable $\infty$-category $\mathcal{C}$ are naturally the underlying spaces of mapping spectra, as discussed in Section 2.3. We now use this to construct a cofibrant and fibrant spectral category $\Upsilon(\mathcal{C})$ whose underlying $\infty$-category $\mathrm{N}\left(\Omega^{\infty} \Upsilon(\mathcal{C})\right)$ is equivalent to $\mathcal{C}$. Indeed, the simplicial category of presheaves of spectra

$$
\operatorname{Fun}_{\Delta}\left(\mathfrak{C}[\mathcal{C}]^{\text {op }}, \mathcal{S}\right)
$$

on the associated (cofibrant) simplicial category $\mathfrak{C}[\mathcal{C}]$ is simultaneously a simplicial model category as well as a spectral category, where the spectral enrichment is inherited from the spectral structure on $\mathcal{S}$ itself. Moreover, we have an equivalence

$$
\mathrm{N}\left(\operatorname{Fun}_{\Delta}\left(\mathfrak{C}[\mathcal{C}]^{\text {op }}, \mathcal{S}\right)^{\mathrm{cf}}\right) \simeq \operatorname{Fun}\left(\mathcal{C}^{\mathrm{op}}, \mathcal{S}_{\infty}\right)
$$


so it makes sense to ask whether or not a given presheaf of spectra is stably representable (in the underlying $\infty$-category $\operatorname{Fun}\left(\mathcal{C}^{\mathrm{op}}, \mathcal{S}_{\infty}\right)$ ).

\section{Definition 4.7 Let}

$$
\Upsilon(\mathcal{C}) \subset \operatorname{Fun}_{\Delta}\left(\mathfrak{C}[\mathcal{C}]^{\text {op }}, \mathcal{S}\right)
$$

denote the full spectral subcategory spanned by those (projectively) cofibrant and fibrant functors which are stably representable.

Proposition 4.8 For a small stable $\infty$-category $\mathcal{C}$, there is a natural equivalence of $\infty$-categories $\mathcal{C} \rightarrow \mathrm{N}\left(\Omega^{\infty} \Upsilon(\mathcal{C})\right)$.

Proof The spectral Yoneda embedding

$$
\mathcal{C} \rightarrow \operatorname{Fun}\left(\mathcal{C}^{\mathrm{op}}, \mathcal{S}_{\infty}\right) \simeq \mathrm{N}\left(\operatorname{Fun}_{\Delta}\left(\mathfrak{C}[\mathcal{C}]^{\text {op }}, \mathcal{S}\right)^{\mathrm{cf}}\right)
$$

is adjoint to a simplicial functor

$$
\mathfrak{C}[\mathcal{C}] \longrightarrow \operatorname{Fun}_{\Delta}\left(\mathfrak{C}[\mathcal{C}]^{\mathrm{op}}, \mathcal{S}\right)^{\mathrm{cf}}
$$

which evidently factors through the full simplicial subcategory $\Omega^{\infty} \Upsilon(\mathcal{C})$ spanned by the stably representable functors. The map $\mathcal{C} \rightarrow \mathrm{N}\left(\Omega^{\infty} \Upsilon(\mathcal{C})\right)$ is the adjoint of the resulting map $\mathfrak{C}[\mathcal{C}] \rightarrow \Omega^{\infty} \Upsilon(\mathcal{C})$.

To see that this map is an equivalence, we observe first that it is essentially surjective: Indeed, a stably representable cofibrant and fibrant functor $X: \mathfrak{C}[\mathcal{C}]^{\text {op }} \rightarrow \mathcal{S}$ is necessarily of the form $X \simeq \operatorname{Map}(-, A)$ for some spectrum object $A=\left\{a_{i}\right\}$ of $\mathcal{C} \simeq \mathrm{N}\left(\mathfrak{C}[\mathcal{C}]^{\mathrm{ib}}\right)$. Since $\mathcal{C}$ is stable, $a_{i} \simeq \Sigma^{i} a$ for some object $a$ of $\mathcal{C}$, so $X$ is in the image of $\mathcal{C}$ (which sends $a$ to the presheaf represented by $\Sigma^{\infty} a$ ). This map is also fully faithful, because if $a$ and $b$ are any pair of objects of $\mathcal{C}$, then

$$
\operatorname{map}\left(\Sigma^{\infty} b, \Sigma^{\infty} a\right) \simeq \operatorname{map}\left(b, \Omega^{\infty} \Sigma^{\infty} a\right) \simeq \operatorname{map}(b, a),
$$

since $a \simeq \Omega^{\infty} \Sigma^{\infty} a$.

We have the following description of $\Upsilon$ in terms of the stable Yoneda embedding.

Proposition 4.9 Let $\mathcal{C}$ be a small stable $\infty$-category. The fully faithful inclusion

$$
\Upsilon(\mathcal{C}) \longrightarrow \operatorname{Fun}_{\Delta}\left(\mathfrak{C}[\mathcal{C}]^{\text {op }}, \mathcal{S}\right)
$$

factors, on the level of underlying $\infty$-categories, as the composite

$$
\mathrm{N}\left(\Omega^{\infty} \Upsilon(\mathcal{C})\right) \simeq \mathcal{C} \rightarrow \operatorname{Fun}^{\mathrm{ex}}\left(\mathcal{C}^{\mathrm{op}}, \mathcal{S}_{\infty}\right)^{\omega} \subseteq \operatorname{Fun}\left(\mathcal{C}^{\mathrm{op}}, \mathcal{S}_{\infty}\right) \simeq \mathrm{N}\left(\operatorname{Fun}_{\Delta}\left(\mathfrak{C}[\mathcal{C}]^{\mathrm{op}}, \mathcal{S}\right)^{\mathrm{cf}}\right)
$$


Proof Any stably representable functor $\mathcal{C}^{\mathrm{op}} \rightarrow \mathcal{S}_{\infty}$ is exact, giving the factorization

$$
\mathcal{C} \longrightarrow \operatorname{Fun}^{\mathrm{ex}}\left(\mathcal{C}^{\mathrm{op}}, \mathcal{S}_{\infty}\right) \subseteq \operatorname{Fun}\left(\mathcal{C}^{\mathrm{op}}, \mathcal{S}_{\infty}\right) .
$$

By Proposition 3.2, we may rewrite this as $\mathcal{C} \rightarrow \operatorname{Ind}(\mathcal{C}) \simeq \operatorname{Fun}^{\mathrm{ex}}\left(\mathcal{C}^{\mathrm{op}}, \mathcal{S}_{\infty}\right)$ to see that, as an exact functor $\mathcal{C}^{\text {op }} \rightarrow \mathcal{S}_{\infty}$, any stably representable functor is also compact.

Our model of Cat $\mathrm{e}_{\mathcal{T}}^{\mathrm{ex}}$ allows us to check that the construction of $\Upsilon$ induces a simplicial functor:

Proposition 4.10 The assignment which associates to the stable simplicial category $\mathcal{C}$ the spectral category $\Upsilon(\mathcal{C})$ defines a simplicial functor

$$
\Upsilon: \mathrm{Cat}_{\mathcal{T}}^{\mathrm{ex}} \longrightarrow L^{H}\left(\mathrm{Cat}_{\mathcal{S}}\right)
$$

and hence a functor of $\infty$-categories

$$
\mathrm{N}(\Upsilon): \mathrm{Cat}_{\infty}^{\mathrm{ex}} \longrightarrow \mathrm{N}\left(\left(L^{H}\left(\mathrm{Cat}_{\mathcal{S}}\right)\right)^{\mathrm{fib}}\right)
$$

Proof We first check that the construction of $\Upsilon$ induces a functor $\mathrm{Cat}_{\mathcal{T}}^{\mathrm{ex}} \rightarrow \mathrm{Cat}_{\mathcal{S}}$. Let $f: \mathcal{C} \rightarrow \mathcal{D}$ be a map of stable simplicial categories and write

$$
f_{!}^{\mathrm{cf}}: \operatorname{Fun}_{\Delta}\left(\mathcal{C}^{\mathrm{op}}, \mathcal{S}\right)^{\mathrm{cf}} \longrightarrow \operatorname{Fun}_{\Delta}\left(\mathcal{D}^{\mathrm{op}}, \mathcal{S}\right)^{\mathrm{cf}}
$$

for the induced spectral functor. Suppose that $X: \mathcal{C}^{\mathrm{op}} \rightarrow \mathcal{S}$ is projectively cofibrant and fibrant and that $\mathrm{N}(X): \mathrm{N}(\mathcal{C})^{\mathrm{op}} \rightarrow \mathcal{S}_{\infty}$ is stably representable via the spectrum object $A=\left\{a_{i}\right\}$ in NC. Since the diagram

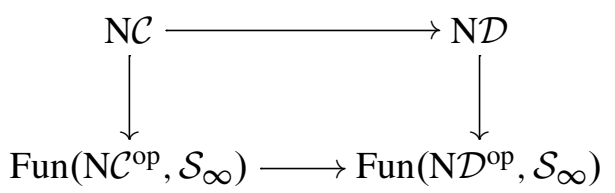

commutes (where the vertical maps are the stable Yoneda embeddings), we see that $f$ ! restricts to a spectral functor $\Upsilon(\mathcal{C}) \rightarrow \Upsilon(\mathcal{D})$.

To verify that $\Upsilon$ induces a simplicial functor, we must check that it preserves equivalences of stable simplicial categories. So suppose that $f: \mathcal{C} \rightarrow \mathcal{D}$ is an equivalence of stable simplicial categories. Then it follows that $f_{!}^{\text {cf }}$ is a DK-equivalence of spectral categories, as is its restriction to the stably representable objects.

Proposition 4.11 Let $\mathcal{A}$ be a spectral category. Then there are natural equivalences of compactly generated stable $\infty$-categories

$$
\mathrm{N}\left(\operatorname{Fun}_{\mathcal{S}}\left(\mathcal{A}^{\mathrm{op}}, \mathcal{S}\right)^{\mathrm{c}}\right)\left[W^{-1}\right] \simeq \operatorname{Ind}\left(\Psi_{\text {tri }} \mathcal{A}\right) \simeq \operatorname{Fun}^{\mathrm{ex}}\left(\Psi_{\text {tri }} \mathcal{A}^{\mathrm{op}}, \mathcal{S}_{\infty}\right) .
$$


Proof The first equivalence follows from the definition of $\Psi_{\text {tri }} \mathcal{A}$ as the smallest stable subcategory of the stable $\infty$-category $\mathrm{N}\left(\operatorname{Fun}_{\mathcal{S}}\left(\mathcal{A}^{\mathrm{op}}, \mathcal{S}\right)^{\mathrm{c}}\right)\left[W^{-1}\right]$ containing the representables, together with the observations that $\mathrm{N}\left(\operatorname{Fun}_{\mathcal{S}}\left(\mathcal{A}^{\mathrm{op}}, \mathcal{S}\right)^{\mathrm{c}}\right)\left[W^{-1}\right]$ is compactly generated with compact objects

$$
\Psi_{\text {perf }} \mathcal{A} \simeq \mathrm{N}\left(\operatorname{Fun}_{\mathcal{S}}\left(\mathcal{A}^{\text {op }}, \mathcal{S}\right)^{\mathrm{c}}\right)\left[W^{-1}\right]^{\omega}
$$

and $\operatorname{Ind}\left(\Psi_{\text {tri }} \mathcal{A}\right) \simeq \operatorname{Ind}\left(\Psi_{\text {perf }} \mathcal{A}\right)$ (as Ind-categories are automatically idempotent-complete). The second equivalence follows immediately from Proposition 3.2.

\subsection{The triangulated and Morita localizations}

Let

$$
M: \mathrm{N}\left(\left(\mathrm{Cat}_{\mathcal{S}}\right)^{\mathrm{c}}\right)\left[W^{-1}\right] \longrightarrow \mathrm{N}\left(\left(\mathrm{Cat}_{\mathcal{S}}\right)^{\mathrm{c}}\right)\left[W^{-1}\right]
$$

denote the composite functor

$$
\mathrm{N}\left(\left(\mathrm{Cat}_{\mathcal{S}}\right)^{\mathrm{c}}\right)\left[W^{-1}\right] \stackrel{\Psi_{\text {tri }}}{\longrightarrow} \mathrm{Cat}_{\infty}^{\mathrm{ex}} \stackrel{\mathrm{N}(\Upsilon)}{\longrightarrow} \mathrm{N}\left(\left(L^{H} \mathrm{Cat}_{\mathcal{S}}\right)^{\mathrm{fib}}\right) \simeq \mathrm{N}\left(\left(\mathrm{Cat}_{\mathcal{S}}\right)^{\mathrm{c}}\right)\left[W^{-1}\right]
$$

As the previous proposition suggests, $M \mathcal{A}$ is essentially the same as the pretriangulated spectral closure $\widehat{\mathcal{A}}_{\text {tri }}$ of $\mathcal{A}$.

Proposition 4.13 There is an equivalence

$$
\widehat{\mathcal{A}}_{\text {tri }} \simeq M \mathcal{A}
$$

in $\mathrm{N}\left(\left(\mathrm{Cat}_{\mathcal{S}}\right)^{\mathrm{c}}\right)\left[W^{-1}\right]$, natural in spectral categories $\mathcal{A}$.

Proof By Proposition 4.11, we have natural equivalences

$$
\mathrm{N}\left(\operatorname{Fun}_{\mathcal{S}}\left(\mathcal{A}^{\mathrm{op}}, \mathcal{S}\right)^{\mathrm{c}}\right)\left[W^{-1}\right]^{\omega} \simeq \Psi_{\text {perf }} \mathcal{A} \simeq \operatorname{Fun}^{\mathrm{ex}}\left(\Psi_{\text {tri }} \mathcal{A}^{\mathrm{op}}, \mathcal{S}_{\infty}\right)^{\omega} .
$$

These allows us to identify the smallest stable subcategory $\Psi_{\text {tri }} \mathcal{A} \subseteq \Psi_{\text {perf }} \mathcal{A}$ spanned by the representable functors $\widehat{a}: \mathcal{A}^{\text {op }} \rightarrow \mathcal{S}$ with the stably representable functors $\Sigma^{\infty} \widehat{a}: \Psi_{\text {tri }} \mathcal{A}^{\text {op }} \rightarrow \mathcal{S}_{\infty}$.

There is a natural transformation $\eta$ : id $\rightarrow M$ which can be described as follows: On the level of spectral categories, the Yoneda embedding

$$
\mathcal{A} \longrightarrow \widehat{\mathcal{A}}
$$

factors through the inclusion of the essentially small full spectral subcategory

$$
\widehat{\mathcal{A}}_{\text {tri }} \longrightarrow \widehat{\mathcal{A}} \text {. }
$$


The result is a natural transformation id $\rightarrow \widehat{(-)}_{\text {tri }}$ of endofunctors of Cat $\mathcal{S}$. By Proposition 4.13, there is a natural equivalence

$$
\widehat{\mathcal{A}}_{\text {tri }} \stackrel{\sim}{\longrightarrow} \Upsilon\left(\Psi_{\text {tri }} \mathcal{A}\right)=M \mathcal{A}
$$

in $\mathrm{N}\left(\left(\mathrm{Cat}_{\mathcal{S}}\right)^{\mathrm{c}}\right)\left[W^{-1}\right]$; composing with this natural equivalence gives the desired natural transformation $\eta$ : id $\rightarrow M$.

Proposition 4.14 For any spectral category $\mathcal{A}, \eta_{\mathcal{A}}: \mathcal{A} \rightarrow M \mathcal{A}$ is fully faithful.

Proof By Yoneda's Lemma, mapping spectra in $M \mathcal{A}$ between stably representable objects are given by mapping spectra between the representing spectrum objects, giving an equivalence

$$
\operatorname{Map}_{M \mathcal{A}}\left(\eta_{\mathcal{A}}(a), \eta_{\mathcal{A}}(b)\right)_{n} \simeq \operatorname{map}_{\Psi_{\mathrm{tri}} \mathcal{A}}\left(\widehat{a}, \Sigma^{n} \widehat{b}\right) .
$$

Since $\Psi_{\text {tri }} \mathcal{A}$ is a stable $\infty$-category of spectral functors, Yoneda's Lemma also gives an equivalence

$$
\operatorname{Map}_{\mathcal{A}}(a, b)_{n} \simeq \operatorname{map}_{\Psi_{\mathrm{tri}} \mathcal{A}}\left(\widehat{a}, \Sigma^{n} \widehat{b}\right) .
$$

Hence $\operatorname{Map}_{\mathcal{A}}(a, b) \simeq \operatorname{Map}_{M_{\mathcal{A}}}\left(\eta_{\mathcal{A}}(a), \eta_{\mathcal{A}}(b)\right)$.

Proposition 4.15 The functor $\eta_{\mathcal{A}}: \mathcal{A} \rightarrow M \mathcal{A}$ is essentially surjective if and only if $\mathcal{A}$ is stable.

Proof Indeed, $\mathcal{A} \rightarrow M \mathcal{A}$ is essentially surjective if and only if $\Omega^{\infty} \mathcal{A} \rightarrow \Omega^{\infty} M \mathcal{A}$ is essentially surjective, which is the case if and only if $\mathcal{A}$ is already stable.

Combining Propositions 4.14 and 4.15, we obtain the following corollary.

Corollary 4.16 The functor $\eta_{\mathcal{A}}: \mathcal{A} \rightarrow M \mathcal{A}$ is an equivalence of spectral categories if and only if $\mathcal{A}$ is a stable spectral category.

Next, we want to verify that $M$ is a localization.

Proposition 4.17 The pair of natural transformations $\eta_{M \mathcal{A}}, M \eta_{\mathcal{A}}: M \mathcal{A} \rightarrow M^{2} \mathcal{A}$ induce a homotopy commutative square

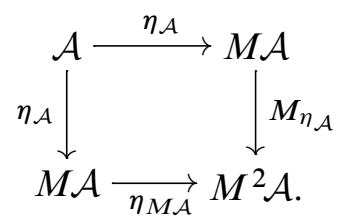


Proof First note that $M \eta_{\mathcal{A}}: M \mathcal{A} \rightarrow M^{2} \mathcal{A}$ sends $x: \widehat{\mathcal{A}} \rightarrow \mathcal{S}_{\infty}$ to the functor

$$
\widehat{\eta_{\mathcal{A}}} x: \widehat{M \mathcal{A}} \longrightarrow \mathcal{S}_{\infty}
$$

induced by homotopy left Kan extension along $\widehat{\eta_{\mathcal{A}}}: \widehat{\mathcal{A}} \rightarrow \widehat{M \mathcal{A}}$. If $x=\operatorname{Map}_{A}(-, a)$ is represented by the object $a$ of $\mathcal{A}$, then the universal properties of representable functors and homotopy left Kan extensions force an equivalence $\widehat{\eta_{\mathcal{A}}}$ ! $x \cong \operatorname{Map}\left(-, \eta_{\mathcal{A}}(a)\right)$, so that $\widehat{\eta_{\mathcal{A}}} x$ is represented by $\eta_{\mathcal{A}}(a)$. It follows that the restrictions of $\eta_{M_{\mathcal{A}}}$ and $M \eta_{\mathcal{A}}$ to $\mathcal{A}$ are equivalent.

Corollary 4.18 The spectral functors $\eta_{M_{\mathcal{A}}}$ and $M \eta_{\mathcal{A}}$ are equivalent. In particular, both $\eta_{M \mathcal{A}}$ and $M \eta_{\mathcal{A}}$ are equivalences.

Proof Since $\mathcal{A}$ generates $M \mathcal{A}$ under finite homotopy colimits and desuspensions, it suffices to show that $M \eta_{\mathcal{A}}$ preserves finite homotopy colimits and desuspensions. The fact that $M \eta_{\mathcal{A}}$ preserves finite homotopy colimits follows from the fact that $M \eta_{\mathcal{A}}$ is a homotopy left Kan extension along $\widehat{\eta_{\mathcal{A}}}$. But suspension is an example of a finite homotopy colimit, so we have that $M \eta_{\mathcal{A}}(\Sigma x) \simeq \Sigma M \eta_{\mathcal{A}}(x)$. Hence $M \eta_{\mathcal{A}}(x) \simeq$ $\Sigma M \eta_{\mathcal{A}}\left(\Sigma^{-1} x\right)$, and as $M^{2} \mathcal{A}$ is stable we see that $M \eta_{\mathcal{A}}\left(\Sigma^{-1} x\right) \simeq \Sigma^{-1} M \eta_{\mathcal{A}}(x)$. The final statement is a consequence of Corollary 4.16 and the fact that $M \mathcal{A}$ is a stable spectral category.

Corollary 4.19 The functor $M$ defines a localization of $\mathrm{N}\left(\left(\mathrm{Cat}_{\mathcal{S}}\right)^{\mathrm{c}}\right)\left[W^{-1}\right]$ with essential image the stable spectral categories.

Proof This follows from the previous proposition and corollary by [47, 5.2.7.4].

To see that we have an accessible localization, we need the following proposition:

Proposition 4.20 Let $\mathcal{C} \simeq \operatorname{colim}_{i} \mathcal{C}_{i}$ be a filtered colimit of stable $\infty$-categories. Then there is an equivalence of spectral categories

$$
\Upsilon(\mathcal{C}) \simeq \operatorname{colim}_{i} \Upsilon\left(\mathcal{C}_{i}\right)
$$

Proof We must show that the natural map

$$
\operatorname{colim}_{i} \Upsilon\left(\mathcal{C}_{i}\right) \longrightarrow \Upsilon(\mathcal{C})
$$

is a DK-equivalence of spectral categories. Since $\mathcal{C}$ and the $\mathcal{C}_{i}$ are all stable spectral categories and $\Omega^{\infty}$ and $N$ commute with filtered colimits, this follows from Propositions 4.5 and 4.8 . 
Following Definition 2.7, we make the following definitions.

Definition 4.21 A map of small spectral $\infty$-categories $f: \mathcal{A} \rightarrow \mathcal{B}$ is:

- A triangulated equivalence if $\Psi_{\text {tri }} f: \Psi_{\text {tri }} \mathcal{A} \rightarrow \Psi_{\text {tri }} \mathcal{B}$ is an equivalence of (stable) $\infty$-categories, and

- A Morita equivalence if $\Psi_{\text {perf }} f: \Psi_{\text {perf }} \mathcal{A} \rightarrow \Psi_{\text {perf }} \mathcal{B}$ is an equivalence of (idempotent-complete) stable $\infty$-categories.

Assembling the work of this section we obtain the following two results:

Theorem 4.22 The functor

$$
\Psi_{\text {tri }}(-): \mathrm{N}\left(\left(\mathrm{Cat}_{\mathcal{S}}\right)^{\mathrm{c}}\right)\left[W^{-1}\right] \longrightarrow \mathrm{Cat}_{\infty}^{\mathrm{ex}}
$$

admits a fully faithful and accessible right adjoint

$$
\Upsilon: \mathrm{Cat}_{\infty}^{\mathrm{ex}} \longrightarrow \mathrm{N}\left(\left(\mathrm{Cat}_{\mathcal{S}}\right)^{\mathrm{c}}\right)\left[W^{-1}\right]
$$

That is, the $\infty$-category of stable $\infty$-categories is an accessible localization of the $\infty$-category of spectral categories obtained by inverting the triangulated equivalences.

Proof The result follows from Proposition 4.20 and the factorization of $M$ given in (4.12).

Recall that we have a stable idempotent completion functor Idem: $\mathrm{Cat}_{\infty}^{\mathrm{ex}} \rightarrow \mathrm{Cat}_{\infty}^{\mathrm{perf}}$. Since Idem is left adjoint to the (fully faithful) inclusion $\mathrm{Cat}_{\infty}^{\text {perf }} \rightarrow \mathrm{Cat}_{\infty}^{\mathrm{ex}}$, Cat $t_{\infty}^{\text {perf }}$ is the localization of $\mathrm{Cat}_{\infty}^{\mathrm{ex}}$ obtain by inverting idempotent completion maps. Further, recall that there is an equivalence Idem $\circ \Psi_{\text {tri }} \simeq \Psi_{\text {perf }}$.

Theorem 4.23 The functor

$$
\Psi_{\text {perf }}: \mathrm{N}\left(\left(\mathrm{Cat}_{\mathcal{S}}\right)^{\mathrm{c}}\right)\left[W^{-1}\right] \longrightarrow \mathrm{Cat}_{\infty}^{\text {perf }}
$$

admits a fully faithful and accessible right adjoint

$$
\Upsilon: \mathrm{Cat}_{\infty}^{\text {perf }} \longrightarrow \mathrm{N}\left(\left(\mathrm{Cat}_{\mathcal{S}}\right)^{\mathrm{c}}\right)\left[W^{-1}\right] .
$$

That is, the $\infty$-category of idempotent-complete stable $\infty$-categories is an accessible localization of the $\infty$-category of spectral categories obtained by inverting the Morita equivalences.

Proof The $\infty$-category of idempotent-complete stable $\infty$-categories is a localizing subcategory of the $\infty$-category of stable $\infty$-categories, and idempotent-completion is an accessible functor as the inclusion $\Psi_{\text {tri }} \rightarrow \Psi_{\text {perf }}$ preserves filtered colimits. 
Remark 4.24 Theorems 4.22 and 4.23 imply that computing the localizations of the model category structure on spectral categories from Corollary 2.4 at the triangulated and Morita equivalences (as discussed in Remark 4.3) and passing to the simplicial nerve also yields the $\infty$-categories $\mathrm{Cat}_{\infty}^{\mathrm{ex}}$ and $\mathrm{Cat}_{\infty}^{\text {perf }}$ respectively.

We conclude the section with the promised applications of the theory. First, the fact that we have accessible localizations provides the following corollary about the structure of $\mathrm{Cat}_{\infty}^{\mathrm{ex}}$ and $\mathrm{Cat}_{\infty}^{\text {perf }}$.

Corollary 4.25 The $\infty$-categories $\mathrm{Cat}_{\infty}^{\mathrm{ex}}$ and $\mathrm{Cat}_{\infty}^{\mathrm{perf}}$ are compactly generated, complete and cocomplete.

We will use the comparison above to lift small stable $\infty$-categories to spectral categories. To this end, we make the following definition.

Definition 4.26 Let $\mathcal{A}$ and $\mathcal{B}$ be small idempotent-complete stable $\infty$-categories. We write $\operatorname{rep}(\mathcal{B}, \mathcal{A})=\Upsilon\left(\operatorname{Fun}^{\mathrm{ex}}(\mathcal{B}, \mathcal{A})\right)$ for the small pretriangulated spectral category associated to the small stable $\infty$-category of exact functors from $\mathcal{B}$ to $\mathcal{A}$.

Corollary 4.27 Let $\mathcal{A}$ and $\mathcal{B}$ be idempotent-complete small stable $\infty$-categories and let $\Upsilon(A)$ and $\Upsilon(B)$ be spectral categories lifting $\mathcal{A}$ and $\mathcal{B}$. Then

$$
\mathrm{N}(\operatorname{rep}(\mathcal{A}, \mathcal{B})) \simeq \operatorname{Fun}^{\mathrm{ex}}(\mathcal{A}, \mathcal{B})
$$

is equivalent to the $\infty$-category of right-compact $\Upsilon(A)^{\mathrm{op}} \wedge \Upsilon(B)$-modules.

Proof This follows from Theorem 4.23 and Corollary 3.3.

We also record the following result concerning lifting diagrams of small stable $\infty-$ categories to diagrams of spectral categories.

Proposition 4.28 Let $I$ be a small category. Given a diagram $\mathcal{D}$ of small stable $\infty$-categories indexed by $\mathrm{N}(I)$, there exists an $I$-diagram of pretriangulated spectral categories $\widetilde{\mathcal{D}}$ lifting $\mathcal{D}$.

Proof This is a consequence of [47, 4.2.4.4]. Given a diagram of small stable $\infty-$ categories, the equivalence in Theorem 4.22 gives rise to a diagram in the localization of $\mathrm{N}\left(\left(\mathrm{Cat}_{\mathcal{S}}\right)^{\mathrm{c}}\right)\left[W^{-1}\right]$. Including the localization into $\mathrm{N}\left(\left(\mathrm{Cat}_{\mathcal{S}}\right)^{\mathrm{c}}\right)\left[W^{-1}\right]$, we now obtain a diagram in $\mathrm{N}\left(\left(\mathrm{Cat}_{\mathcal{S}}\right)^{\mathrm{c}}\right)\left[W^{-1}\right] \simeq \mathrm{N}\left(\left(\mathrm{Cat}_{\mathcal{S}}\right)^{\mathrm{cf}}\right)$ and we can use [47, 4.2.4.4] to lift this to a rigid diagram in $\mathrm{Cat}_{\mathcal{S}}$. 


\section{Exact sequences}

In this section we discuss the various definitions of exact sequence, relating notions for triangulated categories, spectral categories and stable $\infty$-categories. Arguably the most fundamental definition is that of an exact sequence of triangulated categories, as it turns out that exact sequences in both spectral and stable $\infty$-categories can be detected on the level of the homotopy category. Recall that a sequence of triangulated categories

$$
\mathcal{A} \longrightarrow \mathcal{B} \longrightarrow \mathcal{C}
$$

is called exact if the composite is zero, the functor $\mathcal{A} \rightarrow \mathcal{B}$ is fully faithful, and the induced functor from the Verdier quotient $\mathcal{B} / \mathcal{A}$ to $\mathcal{C}$ is cofinal, ie it becomes an equivalence after idempotent completion. Said differently, a triangulated functor $\mathcal{C}^{\prime} \rightarrow \mathcal{C}$ is cofinal if every object of $\mathcal{C}$ is a summand of an object of $\mathcal{C}^{\prime}$. The purpose of this section is to develop analogues of these notions for stable $\infty$-categories.

\subsection{The Verdier quotient as the cofiber in $\mathrm{Cat}_{\infty}^{\text {perf }}$}

Let $\kappa$ denote an infinite regular cardinal. We recall the following terminology from [47, Section 5.3.4].

Definition 5.1 Let $\mathcal{A}$ be an $\infty$-category. We say that $\mathcal{A}$ is $\kappa$-cocomplete if $\mathcal{A}$ admits all $\kappa$-small colimits.

Most of the small $\infty$-categories which arise in this paper can be realized as the full subcategory $\mathcal{C}^{\kappa} \subset \mathcal{C}$ of $\kappa$-compact objects in a stable presentable $\infty$-category $\mathcal{C}$. In this case, we can reconstruct $\mathcal{C}$ itself as $\operatorname{Ind}_{\kappa}\left(\mathcal{C}^{\kappa}\right)$, which formally adjoins $\kappa-$ filtered colimits. To make this precise, we recall the notions of $\kappa$-filtered $\infty$-category, $\kappa$-filtered colimit and $\kappa$-continuous functor.

Definition 5.2 An $\infty$-category $\mathcal{C}$ is $\kappa$-filtered if every map $K \rightarrow \mathcal{C}$ from a $\kappa$-small simplicial set $K$ extends to a functor $K^{\triangleright} \rightarrow \mathcal{C}$ (see [47, 2.1.4.2] for the cone notation). A simplicial set $K$ is $\kappa$-filtered if there exists a categorical equivalence $K \rightarrow \mathcal{C}$ for some $\kappa$-filtered $\infty$-category $\mathcal{C}$. Lastly, a $\kappa$-filtered colimit is a colimit indexed by a $\kappa$-filtered simplicial set.

Definition 5.3 Let $\mathcal{A}$ and $\mathcal{B}$ be $\infty$-categories and let $f: \mathcal{A} \rightarrow \mathcal{B}$ be a functor. We say that $f$ is $\kappa$-continuous if $f$ preserves $\kappa$-filtered colimits. 
We write $\mathrm{Cat}_{\infty}^{\mathrm{ex}(\kappa)}$ for the $\infty$-category of small $\kappa$-cocomplete stable $\infty$-categories and $\kappa$-small colimit-preserving functors thereof; note that if $\kappa>\omega$, any small $\kappa-$ cocomplete stable $\infty$-category $\mathcal{A}$ is necessarily idempotent complete [47, 5.4.2.4]. Given a small $\kappa$-cocomplete stable $\infty$-category $\mathcal{A}$, the $\infty$-category $\operatorname{Ind}_{\kappa}(\mathcal{A})$ is a $\kappa-$ compactly generated stable $\infty$-category such that $\operatorname{Idem}(\mathcal{A}) \simeq \operatorname{Ind}_{\kappa}(\mathcal{A})^{\kappa}[47$, 5.5.7.8, 5.5.7.10].

In fact, provided $\kappa>\omega$, restriction to subcategories of $\kappa$-compact objects determines an equivalence between the $\infty$-category of $\kappa$-compactly generated stable $\infty$-categories $\mathcal{P r}_{\text {St } \kappa}^{\mathrm{L}}$ and the $\infty$-category $\mathrm{Cat}_{\infty}^{\mathrm{ex}(\kappa)}$ of small $\kappa$-cocomplete stable $\infty$-categories, with inverse $\operatorname{Ind}_{\kappa}[47,5.5 .7 .10]$. As a consequence, Corollary 4.25 implies that the $\infty$-category of $\kappa$-compactly generated stable $\infty$-categories is cocomplete.

We now define an analogue of the Verdier quotient of triangulated categories on the level of stable $\infty$-categories. Specifically, if $\mathcal{A} \rightarrow \mathcal{B}$ is a fully faithful functor of stable $\infty$-categories, then $\operatorname{Ho}(\mathcal{A}) \rightarrow \operatorname{Ho}(\mathcal{B})$ is a fully faithful functor of triangulated categories, and we may form the usual Verdier quotient $\operatorname{Ho}(\mathcal{B}) / \operatorname{Ho}(\mathcal{A})$. This is defined as the initial triangulated category $\operatorname{Ho}(\mathcal{B}) / \operatorname{Ho}(\mathcal{A})$ equipped with a triangulated functor $\operatorname{Ho}(\mathcal{B}) \rightarrow \operatorname{Ho}(\mathcal{B}) / \operatorname{Ho}(\mathcal{A})$ such that the composite $\operatorname{Ho}(\mathcal{A}) \rightarrow \operatorname{Ho}(\mathcal{B}) \rightarrow \operatorname{Ho}(\mathcal{B} / \mathcal{A})$ is trivial $[57,2.1 .8]$.

Definition 5.4 Let $f: \mathcal{A} \rightarrow \mathcal{B}$ be a fully faithful functor of presentable stable $\infty-$ categories (this means that $f$ preserves colimits). The Verdier quotient $\mathcal{B} / \mathcal{A}$ of $\mathcal{B}$ by $\mathcal{A}$ is the cofiber of $f$ in the $\infty$-category $\mathcal{P r}_{\text {St }}^{\mathrm{L}}$ of presentable stable $\infty$-categories.

It is useful to identify the Verdier quotient in terms of a Bousfield localization; specifically, we will see that the Verdier quotient is the Bousfield localization of $\mathcal{B}$ at the arrows with cofiber in $\mathcal{A}$.

Lemma 5.5 Let $\mathcal{C}$ be a presentable $\infty$-category and $S$ be a strongly saturated class of arrows of $\mathcal{C}$. Then $S$ is of small generation if and only if the full subfunctor

$$
\operatorname{Fun}_{S}^{\mathrm{L}}(\mathcal{C},-) \subseteq \operatorname{Fun}^{\mathrm{L}}(\mathcal{C},-): \mathcal{P r}^{\mathrm{L}} \longrightarrow \widehat{\operatorname{Cat}}_{\infty}
$$

of Fun $^{\mathrm{L}}(\mathcal{C},-)$, spanned by those colimit-preserving functors $\mathcal{C} \rightarrow \mathcal{D}$ which carry the arrows in $S$ to equivalences in $\mathcal{D}$, is corepresentable by a presentable $\infty$-category $\mathcal{C}^{\prime}$. Moreover, in this case, $\mathcal{C}^{\prime} \simeq S^{-1} \mathcal{C}$.

Proof If $S$ is of small generation then $S^{-1} \mathcal{C}$ is presentable and corepresents the functor $\operatorname{Fun}_{S}^{\mathrm{L}}(\mathcal{C},-)$ by $[47,5.5 .4 .14,5.5 .4 .20]$. Conversely, if this functor is corepresentable by $\mathcal{C}^{\prime}$ then the identity $\mathcal{C}^{\prime} \rightarrow \mathcal{C}^{\prime}$ determines a colimit-preserving functor $\mathcal{C} \rightarrow \mathcal{C}^{\prime}$. 
Let $T$ be the class of arrows in $\mathcal{C}$ which become invertible in $\mathcal{C}^{\prime}$, and note that $S \subseteq T$, $T$ is strongly saturated [47, 5.5.4.10] and $T$ is of small generation [47, 5.5.4.16] (the last claim uses the fact that the equivalences in $\mathcal{C}^{\prime}$ is the strongly saturated class generated by the identity of the initial object of $\mathcal{C}^{\prime}$, which follows from [47, 5.5.4.5, 5.5.4.6]). Thus $T^{-1} \mathcal{C} \simeq \mathcal{C}^{\prime}$, so $\mathcal{C}^{\prime}$ also corepresents the functor $\operatorname{Fun}_{T}^{\mathrm{L}}(\mathcal{C},-)$, showing that a colimit-preserving functor $\mathcal{C} \rightarrow \mathcal{D}$ inverts the arrows of $S$ if and only if it inverts the arrows of $T$. Since $S$ is strongly saturated, we conclude that $S=T$.

The preceding lemma now allows us to characterize the cofiber as a localization.

Proposition 5.6 Let $\mathcal{A} \rightarrow \mathcal{B}$ be a fully faithful functor of presentable stable $\infty-$ categories and let $S$ denote the collection of arrows in $\mathcal{B}$ whose cones lie in the essential image of $\mathcal{A}$. Then $S$ is a strongly saturated class of maps in $\mathcal{B}$ of small generation, and the Verdier quotient $\mathcal{B} / \mathcal{A}$ is equivalent to the Bousfield localization $S^{-1} \mathcal{B}$.

Proof Let $\mathcal{C}$ be a presentable stable $\infty$-category, and note that a colimit-preserving functor $\mathcal{B} \rightarrow \mathcal{C}$ sends the arrows in $S$ to equivalences in $\mathcal{C}$ if and only if its restriction to $\mathcal{A}$ is trivial. We therefore may identify

$$
\operatorname{Fun}^{\mathrm{L}}(\mathcal{B} / \mathcal{A}, \mathcal{C}) \subseteq \operatorname{Fun}^{\mathrm{L}}(\mathcal{B}, \mathcal{C})
$$

with the full subcategory spanned by those colimit-preserving functors $\mathcal{B} \rightarrow \mathcal{C}$ which send the arrows in $S$ to equivalences in $\mathcal{C}$. It follows from Lemma 5.5 that $\mathcal{B} / \mathcal{A} \simeq$ $T^{-1} \mathcal{B}$, where $T$ is the strongly saturated class of arrows of $\mathcal{B}$ which become equivalences in $\mathcal{B} / \mathcal{A}$.

We now show that $S$ is strongly saturated, so that $S=T$. First, suppose given a cofiber sequence $X \rightarrow Y \rightarrow Z$ in $\mathcal{B}$ such that $Z$ lies in the essential image of $\mathcal{A}$, and let $X \rightarrow X^{\prime}$ be any map. Then the cofiber of $X^{\prime} \rightarrow X^{\prime} \sqcup_{X} Y$ is equivalence to $Z$, so is also in the essential image of $\mathcal{A}$. Second, suppose given a diagram $f_{\alpha}: X_{\alpha} \rightarrow Y_{\alpha}$ in Fun $\left(\Delta^{1}, \mathcal{B}\right)$ with colimit $f: X \rightarrow Y$, and suppose that the cofibers $Z_{\alpha}$ of each $f_{\alpha}$ lies in the essential image of $\mathcal{A}$. Commuting colimits implies that the cofiber $Z$ of $f$ is computed as the colimit of the $Z_{\alpha}$, and this lies in the essential image of $\mathcal{A}$ since $\mathcal{A}$ is closed under colimits and the functor $\mathcal{A} \rightarrow \mathcal{B}$ preserves colimits. Lastly, suppose $h=g \circ f$ is a composite of $f: X \rightarrow Y$ followed by $g: Y \rightarrow Z$, and write $Y / X$, $Z / Y$ and $Z / X$ for the cofibers of $f, g$ and $h$, respectively. Then we have a cofiber sequence $Y / X \rightarrow Z / X \rightarrow Z / Y$, so if any two lie in the essential image of $\mathcal{A}$ then so does the third.

In fact, we can be more precise about a generating set for the local equivalences: 
Proposition 5.7 Let $i: \mathcal{A} \rightarrow \mathcal{B}$ be a fully faithful inclusion of $\kappa$-compactly generated stable $\infty$-categories which preserves $\kappa$-compact objects, let $S$ be the (small) collection of arrows of $\mathcal{B}^{\kappa}$ whose cofibers lie in the image of $\mathcal{A}^{\kappa}$ and let $T$ be the (large) collection of arrows of $\mathcal{B}$ whose cofibers lie in the image of $\mathcal{A}$. Then the natural map

$$
S^{-1} \mathcal{B} \longrightarrow T^{-1} \mathcal{B} \simeq \mathcal{B}\left[T^{-1}\right] \simeq \mathcal{B} / \mathcal{A}
$$

is an equivalence of $\infty$-categories, where $S^{-1} \mathcal{B}$ and $T^{-1} \mathcal{B}$ denote the subcategories of local objects.

Proof Without loss of generality we may identify $\mathcal{A}$ with its essential image in $\mathcal{B}$, so that an arrow $f: X \rightarrow Y$ is in $T$ if and only if any cofiber $Z$ of $f$ lies in $\mathcal{A}$. By [47, 5.5.4.15] it suffices to show that $T \subseteq \bar{S}$, the strongly saturated class of arrows of $\mathcal{B}$ generated by $S$ (see $[47,5.5 .4 .5]$ ). To see this, let

$$
X \stackrel{f}{\longrightarrow} Y \stackrel{g}{\longrightarrow} Z
$$

be a cofiber sequence in $\mathcal{B}$ such that $Z$ is in $\mathcal{A}$. Then $Z=\operatorname{colim}_{\alpha} Z_{\alpha}$ is a $\kappa$-filtered colimit of objects $Z_{\alpha} \in \mathcal{A}^{\kappa} \subset \mathcal{B}^{\kappa}$ and $Y=\operatorname{colim}_{\alpha} Y_{\alpha}$ is a $\kappa$-filtered colimit of objects $Y_{\alpha}=Y \times{ }_{Z} Z_{\alpha}$. Now $Y_{\alpha}$ may not be $\kappa$-compact, so write $Y_{\alpha}=\operatorname{colim}_{\beta} Y_{\alpha \beta}$ for some $Y_{\alpha \beta} \in \mathcal{B}^{\kappa}$ and consider the resulting diagram of cofiber sequences

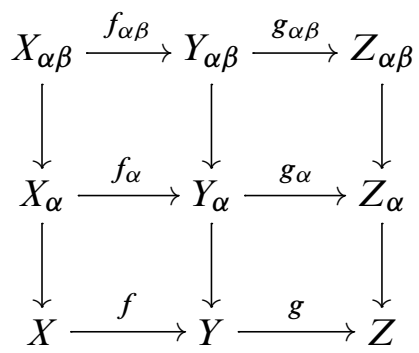

in which the lower right and upper left squares are cartesian, which implies that these two squares are also cocartesian and that the maps $X_{\alpha} \rightarrow X$ and $Z_{\alpha \beta} \rightarrow Z_{\alpha}$ are equivalences. Hence $Z_{\alpha \beta} \in \mathcal{A}^{\kappa} \subseteq \mathcal{B}^{\kappa}$ and we conclude that $g_{\alpha \beta}$ and therefore $f_{\alpha \beta}$ as well are maps in $\mathcal{B}^{\kappa}$; in particular, $f_{\alpha \beta}$ is an arrow in $S$. It follows from [47, 5.5.4.5] that the pushout $f_{\alpha}$ of $f_{\alpha \beta}$ along $X_{\alpha \beta} \rightarrow X_{\alpha} \simeq X$ is in $\bar{S}$, and we see from [47, 5.5.4.12] that $f \simeq \operatorname{colim}_{\alpha} f_{\alpha}: X \simeq \operatorname{colim}_{\alpha} X_{\alpha} \rightarrow \operatorname{colim} Y_{\alpha} \simeq Y$ is then also in $\bar{S}$.

Definition 5.4 leads to the following definition of an exact sequence.

Definition 5.8 A sequence of presentable stable $\infty$-categories $\mathcal{A} \rightarrow \mathcal{B} \rightarrow \mathcal{C}$ is exact if the composite is trivial, $\mathcal{A} \rightarrow \mathcal{B}$ is fully faithful and the map $\mathcal{B} / \mathcal{A} \rightarrow \mathcal{C}$ is an equivalence. 
Somewhat surprisingly, as a consequence of the hypothesis of stability we can detect exact sequences on the level of homotopy categories, despite the fact that functors which are fully faithful on homotopy categories are not typically fully faithful as functors of $\infty$-categories. The following proposition connects the $\infty$-categorical Verdier quotient of Definition 5.4 to the Verdier quotient of the triangulated homotopy categories.

Proposition 5.9 Let $\mathcal{A} \rightarrow \mathcal{B}$ be a fully faithful inclusion of presentable stable $\infty-$ categories. Then the natural map $\operatorname{Ho}(\mathcal{B}) / \operatorname{Ho}(\mathcal{A}) \rightarrow \operatorname{Ho}(\mathcal{B} / \mathcal{A})$ is an equivalence.

Proof By construction, $\mathcal{B} / \mathcal{A} \subseteq \mathcal{B}$ is the full subcategory on those objects $b$ such that $\operatorname{map}(a, b) \simeq *$ for all objects $a$ in the image of $\mathcal{A}$. This shows that, as full subcategories of $\operatorname{Ho}(\mathcal{B}), \operatorname{Ho}(\mathcal{B} / \mathcal{A}) \subseteq \operatorname{Ho}(\mathcal{B}) / \operatorname{Ho}(\mathcal{A})$. Conversely, if $b$ is in $\operatorname{Ho}(\mathcal{B}) / \operatorname{Ho}(\mathcal{A})$, then $\pi_{0} \operatorname{map}(a, b) \simeq *$ for each object $a$ in the image of $\mathcal{A}$, and we claim that in fact $\operatorname{map}(a, b) \simeq *$. Indeed, $\mathcal{A}$ is a stable subcategory of $\mathcal{B}$, so that $\pi_{n} \operatorname{map}(a, b) \simeq$ $\pi_{0} \operatorname{map}\left(\Sigma^{n} a, b\right) \simeq *$. Hence $\operatorname{Ho}(\mathcal{B}) / \operatorname{Ho}(\mathcal{A}) \subseteq \operatorname{Ho}(\mathcal{B} / \mathcal{A})$ as well.

The argument for the previous proposition also implies the following characterization of fully faithful maps; note that here we do not need the hypothesis that the stable $\infty$-categories are presentable, as we are not working with localizations.

Proposition 5.10 A map of stable $\infty$-categories $\mathcal{A} \rightarrow \mathcal{B}$ is fully faithful if and only if $\operatorname{Ho}(\mathcal{A}) \rightarrow \operatorname{Ho}(\mathcal{B})$ is fully faithful.

Corollary 5.11 A map of stable $\infty$-categories $\mathcal{A} \rightarrow \mathcal{B}$ is an equivalence if and only if $\operatorname{Ho}(\mathcal{A}) \rightarrow \operatorname{Ho}(\mathcal{B})$ is an equivalence.

As we are predominantly interested in sequences of small $\infty$-categories, we will now extend Definition 5.8 to the $\infty$-category $\mathrm{Cat}_{\infty}^{\mathrm{ex}(\kappa)}$.

Definition 5.12 A sequence of $\kappa$-cocomplete small stable $\infty$-categories and $\kappa$-small colimit preserving functors $\mathcal{A} \rightarrow \mathcal{B} \rightarrow \mathcal{C}$ is exact if the sequence

$$
\operatorname{Ind}_{\kappa}(\mathcal{A}) \longrightarrow \operatorname{Ind}_{\kappa}(\mathcal{B}) \longrightarrow \operatorname{Ind}_{\kappa}(\mathcal{C})
$$

is an exact sequence of presentable stable $\infty$-categories.

Although we've defined exact sequences in $\mathrm{Cat}_{\infty}^{\mathrm{ex}(\kappa)}$ to be those sequences which are exact in $\mathcal{P r}_{\text {St }}^{\mathrm{L}}$, we can give an intrinsic description. Just as in the presentable case, the quotient $\mathcal{B} / \mathcal{A}$ will denote the cofiber of the fully faithful inclusion $\mathcal{A} \rightarrow \mathcal{B}$ of $\kappa$-cocomplete small stable $\infty$-categories. 
Proposition 5.13 A sequence of $\kappa$-cocomplete small stable $\infty$-categories and $\kappa-$ small colimit preserving functors $\mathcal{A} \rightarrow \mathcal{B} \rightarrow \mathcal{C}$ is exact if and only if the composite is trivial, $\mathcal{A} \rightarrow \mathcal{B}$ is fully faithful, and the resulting map $\mathcal{B} / \mathcal{A} \rightarrow \mathcal{C}$ is an equivalence (after idempotent completion if $\kappa=\omega$ ).

Proof The fully faithful inclusions $\mathcal{A} \subset \operatorname{Ind}_{\kappa}(\mathcal{A})$ and $\mathcal{B} \subset \operatorname{Ind}_{\kappa}(\mathcal{B})$ show that $\mathcal{A} \rightarrow \mathcal{B}$ is fully faithful if and only if $\operatorname{Ind}_{\kappa}(\mathcal{A}) \rightarrow \operatorname{Ind}_{\kappa}(\mathcal{B})$ is fully faithful (for the reverse direction, this follows from the definition of the mapping spaces in $\left.\operatorname{Ind}_{\kappa}(-)\right)$. Thus it remains to check that $\mathcal{B} / \mathcal{A} \rightarrow \mathcal{C}$ is an equivalence upon idempotent completion if and only if $\operatorname{Ind}_{\kappa}(\mathcal{B}) / \operatorname{Ind}_{\kappa}(\mathcal{A}) \simeq \operatorname{Ind}_{\kappa}(\mathcal{B} / \mathcal{A})$. Since $\operatorname{Ind}_{\kappa}$ preserves cofibers, it is enough to check that the equivalence $\operatorname{Ind}_{\kappa}(\mathcal{B}) / \operatorname{Ind}_{\kappa}(\mathcal{A}) \simeq \operatorname{Ind}_{\kappa}(\mathcal{B} / \mathcal{A})$ implies the equivalence $\mathcal{B} / \mathcal{A} \simeq \mathcal{C}$ whenever the latter are idempotent complete. Thus, given a $\kappa$-cocomplete small stable $\infty$-category $\mathcal{D}$ (which we assume is idempotent complete if $\kappa=\omega$ ), we must show that

$$
\operatorname{Fun}^{\operatorname{ex}(\kappa)}(\mathcal{C}, \mathcal{D}) \longrightarrow \operatorname{Fun}^{\mathrm{ex}(\kappa)}(\mathcal{B}, \mathcal{D}) \longrightarrow \operatorname{Fun}^{\mathrm{ex}(\kappa)}(\mathcal{A}, \mathcal{D})
$$

is a fiber sequence of $\infty$-categories. Since $\mathcal{D} \simeq\left(\operatorname{Ind}_{\kappa}(\mathcal{D})\right)^{\kappa}$, by adjunction this is equivalent to the sequence

$$
\operatorname{Fun}^{\mathrm{L}}\left(\operatorname{Ind}_{\kappa}(\mathcal{C}), \operatorname{Ind}_{\kappa}(\mathcal{D})\right) \longrightarrow \operatorname{Fun}^{\mathrm{L}}\left(\operatorname{Ind}_{\kappa}(\mathcal{B}), \operatorname{Ind}_{\kappa}(\mathcal{D})\right) \longrightarrow \operatorname{Fun}^{\mathrm{L}}\left(\operatorname{Ind}_{\kappa}(\mathcal{A}), \operatorname{Ind}_{\kappa}(\mathcal{D})\right)
$$

being a fiber sequence, which it is by assumption.

\subsection{The Thomason-Neeman Localization Theorem}

In fact, we can further reduce to a criterion on the level of homotopy categories. For this, we need the following proposition. In its proof, we take advantage of the detailed study of localization in the context of (well-generated) triangulated categories by Neeman [57; 56] and Krause [46] and the fact that the homotopy category of a presentable stable $\infty$-category is a well-generated triangulated category (see [48, 1.4.4.3] and [45]).

Proposition 5.14 Let $\mathcal{A} \rightarrow \mathcal{B}$ be a fully faithful and $\kappa$-small colimit preserving functor of $\kappa$-cocomplete small stable $\infty$-categories. Then the natural map

$$
\operatorname{Ho}(\mathcal{B}) / \operatorname{Ho}(\mathcal{A}) \longrightarrow \operatorname{Ho}(\mathcal{B} / \mathcal{A})
$$

is an equivalence. In other words, the functor $\mathrm{Ho}(-)$ preserves quotients of fully faithful functors.

Proof We have equivalences

$$
\operatorname{Ho}\left(\operatorname{Ind}_{\kappa}(\mathcal{B})\right) / \operatorname{Ho}\left(\operatorname{Ind}_{\kappa}(\mathcal{A})\right) \simeq \operatorname{Ho}\left(\operatorname{Ind}_{\kappa}(\mathcal{B}) / \operatorname{Ind}_{\kappa}(\mathcal{A})\right) \simeq \operatorname{Ho}\left(\operatorname{Ind}_{\kappa}(\mathcal{B} / \mathcal{A})\right)
$$


where the first equivalence follows from Proposition 5.9 and the last equivalence follows from the fact that $\operatorname{Ind}_{\kappa}$ preserves cofibers. We therefore obtain a commutative (up to natural isomorphism) square

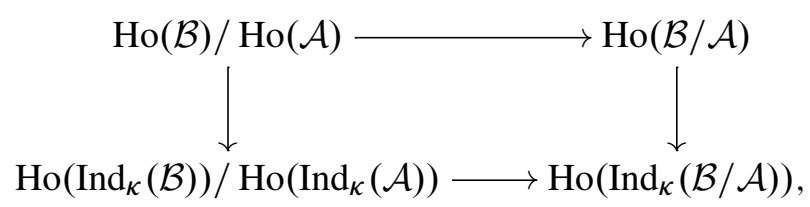

where the right vertical map is fully faithful and the bottom map is an equivalence.

To see that the top vertical map is fully faithful, we use Neeman's generalization of Thomason's Localization Theorem (see [57, 4.4.9] or [56]) to show that the left vertical map is fully faithful. First, $\operatorname{since} \operatorname{Ind}_{\kappa}(\mathcal{A})$ and $\operatorname{Ind}_{\kappa}(\mathcal{B})$ are presentable, the criterion of [45] (characterizing well-generated triangulated categories) and [48, 1.4.4.2] imply that $\operatorname{Ho}\left(\operatorname{Ind}_{\kappa}(\mathcal{B})\right)$ is well-generated and $\left(\operatorname{since}\right.$ the $\operatorname{map} \operatorname{Ind}_{\kappa}(\mathcal{A}) \rightarrow \operatorname{Ind}_{\kappa}(\mathcal{B})$ is fully faithful) the image of $\operatorname{Ho}\left(\operatorname{Ind}_{\kappa}(\mathcal{A})\right)$ is a localizing subcategory generated by a small set of objects. Applying the form of Neeman's Theorem proved by Krause in [46, 7.2.1] now implies that

$$
\operatorname{Ho}_{\operatorname{Ind}_{\kappa}}(\mathcal{B})^{\kappa} / \operatorname{Ho}_{\operatorname{Ind}_{\kappa}}(\mathcal{A})^{\kappa} \longrightarrow \operatorname{Ho}_{\operatorname{Ind}_{\kappa}}(\mathcal{B}) / \operatorname{Ho}_{\operatorname{Ind}_{\kappa}}(\mathcal{A})
$$

is a fully faithful map. Since [48, 1.4.4.1] implies that there is an equivalence $\operatorname{Ho}\left(\operatorname{Ind}_{\kappa}(\mathcal{A})^{\kappa}\right) \simeq \operatorname{Ho}\left(\operatorname{Ind}_{\kappa}(\mathcal{A})\right)^{\kappa}$ and $\operatorname{Ind}_{\kappa}(\mathcal{A})^{\kappa} \simeq \mathcal{A}$ up to idempotent completion (and similarly for $\mathcal{B}$ ), we conclude that the left vertical map is fully faithful.

Finally, this map is essentially surjective because there is a commutative (up to natural isomorphism) triangle

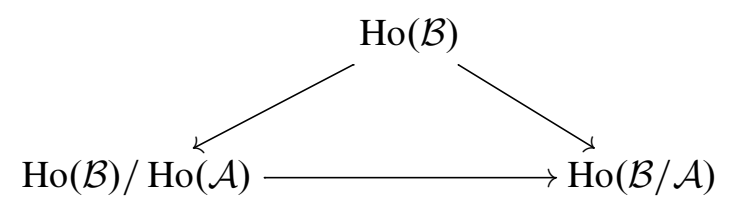

such that both maps from $\operatorname{Ho}(\mathcal{B})$ are essentially surjective.

Now we can obtain the following correspondence between exact sequences of small stable $\infty$-categories and exact sequences of triangulated categories.

Proposition 5.15 A sequence of $\kappa$-cocomplete small stable $\infty$-categories and $\kappa-$ small colimit preserving functors $\mathcal{A} \rightarrow \mathcal{B} \rightarrow \mathcal{C}$ is exact if and only if the associated sequence $\operatorname{Ho}(\mathcal{A}) \rightarrow \operatorname{Ho}(\mathcal{B}) \rightarrow \operatorname{Ho}(\mathcal{C})$ of triangulated categories is exact, in the sense that the composite is trivial, $\operatorname{Ho}(\mathcal{A}) \rightarrow \operatorname{Ho}(\mathcal{B})$ is fully faithful and the map $\operatorname{Ho}(\mathcal{B}) / \operatorname{Ho}(\mathcal{A}) \rightarrow \operatorname{Ho}(\mathcal{C})$ is an equivalence after idempotent completion. 
Proof Suppose $\mathcal{A} \rightarrow \mathcal{B} \rightarrow \mathcal{C}$ is exact. Then the composite is trivial, $\mathcal{A} \rightarrow \mathcal{B}$ is fully faithful and $\mathcal{B} / \mathcal{A} \rightarrow \mathcal{C}$ is an equivalence up to idempotent completion, and so the same must be true on the level of triangulated homotopy categories. Thus it is enough to show that $\operatorname{Ho}(\mathcal{B}) / \operatorname{Ho}(\mathcal{A}) \rightarrow \operatorname{Ho}(\mathcal{C})$ is an equivalence up to idempotent completion, which follows from Proposition 5.14. Conversely, suppose that

$$
\mathrm{Ho}(\mathcal{A}) \longrightarrow \mathrm{Ho}(\mathcal{B}) \longrightarrow \mathrm{Ho}(\mathcal{C})
$$

is exact. Then $\mathcal{A} \rightarrow \mathcal{B}$ is fully faithful by Proposition 5.10 , and the equivalences $\operatorname{Ho}(\mathcal{B} / \mathcal{A}) \simeq \operatorname{Ho}(\mathcal{B}) / \operatorname{Ho}(\mathcal{A}) \simeq \operatorname{Ho}(\mathcal{C})$ (the last up to idempotent completion) implies that $\mathcal{B} / \mathcal{A} \simeq \mathcal{C}$ by Corollary 5.11 .

Finally, we record a technical proposition that is used in the context of our construction of nonconnective $K$-theory. First, we need a technical lemma about the behavior of the Ind functor.

Lemma 5.16 Let $\mathcal{A} \rightarrow \mathcal{B}$ be an exact functor of small stable $\infty$-categories. Then the induced map $\operatorname{Ind}(\mathcal{A}) \rightarrow \operatorname{Ind}(\mathcal{B})$ of presentable stable $\infty$-categories preserves $\kappa$-compact objects for all infinite regular cardinals $\kappa$.

Proof Recall that a right adjoint preserves $\kappa$-filtered colimits if and only if its left adjoint preserves $\kappa$-compact objects [47, 5.5.1.4]. Since functors which preserve filtered colimits also preserve $\kappa$-filtered colimits and Ind( -$)$ takes exact functors to functors which preserve compact objects, the result follows.

Note that in the statement of the following proposition, we implicitly use the facts that stable $\infty$-categories have all finite colimits and exact functors preserve finite colimits.

Proposition 5.17 Let $\mathcal{A} \rightarrow \mathcal{B} \rightarrow \mathcal{C}$ be an exact sequence of small stable $\infty$-categories. Then for any infinite regular cardinal $\kappa$,

$$
\operatorname{Ind}(\mathcal{A})^{\kappa} \longrightarrow \operatorname{Ind}(\mathcal{B})^{\kappa} \longrightarrow \operatorname{Ind}(\mathcal{C})^{\kappa}
$$

is an exact sequence of idempotent-complete small stable $\infty$-categories.

Proof First, by Proposition 5.15, it suffices to check that

$$
\mathrm{Ho}\left(\operatorname{Ind}(\mathcal{A})^{\kappa}\right) \longrightarrow \mathrm{Ho}\left(\operatorname{Ind}(\mathcal{B})^{\kappa}\right) \longrightarrow \operatorname{Ho}\left(\operatorname{Ind}(\mathcal{C})^{\kappa}\right)
$$

is an exact sequence of triangulated categories. Again, we will deduce this from Neeman's generalization of Thomason's Localization Theorem (see [57, 4.4.9] or [56]), as follows. First, observe that $[48,1.4 .4 .1]$ implies that there is an equivalence 
$\operatorname{Ho}\left(\operatorname{Ind}(\mathcal{A})^{\kappa}\right) \simeq \operatorname{Ho}(\operatorname{Ind}(\mathcal{A}))^{\kappa}$ (and analogous equivalences for the other terms in the sequence). Next, since $\operatorname{Ind}(\mathcal{A})$ and $\operatorname{Ind}(\mathcal{B})$ are presentable, the criterion of [45] (characterizing well-generated triangulated categories) and [48, 1.4.4.2] imply that $\operatorname{Ho}(\operatorname{Ind}(\mathcal{B}))$ is well-generated and (since the map $\operatorname{Ind}(\mathcal{A}) \rightarrow \operatorname{Ind}(\mathcal{B})$ is fully faithful) the image of $\operatorname{Ho}(\operatorname{Ind}(\mathcal{A}))$ is a localizing subcategory generated by a small set of objects. Once again, the localization theorem $[46,7.2 .1]$ implies that

$$
\operatorname{Ho}(\operatorname{Ind}(\mathcal{B}))^{\kappa} / \operatorname{Ho}(\operatorname{Ind}(\mathcal{A}))^{\kappa} \longrightarrow \operatorname{Ho}(\operatorname{Ind}(\mathcal{B} / \mathcal{A}))^{\kappa}
$$

is an equivalence up to idempotent completion. The hypothesis that $\mathcal{B} / \mathcal{A} \rightarrow \mathcal{C}$ is an equivalence up to idempotent completion now implies the result.

\subsection{Split-exact sequences}

We will be particularly interested in exact sequences which are split in the following sense.

Definition 5.18 An exact sequence of small $\kappa$-cocomplete stable $\infty$-categories and $\kappa$-small colimit preserving functors

$$
\mathcal{A} \stackrel{f}{\longrightarrow} \mathcal{B} \stackrel{g}{\longrightarrow} \mathcal{C}
$$

is called split-exact if there exist exact functors $i: \mathcal{B} \rightarrow \mathcal{A}$ and $j: \mathcal{C} \rightarrow \mathcal{B}$, right adjoint to $f$ and $g$, respectively, such that $i \circ f \simeq \operatorname{Id}$ and $g \circ j \simeq$ Id via the adjunction morphisms.

We will also be interested in (split-)exact sequences of spectral categories.

Definition 5.19 A sequence $\mathcal{A} \rightarrow \mathcal{B} \rightarrow \mathcal{C}$ of spectral categories is exact if the induced sequence of stable presentable $\infty$-categories

$$
\mathrm{N}\left(\Omega^{\infty} \operatorname{Mod}(\mathcal{A})^{\mathrm{cf}}\right) \longrightarrow \mathrm{N}\left(\Omega^{\infty} \operatorname{Mod}(\mathcal{B})^{\mathrm{cf}}\right) \longrightarrow \mathrm{N}\left(\Omega^{\infty} \operatorname{Mod}(\mathcal{C})^{\mathrm{cf}}\right)
$$

is exact.

The following characterization is an immediate corollary of Proposition 5.15.

Proposition 5.20 A sequence $\mathcal{A} \rightarrow \mathcal{B} \rightarrow \mathcal{C}$ of spectral categories is exact if and only if the induced sequence of triangulated categories

$$
\mathcal{D}(\mathcal{A}) \longrightarrow \mathcal{D}(\mathcal{B}) \longrightarrow \mathcal{D}(\mathcal{C})
$$

is exact. 
Next, observe that we can relate these notions as follows (the proof of which is immediate):

Proposition 5.21 Let $\mathcal{A} \rightarrow \mathcal{B} \rightarrow \mathcal{C}$ be an (split-)exact sequence of small spectral categories. Then $\Psi_{\text {perf }}(\mathcal{A}) \rightarrow \Psi_{\text {perf }}(\mathcal{B}) \rightarrow \Psi_{\text {perf }}(\mathcal{C})$ is a (split-)exact sequence of small stable $\infty$-categories.

We also have an essential converse statement.

Proposition 5.22 Let $\mathcal{A} \rightarrow \mathcal{B} \rightarrow \mathcal{C}$ be a (split-)exact sequence of small stable $\infty-$ categories. Then there exists a (split-)exact sequence of small stable spectral categories

$$
\widetilde{\mathcal{A}} \longrightarrow \widetilde{\mathcal{B}} \longrightarrow \widetilde{\mathcal{C}}
$$

such that $\Psi_{\text {perf }}(\widetilde{\mathcal{A}} \rightarrow \widetilde{\mathcal{B}} \rightarrow \widetilde{\mathcal{C}})$ is naturally equivalent to $\mathcal{A} \rightarrow \mathcal{B} \rightarrow \mathcal{C}$.

Proof This follows from Proposition 4.28.

\subsection{Approximating split-exact sequences}

In order to localize with respect to the (split-)exact sequences, we need to be able to choose a set of representatives which generate them under filtered colimits.

Lemma 5.23 The full subcategory $\left(\mathrm{Cat}_{\infty}^{\mathrm{perf}}\right)^{\omega} \subset \mathrm{Cat}_{\infty}^{\mathrm{perf}}$ of compact small stable idempotent-complete $\infty$-categories is essentially small. More generally, for any regular cardinal $\kappa$, the category $\left(\mathrm{Cat}_{\infty}^{\mathrm{perf}}\right)^{\kappa}$ is essentially small.

Proof This is immediate from Corollary 4.25 and the fact that the full subcategory of $\kappa$-compact objects in a presentable $\infty$-category is essentially small.

This has the following immediate and essential corollary:

Corollary 5.24 For any regular cardinal $\kappa$, there exists a small set $\mathcal{E}$ of representatives of split-exact sequences of $\kappa$-compact small idempotent-complete stable $\infty$-categories.

It is straightforward to see that filtered colimits of exact sequences of such $\infty$-categories are exact.

Lemma 5.25 Given a filtered diagram of exact sequences $\mathcal{A}_{\alpha} \rightarrow \mathcal{B}_{\alpha} \rightarrow \mathcal{C}_{\alpha}$ of compact idempotent-complete small stable $\infty$-categories, the colimit $\mathcal{A} \rightarrow \mathcal{B} \rightarrow \mathcal{C}$ is an exact sequence of idempotent-complete small stable $\infty$-categories; that is, $\mathcal{A} \rightarrow \mathcal{B}$ is fully faithful with cofiber $\mathcal{C}$. 
Proof This follows from the fact that the filtered colimit of fully faithful functors is a fully faithful functor and that the cofiber of a filtered colimit of fully faithful functors is equivalent to the filtered colimit of the cofibers.

The $\infty$-category of $\infty$-categories equipped with a localization,

$$
\operatorname{Loc}\left(\operatorname{Cat}_{\infty}\right) \subseteq \operatorname{Fun}\left(\Delta^{1}, \operatorname{Cat}_{\infty}\right),
$$

is the subcategory of those functors $g: \mathcal{B} \rightarrow \mathcal{C}$ such that $g$ admits a right adjoint $j$ with $g \circ j \simeq \operatorname{Id}_{\mathcal{C}}$, and maps those commutative diagrams which also commute with the adjoint. We have obvious analogues $\operatorname{Loc}\left(\mathrm{Cat}_{\infty}^{\mathrm{ex}}\right)$ and $\operatorname{Loc}\left(\mathrm{Cat}_{\infty}^{\mathrm{perf}}\right)$, and in the stable setting a localization is part of the data of a split-exact sequence. We write

$$
\operatorname{Split}\left(\mathrm{Cat}_{\infty}^{\mathrm{ex}}\right) \subseteq \operatorname{Fun}\left(\Delta^{2}, \mathrm{Cat}_{\infty}^{\mathrm{ex}}\right)
$$

for the subcategory consisting of those diagrams $\mathcal{A} \stackrel{f}{\rightarrow} \mathcal{B} \stackrel{g}{\rightarrow} \mathcal{C}$ of small stable $\infty-$ categories such that $g \circ f \simeq 0, f$ is fully faithful with cofiber $g, f$ admits a right adjoint $i$ with $\operatorname{Id}_{\mathcal{A}} \simeq i \circ f$ and $g$ admits a right adjoint $j$ with $g \circ j \simeq \operatorname{Id}_{\mathcal{C}}$; maps are those commutative diagrams

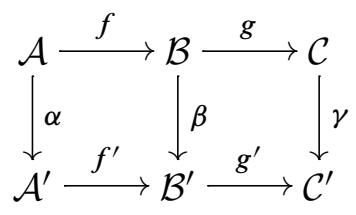

which also commute with the adjoints, ie $\alpha \circ i \simeq i^{\prime} \circ \beta$ and $\beta \circ j \simeq j^{\prime} \circ \gamma$.

Proposition 5.26 The functors

$$
\operatorname{Split}\left(\mathrm{Cat}_{\infty}^{\mathrm{ex}}\right) \longrightarrow \operatorname{Loc}\left(\mathrm{Cat}_{\infty}^{\mathrm{ex}}\right) \text { and } \operatorname{Split}\left(\mathrm{Cat}_{\infty}^{\mathrm{perf}}\right) \longrightarrow \operatorname{Loc}\left(\mathrm{Cat}_{\infty}^{\mathrm{perf}}\right),
$$

induced by the inclusion $\Delta^{1} \cong \Delta^{\{1,2\}} \rightarrow \Delta^{2}$, are equivalences.

Proof First observe that a split-exact sequence $\mathcal{A} \stackrel{f}{\rightarrow} \mathcal{B} \stackrel{g}{\rightarrow} \mathcal{C}$ is completely determined by the projection $g: \mathcal{B} \rightarrow \mathcal{C}$ together with its section $j: \mathcal{C} \rightarrow \mathcal{B}$. This is because $f: \mathcal{A} \rightarrow \mathcal{B}$ is the fiber of $g$, which we may identify with the full subcategory of $\mathcal{B}$ spanned by the $b \in \mathcal{B}$ such that $g(b) \simeq 0$, and, since $f$ is fully faithful, $i: \mathcal{B} \rightarrow \mathcal{A}$ is determined by the composite $f \circ i: \mathcal{B} \rightarrow \mathcal{A} \rightarrow \mathcal{B}$, the fiber

$$
f \circ i \longrightarrow \operatorname{id}_{\mathcal{B}} \longrightarrow j \circ g
$$

of the unit map of the adjunction $(g, j)$. Hence $\operatorname{Split}\left(\mathrm{Cat}_{\infty}^{\text {perf }}\right) \rightarrow \operatorname{Loc}\left(\mathrm{Cat}_{\infty}^{\text {perf }}\right)$ has contractible (homotopy) fibers and is therefore and equivalence. 
Proposition 5.27 The $\infty$-category Split $\left(\mathrm{Cat}_{\infty}^{\mathrm{perf}}\right)$ of split-exact sequences of small stable $\infty$-categories is accessible. In particular, there exists a cardinal $\kappa$ such that any split-exact sequence in $\mathrm{Cat}_{\infty}^{\mathrm{perf}}$ is a $\kappa$-filtered (and hence filtered) colimit of $\kappa$-compact split-exact sequences in $\mathrm{Cat}_{\infty}^{\mathrm{perf}}$.

Proof By Proposition 5.26, we may equivalently show that $\operatorname{Loc}\left(\mathrm{Cat}_{\infty}^{\text {perf }}\right)$ is accessible. Recall that an adjunction of $\infty$-categories can be described as a map $\mathcal{M} \rightarrow \Delta^{1}$ which is both a cocartesian fibration and a cartesian fibration [47, 5.2.2.1]. This leads us to consider the commutative diagram of pullback squares

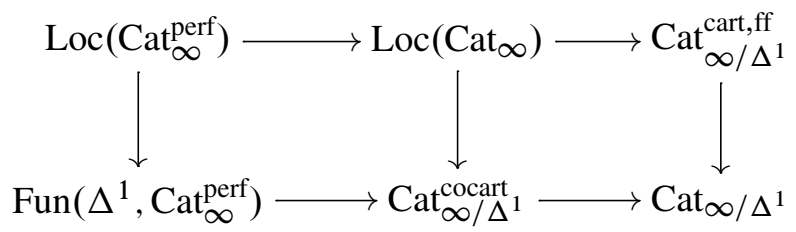

in which $\mathrm{Cat}_{\infty / \Delta^{1}}^{\text {cocart }} \subset \mathrm{Cat}_{\infty / \Delta^{1}}$ (respectively, Cat ${ }_{\infty / \Delta^{1}}^{\text {cart,ff }} \subset \mathrm{Cat}_{\infty / \Delta^{1}}$ ) denote the subcategories of cocartesian fibrations (respectively, cartesian fibrations whose straightenings are fully faithful) and functors which preserve cocartesian (respectively, cartesian) edges.

Since $\mathrm{Cat}_{\infty}^{\text {perf }} \subseteq \mathrm{Cat}_{\infty}$ is an accessible functor between accessible $\infty$-categories, it suffices, by [47, 5.4.4.3, 5.4.5.16, 5.4.6.6] and the duality between cartesian and cocartesian fibrations, to show that $\mathrm{Cat}_{\infty / \Delta^{1}}^{\text {cart,ff }}$ is accessible, and that the inclusions

$$
\mathrm{Cat}_{\infty / \Delta^{1}}^{\text {cart,ff }} \subseteq \mathrm{Cat}_{\infty / \Delta^{1}}^{\text {cart }} \subseteq \mathrm{Cat}_{\infty / \Delta^{1}}
$$

are accessible functors. The straightening functor gives an equivalence

$$
\mathrm{Cat}_{\infty / \Delta^{1}}^{\mathrm{cart}} \simeq \operatorname{Pre}_{\mathrm{Cat}_{\infty}}\left(\Delta^{1}\right)
$$

between cartesian fibrations over $\Delta^{1}$ and presheaves of $\infty$-categories on $\Delta^{1}[47$, 3.2.0.1].

In order to understand the condition of being fully faithful, we write $\mathrm{Cat}_{\infty}$ as an accessible localization $\mathrm{Cat}_{\infty} \subseteq \operatorname{Pre}(\mathrm{N}(\Delta))$ of simplicial spaces [43]. A functor is fully faithful when the corresponding map of (local) simplicial spaces is fully faithful, and recall that a map of simplicial spaces $j: X \rightarrow Y$ is fully faithful if and only if

$$
\operatorname{map}\left(\Delta^{1}, X\right) \longrightarrow \operatorname{map}\left(\partial \Delta^{1}, X\right) \times_{\operatorname{map}\left(\partial \Delta^{1}, Y\right)} \operatorname{map}\left(\Delta^{1}, Y\right)
$$

is an equivalence. Thus $\mathrm{Cat}_{\infty / \Delta^{1}}^{\text {cart,ff }}$ is the accessible localization of $\operatorname{Pre}\left(\Delta^{1} \times \mathrm{N}(\Delta)\right)$ obtained by also inverting the pushout product of $\operatorname{Id}_{\Delta^{1}}$ and $\partial \Delta^{1} \rightarrow \Delta^{1}$. It follows that

$$
\mathrm{Cat}_{\infty / \Delta^{1}}^{\text {cart,ff }} \text { and } \quad \mathrm{Cat}_{\infty / \Delta^{1}}^{\text {cart,ff }} \subseteq \mathrm{Cat}_{\infty / \Delta^{1}}^{\text {cart }}
$$


are accessible.

Finally, it remains to show that the inclusion $\mathrm{Cat}_{\infty / \Delta^{1}}^{\mathrm{cart}} \subseteq \mathrm{Cat}_{\infty / \Delta^{1}}$ is accessible. First, observe that finite limits commute with filtered colimits in $\mathrm{Cat}_{\infty}$, as $\mathrm{Cat}_{\infty} \simeq \operatorname{Ind}\left(\mathrm{Cat}_{\infty}^{\omega}\right)$ is compactly generated, the inclusion $\operatorname{Ind}\left(\mathrm{Cat}_{\infty}^{\omega}\right) \subseteq \operatorname{Pre}\left(\mathrm{Cat}_{\infty}^{\omega}\right)$ preserves limits and filtered colimits [47, 5.3.5.3], and finite limits commute with filtered colimits in presheaf $\infty$-categories (this uses $[47,5.3 .3 .3]$ and the fact that (co)limits in presheaf $\infty-$ categories are computed objectwise). It follows that the filtered colimit $\mathcal{C} \simeq \operatorname{colim}_{i} \mathcal{C}_{i}$ of cartesian fibrations $p_{i}: \mathcal{C}_{i} \rightarrow \Delta^{1}$, computed in Cat ${ }_{\infty}$, is itself a cartesian fibration $p: \mathcal{C} \rightarrow \Delta^{1}$; indeed, the inclusions $\mathcal{C}_{i} \rightarrow \mathcal{C}$ preserve cartesian edges over $\operatorname{Id}_{\Delta^{1}}$, and inspection of the fibers

$$
\mathcal{C} \times{ }_{\Delta^{1}} \Delta^{0} \simeq\left(\operatorname{colim} \mathcal{C}_{i}\right) \times{ }_{\Delta^{1}} \Delta^{0} \simeq \operatorname{colim}\left(\mathcal{C}_{i} \times{ }_{\Delta^{1}} \Delta^{0}\right)
$$

over each vertex $\Delta^{0} \rightarrow \Delta^{1}$ shows that $p: \mathcal{C} \rightarrow \Delta^{1}$ is also the colimit in $\mathrm{Cat}_{\infty / \Delta^{1}}^{\text {cart }}$.

\subsection{Strict-exact sequences}

Definition 5.28 An exact sequence of small stable $\infty$-categories of the form

$$
\mathcal{A} \longrightarrow \mathcal{B} \longrightarrow \mathcal{B} / \mathcal{A}
$$

is called strict-exact if $\mathcal{A} \rightarrow \mathcal{B}$ is the inclusion of a full subcategory and any object of $\mathcal{B}$ which is a summand of an object of $\mathcal{A}$ is also in $\mathcal{A}$. In particular, every split-exact sequence (see Definition 5.18) is equivalent to a strict-exact exact sequence.

We denote by $\underline{\mathcal{E}}_{\mathrm{wL}}^{\kappa}$ a small set of representatives of strict-exact sequences $\mathcal{A} \rightarrow \mathcal{B} \rightarrow \mathcal{B} / \mathcal{A}$ with $\mathcal{B}$ in $\left(\mathrm{Cat}_{\infty}^{\mathrm{ex}}\right)^{\kappa}$.

Proposition 5.30 Any strict-exact sequence $\mathcal{A} \rightarrow \mathcal{B} \rightarrow \mathcal{B} / \mathcal{A}$ is a $\kappa$-filtered colimit of strict-exact sequences $\mathcal{A}_{\alpha} \rightarrow \mathcal{B}_{\alpha} \rightarrow \mathcal{B}_{\alpha} / \mathcal{A}_{\alpha}$ in $\underline{\mathcal{E}}_{\mathrm{wL}}^{\kappa}$.

Proof Write $\mathcal{B} \simeq \operatorname{colim}_{\alpha} \mathcal{B}_{\alpha}$ as a $\kappa$-filtered colimit of $\kappa$-compact stable $\infty$-categories $\mathcal{B}_{\alpha}$, and define $\mathcal{A}_{\alpha}=\mathcal{A} \times_{\mathcal{B}} \mathcal{B}_{\alpha}$ to be the full subcategory of $\mathcal{A}$ consisting of those objects of $\mathcal{A}$ which lie in the image of $\mathcal{B}_{\alpha}$. Evidently, $\mathcal{A} \rightarrow \mathcal{B} \rightarrow \mathcal{B} / \mathcal{A}$ is the $\kappa$-filtered colimit of the exact sequences $\mathcal{A}_{\alpha} \rightarrow \mathcal{B}_{\alpha} \rightarrow \mathcal{B}_{\alpha} / \mathcal{A}_{\alpha}$, and $\mathcal{A}_{\alpha} \rightarrow \mathcal{B}_{\alpha} \rightarrow \mathcal{B}_{\alpha} / \mathcal{A}_{\alpha}$ is strict-exact because if $Y \in \mathcal{B}_{\alpha}$ is a summand of $X \in \mathcal{A}_{\alpha}$ then $Y \in \mathcal{A}_{\alpha}$ because the image of $Y$ in $\mathcal{B}$ lies in $\mathcal{A}$.

We denote by $\mathcal{E}_{\mathrm{L}}^{\kappa}$ a set of representatives of maps of the form $\mathcal{A} \rightarrow \operatorname{Idem}(\mathcal{A})$ with $\mathcal{A}$ in $\left(\mathrm{Cat}_{\infty}^{\mathrm{ex}}\right)^{\kappa}$. 
Proposition 5.31 Any map of the form $\mathcal{A} \rightarrow \operatorname{Idem}(\mathcal{A})$ is a $\kappa$-filtered colimit of elements of $\mathcal{E}_{\mathrm{L}}^{\kappa}$.

Proof Write $\mathcal{A} \simeq \operatorname{colim}_{\alpha} \mathcal{A}_{\alpha}$ as a $\kappa$-filtered colimit of $\kappa$-compact small stable $\infty$-categories $\mathcal{A}_{\alpha}$. Then $\operatorname{Idem}(\mathcal{A}) \simeq \operatorname{colim} \alpha \operatorname{Idem}\left(\mathcal{A}_{\alpha}\right)$, since Idem (viewed as an endofunctor of $\mathrm{Cat}_{\infty}^{\mathrm{ex}}$ ) commutes with $\kappa$-filtered colimits; this follows from the characterization of Idem in terms of a subcategory of the Ind category [47, 5.4.2.4] and the fact that filtered colimits in $\mathrm{Cat}_{\infty}^{\mathrm{ex}}$ can be computed in $\mathrm{Cat}_{\infty}[48,1.1 .4 .6]$.

\section{Additivity}

In this section we construct the universal additive invariant of small stable $\infty$-categories; see Theorem 6.10. Its construction is divided in two steps: First, using Proposition 5.27, we construct the unstable version; see Theorem 6.7. Then, by stabilizing, we obtain the universal additive invariant. Our arguments follow the pattern of the analogous result for dg-categories given in [68].

Definition 6.1 Let $\mathcal{D}$ be a stable presentable $\infty$-category. A functor

$$
E: \mathrm{Cat}_{\infty}^{\mathrm{ex}} \longrightarrow \mathcal{D}
$$

is called an additive invariant of small stable $\infty$-categories if it inverts Morita equivalences (see Definition 2.14), preserves filtered colimits and satisfies additivity, ie given a split-exact sequence

$$
\mathcal{A} \underset{i}{\stackrel{f}{\leftrightarrows}} \mathcal{C} \underset{j}{\stackrel{g}{\leftrightarrows}} \mathcal{B}
$$

the functors $i$ and $g$ induce an equivalence in $\mathcal{D}$,

$$
E(\mathcal{A}) \vee E(\mathcal{B}) \stackrel{\sim}{\longrightarrow} E(\mathcal{C}) .
$$

We denote by $\operatorname{Fun}_{\text {add }}\left(\mathrm{Cat}_{\infty}^{\mathrm{ex}}, \mathcal{D}\right)$ the $\infty$-category of additive invariants with values in $\mathcal{D}$.

Example 6.3 As we discuss in Sections 7 and 10, appropriate versions of algebraic $K$-theory and topological Hochschild homology provide additive invariants of small stable $\infty$-categories. 


\subsection{The unstable version}

Let us denote by $\operatorname{Pre}\left(\left(\mathrm{Cat}_{\infty}^{\mathrm{perf}}\right)^{\omega}\right)_{*}$ the $\infty$-category

$$
\operatorname{Fun}\left(\left(\left(\mathrm{Cat}_{\infty}^{\mathrm{perf}}\right)^{\omega}\right)^{\mathrm{op}}, \mathcal{T}_{\infty}\right)_{*}
$$

of presheaves of pointed spaces on the essentially small $\infty$-category $\left(\mathrm{Cat}_{\infty}^{\text {perf }}\right)^{\omega}$ of compact idempotent-complete small stable $\infty$-categories.

Lemma 6.4 Let $\mathcal{D}$ be a pointed presentable $\infty$-category. Then, we have an equivalence of $\infty$-categories

$$
\operatorname{Fun}^{\mathrm{L}}\left(\operatorname{Pre}\left(\left(\mathrm{Cat}_{\infty}^{\text {perf }}\right)^{\omega}\right)_{*}, \mathcal{D}\right) \simeq \operatorname{Fun}_{\mathrm{ftt}}\left(\mathrm{Cat}_{\infty}^{\text {perf }}, \mathcal{D}\right),
$$

where the right-hand side denotes the $\infty$-category of morphisms of $\infty$-categories which preserve filtered colimits.

Proof The proof is a consequence of the equivalences

$$
\begin{aligned}
\operatorname{Fun}^{\mathrm{L}}\left(\operatorname{Pre}\left(\left(\mathrm{Cat}_{\infty}^{\mathrm{perf}}\right)^{\omega}\right)_{*}, \mathcal{D}\right) \simeq \operatorname{Fun}\left(\left(\mathrm{Cat}_{\infty}^{\mathrm{perf}}\right)^{\omega}, \mathcal{D}\right) & \simeq \operatorname{Fun}_{\mathrm{ftt}}\left(\operatorname{Ind}\left(\left(\mathrm{Cat}_{\infty}^{\mathrm{perf}}\right)^{\omega}\right), \mathcal{D}\right) \\
& \simeq \operatorname{Fun}_{\mathrm{flt}}\left(\mathrm{Cat}_{\infty}^{\text {perf }}, \mathcal{D}\right),
\end{aligned}
$$

where the first follows from $[47,5.1 .5 .6]$ and the fact that $\mathcal{D}$ is pointed, and the last follows from Corollary 4.25 .

Let

$$
\phi: \mathrm{Cat}_{\infty}^{\text {perf }} \longrightarrow \operatorname{Pre}\left(\left(\mathrm{Cat}_{\infty}^{\text {perf }}\right)^{\omega}\right)_{*}
$$

be the functor obtained by first taking the Yoneda embedding and then restricting the presheaves to the category ( $\left.\mathrm{Cat}_{\infty}^{\text {perf }}\right)^{\omega}$. Recall from Corollary 5.24 that we can choose a fixed small set $\mathcal{E}$ of representatives of split-exact sequences in $\left(\mathrm{Cat}_{\infty}^{\mathrm{perf}}\right)^{\omega}$. We denote by $\mathcal{M}_{\text {add }}^{\text {un }}$ the localization of $\operatorname{Pre}\left(\left(\mathrm{Cat}_{\infty}^{\text {perf }}\right)^{\omega}\right)_{*}[47,5.5 .4 .15]$ with respect to the set of maps

$$
\phi(\mathcal{C}) / \phi(\mathcal{A}) \longrightarrow \phi(\mathcal{B})
$$

where $\mathcal{A} \rightarrow \mathcal{C} \rightarrow \mathcal{B}$ is a split-exact sequence in $\mathcal{E}$. Finally, let $\mathcal{U}_{\text {add }}^{\text {un }}$ be the composite

$$
\left.\mathrm{Cat}_{\infty}^{\mathrm{ex}} \stackrel{\text { Idem }(-)}{\longrightarrow} \mathrm{Cat}_{\infty}^{\mathrm{perf}} \stackrel{\phi}{\longrightarrow} \operatorname{Pre}\left(\mathrm{Cat}_{\infty}^{\mathrm{perf}}\right)^{\omega}\right)_{*} \stackrel{\gamma}{\longrightarrow} \mathcal{M}_{\mathrm{add}}^{\mathrm{un}},
$$

where $\gamma$ is the localization functor. 
Theorem 6.7 The functor $\mathcal{U}_{\text {add }}^{\text {un }}$ inverts Morita equivalences, preserves filtered colimits and sends split-exact sequences in Cat ${ }_{\infty}^{\mathrm{ex}}$ to cofiber sequences in $\mathcal{M}_{\mathrm{add}}^{\mathrm{un}}$. Moreover, $\mathcal{U}_{\text {add }}^{\text {un }}$ is universal with respect to these properties, ie given any pointed presentable $\infty$-category $\mathcal{D}$, we have an equivalence of $\infty$-categories

$$
\left(\mathcal{U}_{\text {add }}^{\text {un }}\right)^{*}: \operatorname{Fun}^{\mathrm{L}}\left(\mathcal{M}_{\text {add }}^{\text {un }}, \mathcal{D}\right) \stackrel{\sim}{\longrightarrow} \operatorname{Fun}_{\text {add }}^{\text {un }}\left(\operatorname{Cat}_{\infty}^{\text {ex }}, \mathcal{D}\right),
$$

where the right-hand denotes the full subcategory of Fun $\left(\mathrm{Cat}_{\infty}^{\mathrm{ex}}, \mathcal{D}\right)$ of morphisms of $\infty$-categories which satisfy the above conditions.

Proof The result follows from Definition 2.14, Lemma 6.4 and from the universal property of Bousfield localization (see Section 2.5: The functor $\phi$ preserves filtered colimits and by Proposition 5.27 any split-exact sequence can be approximated by a filtered colimit of split-exact sequences in $\mathcal{E}$ ).

\subsection{The universal additive invariant}

Let $\mathcal{M}_{\text {add }}$ be the stabilization $\operatorname{Stab}\left(\mathcal{M}_{\text {add }}^{\text {un }}\right)$ [48, Section 1.4] of $\mathcal{M}_{\text {add }}^{\text {un }}$; by construction, this is a stable $\infty$-category. Denote by $\mathcal{U}_{\text {add }}$ the following composite

$$
\mathrm{Cat}_{\infty}^{\mathrm{ex}} \stackrel{\mathcal{U}_{\text {add }}^{\text {un }}}{\longrightarrow} \mathcal{M}_{\mathrm{add}}^{\text {un }} \longrightarrow \operatorname{Stab}\left(\mathcal{M}_{\mathrm{add}}^{\text {un }}\right)
$$

Remark 6.8 Note that we have the equivalences

$$
\begin{aligned}
\operatorname{Stab}\left(\operatorname{Pre}\left(\left(\mathrm{Cat}_{\infty}^{\text {perf }}\right)^{\omega}\right)_{*}\right) & =\operatorname{Stab}\left(\operatorname{Fun}\left(\left(\left(\mathrm{Cat}_{\infty}^{\text {perf }}\right)^{\omega}\right)^{\mathrm{op}}, \mathcal{T}_{\infty *}\right)\right) \\
& \left.\simeq \operatorname{Fun}\left(\left(\left(\mathrm{Cat}_{\infty}^{\mathrm{perf}}\right)^{\omega}\right)^{\mathrm{op}}, \operatorname{Stab}\left(\mathcal{T}_{\infty *}\right)\right)\right) \\
& \simeq \operatorname{Fun}\left(\left(\left(\mathrm{Cat}_{\infty}^{\mathrm{perf}}\right)^{\omega}\right)^{\mathrm{op}}, \mathcal{S}_{\infty}\right),
\end{aligned}
$$

the last of which follows from [48, 1.4.4.11]. Therefore, defining

$$
\operatorname{Pre}_{\mathcal{S}_{\infty}}\left(\left(\mathrm{Cat}_{\infty}^{\text {perf }}\right)^{\omega}\right)=\operatorname{Fun}\left(\left(\left(\mathrm{Cat}_{\infty}^{\text {perf }}\right)^{\omega}\right)^{\mathrm{op}}, \mathcal{S}_{\infty}\right)
$$

and writing

$$
\psi: \mathrm{Cat}_{\infty}^{\text {perf }} \longrightarrow \operatorname{Pre}_{\mathcal{S}_{\infty}}\left(\left(\mathrm{Cat}_{\infty}^{\text {perf }}\right)^{\omega}\right)
$$

for the natural functor, we see that $\mathcal{M}_{\text {add }}$ can alternately be described as the localization of $\operatorname{Pre}_{\mathcal{S}_{\infty}}\left(\left(\mathrm{Cat}_{\infty}^{\text {perf }}\right)^{\omega}\right)$ with respect to the set of maps

$$
\psi(\mathcal{C}) / \psi(\mathcal{A}) \longrightarrow \psi(\mathcal{B})
$$

where $\mathcal{A} \rightarrow \mathcal{C} \rightarrow \mathcal{B}$ is a split-exact sequence in $\mathcal{E}$. 
Theorem 6.10 The functor $\mathcal{U}_{\text {add }}$ is the universal additive invariant, ie given any stable presentable $\infty$-category $\mathcal{D}$, we have an equivalence of $\infty$-categories

$$
\left(\mathcal{U}_{\text {add }}\right)^{*}: \operatorname{Fun}^{\mathrm{L}}\left(\mathcal{M}_{\text {add }}, \mathcal{D}\right) \stackrel{\sim}{\longrightarrow} \operatorname{Fun}_{\text {add }}\left(\mathrm{Cat}_{\infty}^{\mathrm{ex}}, \mathcal{D}\right) \text {. }
$$

Proof The result follows from Theorem 6.7 and from the universal property of stabilization (ie $[48,1.4 .4 .5])$. Note that stabilization preserves colimits and $\mathcal{U}_{\text {add }}^{\text {un }}$ sends split-exact sequences to cofiber sequences, so the split-exact sequence (6.2) is sent to a split cofiber sequence $\mathcal{U}_{\text {add }}(\mathcal{C}) \simeq \mathcal{U}_{\text {add }}(\mathcal{A}) \vee \mathcal{U}_{\text {add }}(\mathcal{B})$ in $\mathcal{M}_{\text {add }}$.

\section{Connective $K$-theory}

In this section, we verify that higher algebraic $K$-theory provides an additive invariant of small stable $\infty$-categories; Theorem 6.10 then applies to show that this invariant descends to $\mathcal{M}_{\text {add }}$. Furthermore, following the outline of [68], we prove the essential result that algebraic $K$-theory in fact becomes corepresentable in $\mathcal{M}_{\text {add }}$ (see Theorem 7.13). The underlying point is that Waldhausen's $S_{\bullet}$ construction simply becomes the suspension in $\mathcal{M}_{\text {add }}$. This result will allow us to understand transformations between additive theories from algebraic $K$-theory via the Yoneda Lemma; we use this in Section 10 to characterize the cyclotomic trace map. We begin by developing the necessary background on the construction of algebraic $K$-theory for small $\infty$-categories with finite colimits, and in Section 7.2 we compare the $K$-theory of a suitable Waldhausen category with the $K$-theory of its underlying $\infty$-category.

\subsection{Algebraic $K$-theory of $\infty$-categories}

Waldhausen's algebraic $K$-theory functor takes as input a category with cofibrations and weak equivalences. It is now well understood that, under mild hypotheses, the $K$-theory spectrum is determined by the Dwyer-Kan localization $L^{H} \mathcal{C}$ of the Waldhausen category $\mathcal{C}$; see Toën and Vezzosi [74], the first author and Mandell [12] and Cisinski [19]. Since $\mathrm{N}\left(\left(L^{H} \mathcal{C}\right)^{\text {fib }}\right)$ yields the $\infty$-category associated to $\mathcal{C}$, these results can be interpreted as saying that the algebraic $K$-theory of a Waldhausen category is an invariant of the underlying $\infty$-category. Moreover, it has long been folklore that given a sufficiently good theory of $\infty$-categories one can define analogues of Waldhausen's construction of algebraic $K$-theory (eg see [74, Section 7] for a sketch of such a definition in the context of Segal categories). In this subsection we study a version of such a direct construction of the algebraic $K$-theory of $\infty$-categories in the setting of quasicategories $[48,1.2 .2 .5]$. We prove that Waldhausen's algebraic 
$K$-theory of a Waldhausen category $\mathcal{C}$ is equivalent as a spectrum to this $\infty$-categorical algebraic $K$-theory of the associated $\infty$-category $\mathrm{N}\left(\left(L^{H} \mathcal{C}\right)^{\mathrm{fib}}\right)$.

We begin by reviewing Waldhausen's $S_{\bullet}$ construction. Let $\mathcal{C}$ be a Waldhausen category. Let $\operatorname{Ar}[n]$ denote the category of arrows in $[n]: \operatorname{Ar}[n]$ has objects $(i, j)$ for $0 \leq i \leq j \leq n$ and a unique map $(i, j) \rightarrow\left(i^{\prime}, j^{\prime}\right)$ for $i \leq i^{\prime}$ and $j \leq j^{\prime}$. Then $S_{n} \mathcal{C}$ is the full subcategory of the category of functors $A: \operatorname{Ar}[n] \rightarrow \mathcal{C}$ such that:

- $A_{i, i}=*$ for all $i$.

- The map $A_{i, j} \rightarrow A_{i, k}$ is a $w$-cofibration for all $i \leq j \leq k$.

- The diagram

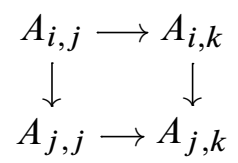

is a pushout square for all $i \leq j \leq k$.

The algebraic $K$-theory space of $\mathcal{C}$ is then defined to be $\Omega\left|w_{\bullet} S_{\bullet} \mathcal{C}\right|$, where the weak equivalences in $S_{\bullet} \mathcal{C}$ are defined pointwise. Furthermore, since each $S_{n} \mathcal{C}$ is itself a Waldhausen category (with the Reedy cofibrations), we can iterate the $S_{\bullet}$ construction. The algebraic $K$-theory spectrum of $\mathcal{C}$ is the spectrum with $n$-th space $\left|w_{\bullet} S_{\bullet}^{(n)} \mathcal{C}\right|$.

Now let $\mathcal{C}$ be a small pointed $\infty$-category with finite colimits. The following definition $[48,1.2 .2 .2]$ is the $\infty$-categorical analogue of Waldhausen's $S_{\bullet}$ construction.

Definition 7.1 Let $\operatorname{Gap}([n], \mathcal{C})$ be the full subcategory of $\operatorname{Fun}(\mathrm{N}(\operatorname{Ar}[n]), \mathcal{C})$ spanned by the functors $\mathrm{N}(\operatorname{Ar}[n]) \rightarrow \mathcal{C}$ such that, for each $i \in I, F(i, i)$ is a zero object of $\mathcal{C}$, and for each $i<j<k$, the square

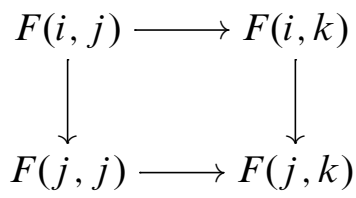

is cocartesian.

Remark 7.2 There is an obvious generalization of this definition to small pointed $\infty$-categories equipped with a suitable subcategory of "cofibrations" (satisfying the usual axioms, eg that cofibrations are stable under cobase change). However, in the presence of factorization hypotheses, this does not yield added generality; see [12, 1.3], which under such assumptions describes the $K$-theory space in terms of the Dwyer-Kan localization $\mathcal{C}$ regarded as a category with weak equivalences. 
As with the classical $S_{\bullet}$ construction, when $\mathcal{C}$ has all colimits, the data of the cocartesian squares (ie cofibers for the maps $F(i, j) \rightarrow F(i, k))$ is necessary only for the simplicial structure.

Lemma 7.3 Let $\mathcal{C}$ be an $\infty$-category with finite colimits. Then for each $n$, the forgetful functor

$$
\operatorname{Gap}([n], \mathcal{C}) \longrightarrow \operatorname{Fun}\left(\Delta^{\{1,2, \ldots, n\}}, \mathcal{C}\right)
$$

is an equivalence of $\infty$-categories (and observe that $\Delta^{\{1,2, \ldots, n\}} \simeq \mathrm{N}([n-1])$ ).

Proof This follows from the fact that the space of colimits for a given diagram in an $\infty$-category is contractible $[47,1.2 .12 .9,1.2 .13 .5]$. Alternatively, a constructive proof along the lines of $[11,2.9]$ (using a mapping cylinder argument) can be given using the comparison discussed in Section 7.2 below.

Remark 7.4 Lemma 7.3 implies that $\operatorname{Gap}([n], \mathcal{C})$ is stable when $\mathcal{C}$ is stable.

Following [48, 1.2.2.5], we define a simplicial $\infty$-category $S_{\bullet}^{\infty} \mathcal{C}$ by the rule $S_{n}^{\infty} \mathcal{C}=$ $\operatorname{Gap}([n], \mathcal{C})$. Applying passage to the largest Kan complex levelwise, we obtain a simplicial space $\left(S_{\bullet}^{\infty} \mathcal{C}\right)_{\text {iso }}$. Then $\Omega\left|\left(S_{\bullet}^{\infty} \mathcal{C}\right)_{\text {iso }}\right|$ is the $\infty$-categorical version of Waldhausen's $K$-theory space. Furthermore, for each $n, \operatorname{Gap}([n], \mathcal{C})$ is itself a small pointed $\infty$-category with finite colimits: once again, we can iterate this procedure. Since $\operatorname{Gap}([0], \mathcal{C})$ is contractible (with preferred basepoint given by the point in $\mathcal{C}$ ) and $\operatorname{Gap}([1], \mathcal{C})$ is equivalent to $\mathcal{C}$, there is a natural map

$$
S^{1} \wedge(\mathcal{C})_{\text {iso }} \longrightarrow\left|\left(S_{\bullet}^{\infty} \mathcal{C}\right)_{\text {iso }}\right|
$$

given by the inclusion into the 1 -skeleton. Therefore, the spaces $\left|\left(\left(S_{\bullet}^{\infty}\right)^{n}(\mathcal{C})\right)_{\text {iso }}\right|$ assemble to form a spectrum $K(\mathcal{C})$; this is the $\infty$-categorical version of Waldhausen's $K$-theory spectrum. We can see from the definition that $\operatorname{Gap}([n], \mathcal{C})$ is natural in (right) exact functors, and therefore $S_{\bullet}^{\infty} \mathcal{C}$ and $\left|\left(S_{\bullet}^{\infty} \mathcal{C}\right)_{\text {iso }}\right|$ are also natural. Since the equivalence $\operatorname{Gap}([1], \mathcal{C}) \rightarrow \operatorname{Fun}(*, \mathcal{C}) \simeq \mathcal{C}$ induced by the restriction map is natural in $\mathcal{C}$, we deduce that the $K$-theory spectrum is natural in exact functors.

In practice, we find it more convenient to use an "all at once" reformulation of the definition of the iterated $S_{\bullet}$ construction (eg see [12, A.5.4], [13, 2.2], the appendix to [32] and also [62, Section 2]).

Definition 7.5 Write $\operatorname{Ar}_{n_{1}, \ldots, n_{q}}$ for $\operatorname{Ar}\left[n_{1}\right] \times \cdots \times \operatorname{Ar}\left[n_{q}\right]$. For a functor

$$
A: \mathrm{N}\left(\operatorname{Ar}_{n_{1}, \ldots, n_{q}}\right)=\mathrm{N}\left(\operatorname{Ar}\left[n_{1}\right] \times \cdots \times \operatorname{Ar}\left[n_{q}\right]\right) \longrightarrow \mathcal{C},
$$


we write $A_{i_{1}, j_{1} ; \ldots ; i_{q}, j_{q}}$ for the value of $A$ on the object $\left(\left(i_{1}, j_{1}\right), \ldots,\left(i_{q}, j_{q}\right)\right)$. Let $\operatorname{Gap}\left(\left(\left[n_{1}\right], \ldots,\left[n_{q}\right]\right), \mathcal{C}\right)$ be the full subcategory of $\operatorname{Fun}\left(\mathrm{N}\left(\operatorname{Ar}_{n_{1}, \ldots, n_{q}}\right), \mathcal{C}\right)$ spanned by the functors such that:

- $A_{i_{1}, j_{1} ; \ldots ; i_{q}, j_{q}} \simeq *$ whenever $i_{k}=j_{k}$ for some $k$.

- For every object $\left(i_{1}, j_{1} ; \ldots ; i_{q}, j_{q}\right)$ in $\operatorname{Ar}\left[n_{1}\right] \times \cdots \times \operatorname{Ar}\left[n_{q}\right]$, every $1 \leq r \leq q$, and every $j_{r} \leq k \leq n_{r}$, the square

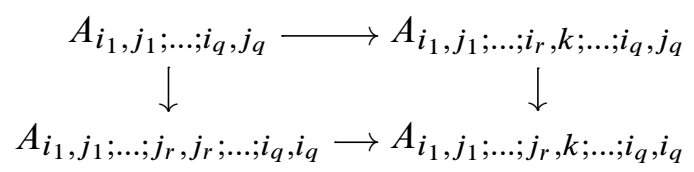

is a cocartesian square.

Now we define the multisimplicial $\infty$-category

$$
\left(S^{\infty}\right)_{n_{1}, \ldots, n_{q}}^{(q)} \mathcal{C}=\operatorname{Gap}\left(\left(\left[n_{1}\right], \ldots,\left[n_{q}\right]\right), \mathcal{C}\right)
$$

We regard $\left(S_{\bullet}^{\infty}\right)^{(0)}$ as $\mathcal{C}$ and it is clear that $\left(S_{\bullet}^{\infty}\right)_{n}^{(1)}$ is $\operatorname{Gap}([n], \mathcal{C})$. Now we directly the define the $K$-theory spectrum of an $\infty$-category $\mathcal{C}$ with finite colimits to be the spectrum with $q$-th space

$$
K \mathcal{C}(q)=\left(\left(S^{\infty}\right)_{\bullet, \ldots, \bullet}^{(q)}\right)_{\text {iso }},
$$

The suspension maps $\Sigma K \mathcal{C}(q) \rightarrow K(q+1)$ are induced on diagrams by the projection map

$$
\operatorname{Ar}\left[n_{1}\right] \times \cdots \times \operatorname{Ar}\left[n_{q}\right] \times \operatorname{Ar}\left[n_{q+1}\right] \longrightarrow \operatorname{Ar}\left[n_{1}\right] \times \cdots \times \operatorname{Ar}\left[n_{q}\right]
$$

From Definition 7.5 it is now clear that the construction of the $K$-theory spectrum is functorial in (right) exact functors.

\subsection{Comparison with Waldhausen's $K$-theory}

We now establish a comparison between Waldhausen's algebraic $K$-theory of a Waldhausen category $\mathcal{C}$ and the $\infty$-categorical version of the algebraic $K$-theory of the associated simplicial category $L^{H} \mathcal{C}$. The comparison is essentially a consequence of the theory of rigidification of homotopy coherent diagrams to strict diagrams in a model category (originally studied by Dwyer-Kan [29]), which allows us to pass between $\infty-$ categorical diagrams and point-set diagrams, and the "homotopical" $S_{\bullet}^{\prime}$ construction of [11], which allows us to replace the use of pushouts by homotopy pushouts for suitable Waldhausen categories. The version of the comparison of homotopy coherent diagrams to strict diagrams we use is originally due to Hirschowitz-Simpson [40] in the context 
of Segal categories (see also Rezk's work in Segal spaces [60, 8.12]). Since we are using quasicategories in this paper, we work with the version proved by Lurie in that setting [47, 4.2.4.4].

Let $S$ be a small simplicial set, $\mathcal{D}$ a small simplicial category, and $u: \mathfrak{C}[S] \rightarrow \mathcal{D}$ an equivalence. Let $\mathcal{A}$ be a combinatorial simplicial model category, and let $\mathcal{U}$ be a $\mathcal{D}$-chunk of $\mathcal{A}$ (see [47, A.3.4.9] for a discussion of $\mathcal{D}$-chunks). Then the induced map

$$
\mathrm{N}\left(\left(\mathcal{U}^{\mathcal{D}}\right)^{\mathrm{cf}}\right) \longrightarrow \operatorname{Fun}\left(S, \mathrm{~N}\left(\mathcal{U}^{\mathrm{cf}}\right)\right)
$$

is a categorical equivalence of simplicial sets. Here the notation $\left(\mathcal{U}^{\mathcal{D}}\right)^{\text {cf }}$ indicates the full subcategory of $\mathcal{A}^{\mathcal{D}}$ consisting of cofibrant-fibrant objects (in the projective model structure) landing in $\mathcal{U}$.

Specializing to our situation, assume that $S$ is the (ordinary) nerve $\mathrm{N}(J)$ of a diagram (small category) $J$; that is, $J$ is regarded as a discrete simplicial category. Then the counit map $\mathfrak{C}[\mathrm{N}(J)] \rightarrow J$ is an equivalence and so we have that the induced map

$$
\mathrm{N}\left(\left(\mathcal{U}^{J}\right)^{\mathrm{cf}}\right) \longrightarrow \operatorname{Fun}\left(\mathrm{N}(J), \mathrm{N}\left(\mathcal{U}^{\mathrm{cf}}\right)\right)
$$

is a categorical equivalence of simplicial sets.

Lemma 7.6 Let $\mathcal{A}$ be a combinatorial simplicial model category and $\mathcal{C} \subset \mathcal{A}$ a full subcategory. Then for each $n$ the induced map

$$
\mathrm{N}\left(\left(\mathcal{C}^{\mathrm{Ar}[n]}\right)^{\mathrm{cf}}\right) \longrightarrow \operatorname{Fun}\left(\mathrm{N}(\operatorname{Ar}[n]), \mathrm{N}\left(\mathcal{C}^{\mathrm{cf}}\right)\right)
$$

is a categorical equivalence of simplicial sets.

Proof By [47, A.3.4.15], we can choose a small subcategory $\mathcal{V} \subset \mathcal{A}$ which contains $\mathcal{C}$ and such that $\mathcal{V}$ is an $(\operatorname{Ar}[n])$-chunk for each $n$ and moreover $\mathrm{N}\left((\mathcal{C})^{\text {cf }}\right)$ is equivalent to $\mathrm{N}\left((\mathcal{V})^{\mathrm{cf}}\right)$. Then as discussed above, [47, 4.2.4.4] implies that for each $n$ the natural map

$$
\mathrm{N}\left(\left((\mathcal{V})^{\mathrm{Ar}[n]}\right)^{\mathrm{cf}}\right) \longrightarrow \operatorname{Fun}\left(\mathrm{N}(\operatorname{Ar}[n]), \mathrm{N}\left((\mathcal{V})^{\mathrm{cf}}\right)\right)
$$

is a categorical equivalence of simplicial sets.

To apply these rigidification results, we use the $S_{\bullet}^{\prime}$ construction. The $S_{\bullet}^{\prime}$ construction $[11,2.7]$ is a variant of Waldhausen's $S_{\bullet}$ construction defined by replacing the cocartesian squares in the definition of $S$. with homotopy cocartesian squares. In order to define the $S_{\bullet}^{\prime}$ construction, we must work with Waldhausen categories for which there is a reasonable notion of homotopy cocartesian squares. We briefly recall this theory from [11, Section 2]. A map is a weak cofibration if it is equivalent by a zig-zag to a 
cofibration, and a square is a homotopy cocartesian square if it equivalent by a zig-zag to a pushout square with one leg a cofibration. For control on these notions, we require the hypothesis that any map in $\mathcal{C}$ can be factored (not necessarily functorially) as a cofibration followed by a weak equivalence.

For such a Waldhausen category $\mathcal{C}$, we can then define $S_{n}^{\prime} \mathcal{C}$ to be the full subcategory of the category of functors $\operatorname{Ar}[n] \rightarrow \mathcal{C}$ such that:

- $A_{i, i} \simeq *$ for all $i$.

- The map $A_{i, j} \longrightarrow A_{i, k}$ is a weak cofibration for all $i \leq j \leq k$.

- The diagram

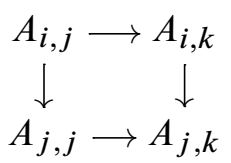

is a homotopy cocartesian square for all $i \leq j \leq k$.

By construction, the $S_{\bullet}^{\prime}$ construction is functorial in weakly exact functors, ie functors that preserve weak equivalences and homotopy cocartesian squares. Moreover, the natural inclusion

$$
w_{\bullet} S_{\bullet} \mathcal{C} \longrightarrow w_{\bullet} S_{\bullet}^{\prime} \mathcal{C}
$$

is a weak equivalence $[11,2.9]$. Therefore, we can equivalently define the algebraic $K$-theory space of a Waldhausen category $\mathcal{C}$ as $\Omega\left|w_{\bullet} S_{\bullet}^{\prime} \mathcal{C}\right|$ and similarly the algebraic $K$-theory spectrum of $\mathcal{C}$ as having $n$-th space $\left|w_{\bullet}\left(S_{\bullet}^{\prime}\right)^{(n)} \mathcal{C}\right|$.

Now, let $\mathcal{C}$ be a Waldhausen category that arises as a subcategory of a model category. Since a square is homotopy cocartesian in $\mathcal{C}$ if and only if it is a pushout square in $\mathrm{N}\left((\mathcal{C})^{\mathrm{cf}}\right)$, in this setting the equivalence of Lemma 7.6 restricts to give an equivalence

$$
\mathrm{N}\left(\left(S_{n}^{\prime} \mathcal{C}\right)^{\mathrm{cf}}\right) \longrightarrow \operatorname{Gap}\left([n], \mathrm{N}\left((\mathcal{C})^{\mathrm{cf}}\right)\right)
$$

Similar considerations for the iterated $S_{\bullet}^{\prime}$ construction [12, A.5.4] (as modeled in Definition 7.5) yield the equivalence

$$
\mathrm{N}\left(\left(S_{n_{1}, \ldots, n_{q}}^{\prime(q)} \mathcal{C}\right)^{\mathrm{cf}}\right) \longrightarrow \operatorname{Gap}\left(\left(\left[n_{1}\right], \ldots,\left[n_{q}\right]\right), \mathcal{C}\right) .
$$

Applying Proposition 2.10, we then obtain the following comparison of algebraic $K$-theory spaces and spectra. 
Corollary 7.7 Let $\mathcal{A}$ be a simplicial model category and $\mathcal{C} \subset \mathcal{A}$ a small full subcategory which has all finite homotopy colimits. Then for each $n$ there is a weak equivalence of simplicial sets

$$
\left|w_{\bullet} S_{n}^{\prime} \mathcal{C}\right| \simeq\left|\left(S_{n}^{\infty} \mathrm{N}\left((\mathcal{C})^{\mathrm{cf}}\right)\right)_{\text {iso }}\right| .
$$

and for each $\left(n_{1}, \ldots, n_{q}\right)$ there is a weak equivalence of simplicial sets

$$
\left|w_{\bullet} S_{n_{1}, \ldots, n_{q}}^{\prime(q)} \mathcal{C}\right| \simeq\left|\left(\left(S^{\infty}\right)_{n_{1}, \ldots, n_{q}}^{(q)} \mathrm{N}\left((\mathcal{C})^{\mathrm{cf}}\right)\right)_{\text {iso }}\right| .
$$

In particular, this yields the following theorem:

Theorem 7.8 Let $\mathcal{A}$ be a simplicial model category and $\mathcal{C} \subset \mathcal{A}$ a small full subcategory of the cofibrants which admits all homotopy pushouts and is a Waldhausen category via the model structure on $\mathcal{A}$. Then there is an equivalence of spectra

$$
K(\mathcal{C}) \simeq K\left(\mathrm{~N}\left((\mathcal{C})^{\mathrm{cf}}\right)\right)
$$

which is natural in weakly exact functors.

Finally, specializing to our case, we find the following result.

Corollary 7.9 Let $\mathcal{C}$ be a small pretriangulated spectral category and let $\mathcal{M}_{\mathcal{C}}$ denote the category of perfect $\mathcal{C}$-modules with its Waldhausen structure induced by the model structure on $\mathcal{C}$-modules. Then there is an isomorphism in the stable category

$$
K\left(\mathcal{M}_{\mathcal{C}}\right) \simeq K\left(\Psi_{\text {perf }} \mathcal{C}\right)
$$

As a consequence, Waldhausen's additivity theorem applies to prove the following proposition.

Proposition 7.10 The algebraic $K$-theory functor

$$
K: \mathrm{Cat}_{\infty}^{\text {perf }} \longrightarrow \mathcal{S}_{\infty}
$$

is an additive invariant.

Proof By Lemma 7.3, $K$ preserves equivalences of stable $\infty$-categories. Therefore, it suffices to show that $K$ preserves filtered colimits and split-exact sequences. The former follows from the fact that the $S_{\bullet}^{\infty}$ construction and restriction to the maximal subgroup preserve filtered colimits, as $\mathrm{N}(\operatorname{Ar}([n]))$ and $\Delta^{0}$ are compact $\infty$-categories. Corollary 7.9 allows us to reduce to consideration of split-exact sequences of spectral categories

$$
\widehat{\mathcal{A}}_{\text {perf }} \longrightarrow \widehat{\mathcal{C}}_{\text {perf }} \longrightarrow \widehat{\mathcal{B}}_{\text {perf }}
$$


As in [70], we observe that this sequence is Morita equivalent to the sequence

$$
\widehat{\mathcal{A}}_{\text {perf }} \longrightarrow E\left(\widehat{\mathcal{A}}_{\text {perf }}, \widehat{\mathcal{C}}_{\text {perf }}, \widehat{\mathcal{B}}_{\text {perf }}\right) \longrightarrow \widehat{\mathcal{B}}_{\text {perf }}
$$

(where $E$ denotes Waldhausen's category of cofiber sequences in $\mathcal{C}$ with first term in the image of $\mathcal{A}$ and cofiber in the image of $\mathcal{B}$ ). Now Waldhausen's additivity theorem implies the desired splitting on $K$-theory.

So far, all of our comparison results assume that the Waldhausen category we are working with arises as a subcategory of a model category. In fact, we can extend our comparison and functoriality results to Waldhausen categories $\mathcal{C}$ such that all maps admit (not necessarily functorial) factorizations as cofibrations followed by weak equivalences and which are DHKS-saturated (ie such that a map $f$ is a weak equivalence in $\mathcal{C}$ if and only if its image in the homotopy category is an isomorphism). We do this as follows, using a construction due to Cisinski [19, Section 4].

Lemma 7.11 Let $\mathcal{C}$ be a Waldhausen category with factorization and weak equivalences that are DHKS-saturated. Then there exists a Waldhausen category $\mathcal{M}(\mathcal{C})$ and a DK-equivalence $\mathcal{C} \rightarrow \mathcal{M}(\mathcal{C})$ which is natural in weakly exact functors.

Proof Given a Waldhausen category $\mathcal{C}$, let $\mathcal{P}(\mathcal{C})$ denote here the pointed simplicial presheaves on $\mathcal{C}$ with the projective model structure (ie weak equivalences and fibrations are determined pointwise). We can successively localize $\mathcal{P}(\mathcal{C})$ to produce a category of presheaves which are pointwise Kan complexes, preserve weak equivalences and take homotopy cocartesian squares in $\mathcal{C}$ to homotopy pullback squares in $\mathcal{P}(\mathcal{C})$; denote this category by $\mathcal{P}_{\mathrm{ex}}(\mathcal{C})[19,4.10]$. Let $\mathcal{M}(\mathcal{C})$ denote the full subcategory of the localized category consisting of the objects which are cofibrant and weakly equivalent to representable presheaves; this can be regarded as a Waldhausen category, inheriting structure from the model structure on $\mathcal{P}_{\text {ex }}(\mathcal{C})$. The Yoneda embedding induces a DKequivalence $\mathcal{C} \rightarrow \mathcal{M}(\mathcal{C})$ [19, 4.11], and a weakly exact functor $\mathcal{C} \rightarrow \mathcal{C}^{\prime}$ induces a left Quillen functor $\mathcal{P}_{\text {ex }}(\mathcal{C}) \rightarrow \mathcal{P}_{\text {ex }}\left(\mathcal{C}^{\prime}\right)$ by left Kan extension, and hence an exact functor $\mathcal{M}(\mathcal{C}) \rightarrow \mathcal{M}\left(\mathcal{C}^{\prime}\right)$ by restriction.

As a corollary, we have the following result comparing of the $K$-theory of Waldhausen categories that are DHKS-saturated and admit factorization to the associated $K$-theory of $\infty$-categories.

Corollary 7.12 In the setting of 7.11, there are equivalences

$$
K(\mathcal{C}) \longrightarrow K(\mathcal{M}(\mathcal{C})) \longrightarrow K\left(\mathrm{~N}\left((\mathcal{M}(\mathcal{C}))^{\mathrm{cf}}\right)\right)
$$

which are natural in weakly exact functors. 
Proof First, since we have a natural DK-equivalence $\mathcal{C} \rightarrow \mathcal{M}(\mathcal{C})$, there is a natural equivalence $K(\mathcal{C}) \rightarrow K(\mathcal{M}(\mathcal{C}))$; see [12], [19] and [74]. Next, since the category $\mathcal{M}(\mathcal{C})$ satisfies the hypothesis of Theorem 7.8, the second equivalence holds.

Given a (homotopically) pointed simplicial category with finite homotopy colimits, we can use essentially the same construction to produce a DK-equivalent Waldhausen category; see [74, Section 5] and [14, Section 14] for versions of such a construction.

\subsection{Corepresentability}

This subsection is entirely devoted to the proof of Theorem 7.13. The proof will follow from Propositions 7.17 and 7.19.

Theorem 7.13 Let $\mathcal{A}$ be a small stable $\infty$-category and $\mathcal{B}$ be a compact idempotentcomplete small stable $\infty$-category. Then there is a natural equivalence of spectra

$$
\operatorname{Map}\left(\mathcal{U}_{\text {add }}(\mathcal{B}), \mathcal{U}_{\text {add }}(\mathcal{A})\right) \simeq K\left(\operatorname{Fun}^{\mathrm{ex}}(\mathcal{B}, \operatorname{Idem}(\mathcal{A}))\right) .
$$

When $\mathcal{B}$ is the small stable $\infty$-category $\mathcal{S}_{\infty}^{\omega}$ of compact spectra, there is a natural equivalence of spectra

$$
\operatorname{Map}\left(\mathcal{U}_{\text {add }}\left(\mathcal{S}_{\infty}^{\omega}\right), \mathcal{U}_{\text {add }}(\mathcal{A})\right) \simeq K(\operatorname{Idem}(\mathcal{A}))
$$

In particular, we have isomorphisms of abelian groups

$$
\operatorname{Hom}\left(\mathcal{U}_{\text {add }}\left(\mathcal{S}_{\infty}^{\omega}\right), \Sigma^{-n} \mathcal{U}_{\text {add }}(\mathcal{A})\right) \simeq K_{n}(\operatorname{Idem}(\mathcal{A}))
$$

in the triangulated category $\operatorname{Ho}\left(\mathcal{M}_{\mathrm{add}}\right)$.

Notation 7.14 Given a small stable $\infty$-category $\mathcal{A}$, we denote by $K_{\mathcal{A}}^{w}$ the object

$$
\mathcal{B} \longmapsto\left|\left(S_{\bullet}^{\infty}\left(\operatorname{Fun}^{\mathrm{ex}}(\mathcal{B}, \operatorname{Idem}(\mathcal{A}))\right)\right)_{\text {iso }}\right|
$$

in $\operatorname{Pre}\left(\left(\mathrm{Cat}_{\infty}^{\text {perf }}\right)^{\omega}\right)_{*}$ and by $K_{\mathcal{A}}$ the object

$$
\mathcal{B} \longmapsto K\left(\operatorname{Fun}^{\text {ex }}(\mathcal{B}, \operatorname{Idem}(\mathcal{A}))\right)
$$

in $\operatorname{Pre}_{\mathcal{S}_{\infty}}\left(\left(\mathrm{Cat}_{\infty}^{\text {perf }}\right)^{\omega}\right)$. Note that the value of $K_{\mathcal{A}}$ at $\mathcal{S}_{\infty}^{\omega}$ is precisely the $K$-theory spectrum $K(\mathcal{A})$ of $\mathcal{A}$, and similarly that $K_{\mathcal{A}}^{w}$ is the delooping of the $K$-theory space.

Remark 7.15 Recall that Corollary 4.27 allows us to model the small $\infty$-category of exact functors $\operatorname{Fun}^{\operatorname{ex}}(\mathcal{B}, \operatorname{Idem}(\mathcal{A}))$ as the pretriangulated spectral category $\operatorname{rep}(\mathcal{B}, \mathcal{A})$ of right-compact $\Upsilon(\mathcal{A})^{\mathrm{op}} \wedge \Upsilon(\mathcal{B})$-modules. Combined with Proposition 2.10, this implies that the associated mapping space $\left(\operatorname{Fun}^{\mathrm{ex}}(\mathcal{B}, \operatorname{Idem}(\mathcal{A}))\right)_{\text {iso }}$ can be calculated 
as $\left|w_{\bullet} \operatorname{rep}(\mathcal{B}, \operatorname{Idem}(\mathcal{A}))\right|$. Moreover, $\operatorname{rep}(\mathcal{B}, \operatorname{Idem}(\mathcal{A}))$ inherits a natural Waldhausen structure as a full subcategory of the cofibrant objects in the model structure on the category of $\mathcal{B}$ - $\operatorname{Idem}(\mathcal{A})$-bimodules. As such, we can also consider the algebraic $K$-theory space $\left|w_{\bullet} S_{\bullet} \operatorname{rep}(\mathcal{B}, \operatorname{Idem}(\mathcal{A}))\right|$ and associated spectrum.

In the following results, we will use the observation that Waldhausen's $S_{\bullet}$ construction, applied to a spectral category which is a Waldhausen category with the cofibrations inherited from a spectral model structure with all objects fibrant, produces a spectral category (where the mapping spectra are given by an appropriate end) [10, Section 3]. To ensure we are in this setting, we will tacitly use the equivalent model of spectral categories enriched in EKMM $S$-modules, as explained in [14, Section 15]. Alternatively, we could stay with spectral categories in symmetric spectra and use the "Moore" $S$. construction from [10, Section 4], which uses an explicit model of the homotopy end. We also need the following lemma which allows us to bring the $S_{\bullet}$ construction inside:

Lemma 7.16 Let $\mathcal{A}$ and $\mathcal{B}$ be small stable $\infty$-categories. Then we have an equivalence of simplicial $\infty$-categories

$$
S_{\bullet}^{\infty} \operatorname{Fun}^{\mathrm{ex}}(\mathcal{B}, \mathcal{A}) \simeq \operatorname{Fun}^{\mathrm{ex}}\left(\mathcal{B}, S_{\bullet}^{\infty} \mathcal{A}\right)
$$

and correspondingly an equivalence of spaces

$$
\left|\left(S_{\bullet}^{\infty} \operatorname{Fun}^{\mathrm{ex}}(\mathcal{B}, \mathcal{A})\right)_{\text {iso }}\right| \simeq\left|\left(\operatorname{Fun}^{\mathrm{ex}}\left(\mathcal{B}, S_{\bullet}^{\infty} \mathcal{A}\right)\right)_{\text {iso }}\right| .
$$

Proof First, we show that for each $n$ there is an equivalence of $\infty$-categories

$$
\operatorname{Gap}\left([n], \operatorname{Fun}^{\mathrm{ex}}(\mathcal{B}, \mathcal{A})\right) \simeq \operatorname{Fun}^{\mathrm{ex}}(\mathcal{B}, \operatorname{Gap}([n], \mathcal{A})) .
$$

Since Fun $(-,-)$ is defined simply as the mapping simplicial set $[47,1.2 .7 .2]$, we have the equivalence

$$
\operatorname{Fun}(\mathrm{N}(\operatorname{Ar}[n]), \operatorname{Fun}(\mathcal{B}, \mathcal{A})) \simeq \operatorname{Fun}(\mathcal{B}, \operatorname{Fun}(\mathrm{N}(\operatorname{Ar}[n]), \mathcal{A})) .
$$

Since colimits in functor $\infty$-categories are computed pointwise [47, Section 5.1.2.3] and the $\infty$-category $\operatorname{Fun}^{\mathrm{ex}}(\mathcal{B}, \mathcal{A})$ is the full subcategory of $\operatorname{Fun}(\mathcal{B}, \mathcal{A})$ spanned by the exact functors, we have a map

$$
\operatorname{Gap}\left([n], \operatorname{Fun}^{\mathrm{ex}}(\mathcal{B}, \mathcal{A})\right) \longrightarrow \operatorname{Fun}^{\mathrm{ex}}(\mathcal{B}, \operatorname{Gap}([n], \mathcal{A})),
$$

and Lemma 7.3 implies that it is an equivalence. It is now straightforward to check that these comparison maps assemble into the desired simplicial equivalence.

We can now relate $\mathcal{M}_{\text {add }}$ to the algebraic $K$-theory presheaf. 
Proposition 7.17 Let $\mathcal{A}$ be a small stable $\infty$-category. Then, we have a natural equivalence $\Sigma\left(\mathcal{U}_{\text {add }}^{\text {un }}(\mathcal{A})\right) \simeq K_{\mathcal{A}}^{w}$ in $\mathcal{M}_{\text {add }}^{\text {un }}$ (see notation (6.6)) and a natural equivalence $\Sigma \mathcal{U}_{\text {add }}(\mathcal{A}) \simeq \Sigma K_{\mathcal{A}}$ in $\mathcal{M}_{\text {add }}$.

Proof We begin by handling the unstable case. Theorem 4.23 implies that we can model $\mathcal{A}$ by a small spectral category (which we still denote by $\mathcal{A}$ ). Following [51, 3.3], we consider the following sequence of simplicial spectral categories:

$$
\mathcal{A}_{\bullet} \stackrel{I}{\longrightarrow} P S_{\bullet} \mathcal{A} \stackrel{Q}{\longrightarrow} S_{\bullet} \mathcal{A},
$$

where $\mathcal{A}_{\bullet}$ is a constant simplicial object and $P S_{\bullet} \mathcal{A}$ is the simplicial path object of $S . \mathcal{A}$. By applying the functor $\mathcal{U}_{\text {add }}^{\text {un }}$ to this sequence, we obtain an induced morphism

$$
\Theta: \mathcal{U}_{\mathrm{add}}^{\mathrm{un}}\left(P S_{\bullet}^{\infty} \mathcal{A}\right) / \mathcal{U}_{\mathrm{add}}^{\mathrm{un}}\left(\mathcal{A}_{\bullet}\right) \longrightarrow \mathcal{U}_{\mathrm{add}}^{\mathrm{un}}\left(S_{\bullet}^{\infty} \mathcal{A}\right)
$$

of simplicial objects in $\mathcal{M}_{\text {add }}^{\text {un }}$. We now show that each component $\Theta_{n}$ of $\Theta$ is an equivalence. For each $n \geq 0$, we have a split-exact sequence

$$
\mathcal{A} \underset{I_{n}}{\stackrel{R_{n}}{\leftrightarrows}} P S_{n} \mathcal{A}=S_{n+1} \mathcal{A} \underset{Q_{n}}{\stackrel{S_{n}}{\leftrightarrows}} S_{n} \mathcal{A}
$$

in which

$$
\begin{aligned}
& I_{n}(A)=(* \rightarrow A \stackrel{\mathrm{Id}}{\longrightarrow} A \stackrel{\mathrm{Id}}{\longrightarrow} \cdots \stackrel{\mathrm{Id}}{\longrightarrow} A), \\
& Q_{n}\left(* \rightarrow A_{0} \rightarrow A_{1} \rightarrow \cdots \rightarrow A_{n}\right)=\left(A_{1} / A_{0} \rightarrow \cdots \rightarrow A_{n} / A_{0}\right), \\
& S_{n}\left(* \rightarrow A_{0} \rightarrow A_{1} \rightarrow \cdots \rightarrow A_{n-1}\right)=\left(* \rightarrow * \rightarrow A_{0} \rightarrow \cdots \rightarrow A_{n-1}\right), \\
& R_{n}\left(* \rightarrow A_{0} \rightarrow A_{1} \rightarrow \cdots \rightarrow A_{n-1}\right)=A_{0} .
\end{aligned}
$$

By the construction of $\mathcal{M}_{\text {add }}^{\text {un }}$ (and of $\mathcal{U}_{\text {add }}^{\text {un }}$ ), we conclude that the induced morphisms

$$
\Theta_{n}: \mathcal{U}_{\mathrm{add}}^{\mathrm{un}}\left(P S_{n}^{\infty} \mathcal{A}\right) / \mathcal{U}_{\mathrm{add}}^{\text {un }}(\mathcal{A}) \longrightarrow \mathcal{U}_{\mathrm{add}}^{\text {un }}\left(S_{n}^{\infty} \mathcal{A}\right), \quad n \geq 0,
$$

are equivalences in $\mathcal{M}_{\text {add }}^{\text {un }}$. This allow us to obtain the following cocartesian square:

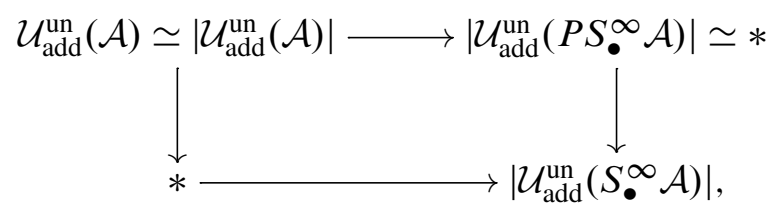

and so a natural equivalence

$$
\Sigma\left(\mathcal{U}_{\text {add }}^{\text {un }}(\mathcal{A})\right) \stackrel{\sim}{\longrightarrow}\left|\mathcal{U}_{\text {add }}^{\text {un }}\left(S_{\bullet}^{\infty} \mathcal{A}\right)\right|
$$


in $\mathcal{M}_{\text {add }}^{\text {un }}$. By combining this equivalence with the equivalences

$$
\begin{aligned}
\mathcal{U}_{\mathrm{add}}^{\mathrm{un}}\left(S_{\bullet}^{\infty} \mathcal{A}\right) & =\left|\left(\operatorname{Fun}^{\mathrm{ex}}\left(-, \operatorname{Idem}\left(S_{\bullet}^{\infty} \mathcal{A}\right)\right)\right)_{\text {iso }}\right| \\
& \simeq\left|\left(S_{\bullet}^{\infty} \operatorname{Fun}^{\mathrm{ex}}(-, \operatorname{Idem}(\mathcal{A}))\right)_{\text {iso }}\right| \\
& =K^{w}(\mathcal{A}),
\end{aligned}
$$

where (7.18) follows from Lemma 7.16, we conclude that $\Sigma\left(\mathcal{U}_{\text {add }}^{\text {un }}(\mathcal{A})\right) \simeq K_{\mathcal{A}}^{w}$ in $\mathcal{M}_{\text {add }}^{\text {un }}$. The identification in the stable setting follows from the unstable considerations and the usual passage from results on the $K$-theory space to the $K$-theory spectrum.

Proposition 7.19 Let $\mathcal{A}$ be a small stable $\infty$-category. Then, the presheaves $K_{\mathcal{A}}^{w}$ and $K_{\mathcal{A}}$ (see Notation 7.14) are local, ie given any split-exact sequence $\mathcal{B} \rightarrow \mathcal{C} \rightarrow \mathcal{D}$ in $\mathcal{E}$, the induced maps of spectra (see (6.5) and (6.9))

$$
\begin{aligned}
& \operatorname{map}\left(\phi(\mathcal{D}), K_{\mathcal{A}}^{w}\right) \stackrel{\sim}{\longrightarrow} \operatorname{Map}\left(\phi(\mathcal{C}) / \phi(\mathcal{A}), K_{\mathcal{A}}^{w}\right), \\
& \operatorname{map}\left(\psi(\mathcal{D}), K_{\mathcal{A}}\right) \stackrel{\sim}{\longrightarrow} \operatorname{Map}\left(\psi(\mathcal{C}) / \psi(\mathcal{A}), K_{\mathcal{A}}\right),
\end{aligned}
$$

are equivalences.

Proof The argument is exactly the same in both cases. Therefore, we discuss only the stable $K_{\mathcal{A}}$. Since $\mathcal{B}, \mathcal{C}$ and $\mathcal{D}$ belong to $\left(\mathrm{Cat}_{\infty}^{\text {perf }}\right)^{\omega}$, the spectral Yoneda Lemma shows us that we need to prove that the induced sequence of spectra

$$
K\left(\operatorname{Fun}^{\mathrm{ex}}(\mathcal{D}, \operatorname{Idem}(\mathcal{A}))\right) \longrightarrow K\left(\operatorname{Fun}^{\mathrm{ex}}(\mathcal{C}, \operatorname{Idem}(\mathcal{A}))\right) \longrightarrow K\left(\operatorname{Fun}^{\mathrm{ex}}(\mathcal{B}, \operatorname{Idem}(\mathcal{A}))\right)
$$

is a cofiber sequence. Using Corollary 4.27 it suffices to consider the split-exact sequence of small spectral categories

$$
\operatorname{rep}(\mathcal{D}, \mathcal{A}) \leftrightarrows \operatorname{rep}(\mathcal{C}, \mathcal{A}) \leftrightarrows \operatorname{rep}(\mathcal{B}, \mathcal{A})
$$

Note that, again by Corollary 4.27, all of these spectral categories carry a natural Waldhausen structure inherited from the usual model structure on spectral modules. We will apply Waldhausen's Fibration Theorem [78, 1.6.4]. We have the Waldhausen category $\operatorname{vrep}(\mathcal{C}, \mathcal{A})$, whose weak equivalences are the morphisms $f$ such that $\operatorname{Cone}(f)$ is contractible, as well as the Waldhausen category $w \operatorname{rep}(\mathcal{C}, \mathcal{A})$, with the same cofibrations as $v \operatorname{rep}(\mathcal{C}, \mathcal{A})$ but whose weak equivalences are those $f$ such that $\operatorname{Cone}(f)$ belongs to $\operatorname{rep}(\mathcal{D}, \mathcal{A})$. Moreover, we have a natural inclusion $\operatorname{vrep}(\mathcal{C}, \mathcal{A}) \subset w \operatorname{rep}(\mathcal{C}, \mathcal{A})$ and an equivalence $\operatorname{rep}(\mathcal{C}, \mathcal{A})^{w} \simeq \operatorname{rep}(\mathcal{D}, \mathcal{A}) ;$ see $[78, \S 1.6]$. The conditions of $[78,1.6 .4]$ are satisfied, so we obtain a cofiber sequence of spectra

$$
K(\operatorname{rep}(\mathcal{D}, \mathcal{A})) \longrightarrow K(\operatorname{rep}(\mathcal{C}, \mathcal{A})) \longrightarrow K(\operatorname{rep}(\mathcal{B}, \mathcal{A})) .
$$


Proof of Theorem 7.13 Let $\mathcal{A}$ be stable $\infty$-category and $\mathcal{B}$ a compact small idempotent-complete stable $\infty$-category. By Proposition 7.17 we have an equivalence $\mathcal{U}_{\text {add }}(\mathcal{A}) \simeq K_{\mathcal{A}}$ and by Proposition $7.19 K_{\mathcal{A}}$ is local. Therefore, we have the following natural equivalence:

$$
\operatorname{Map}\left(\mathcal{U}_{\text {add }}(\mathcal{B}), \mathcal{U}_{\text {add }}(\mathcal{A})\right) \simeq \operatorname{Map}\left(\psi(\mathcal{B}), K_{\mathcal{A}}\right),
$$

where the right-hand side is calculated in $\operatorname{Pre}\left(\left(\mathrm{Cat}_{\infty}^{\mathrm{perf}}\right)^{\omega} ; \mathcal{S}_{\infty}\right)$. Since $\mathcal{B}$ belongs to $\left(\mathrm{Cat}_{\infty}^{\text {perf }}\right)^{\omega}$, the presheaf $\psi(\mathcal{B})$ is representable and so by the spectral Yoneda Lemma we have $\operatorname{Map}\left(\psi(\mathcal{B}), K_{\mathcal{A}}\right) \simeq K_{\mathcal{A}}(\mathcal{B})$. Finally, since by definition of $K_{\mathcal{A}}$ we have $K_{\mathcal{A}}(\mathcal{B})=K\left(\operatorname{Fun}^{\mathrm{ex}}(\mathcal{B}, \operatorname{Idem}(\mathcal{A}))\right)$ the proof is finished.

\section{Localization}

The definition of additivity we study in this paper is given in terms of the condition that algebraic $K$-theory takes models of split-exact sequences of triangulated categories to (homotopy) cofiber sequences of spectra. This perspective is motivated in part by Neeman's reformulation of the Thomason-Trobaugh Localization Theorem [56]. Neeman observed that following Thomason-Trobaugh and using the construction of Bousfield localization, one could regard algebraic $K$-theory as in fact taking exact sequences of triangulated categories to cofiber sequences of spectra, provided one worked with nonconnective $K$-theory (see Theorem 9.34 for a version of this result). We will refer to such a theory as satisfying localization. In this section we construct the universal localizing invariant of small stable $\infty$-categories; see Theorem 8.7. Our work follows the general pattern of the analogous result for $\mathrm{dg}$-categories in [21].

Definition 8.1 Let $\mathcal{D}$ be a stable presentable $\infty$-category. A functor

$$
E: \mathrm{Cat}_{\infty}^{\mathrm{ex}} \longrightarrow \mathcal{D}
$$

is called a localizing invariant of small stable $\infty$-categories if it inverts Morita equivalences (see Definition 2.14), preserves filtered colimits and satisfies localization, ie sends exact sequences

$$
\mathcal{A} \longrightarrow \mathcal{B} \longrightarrow \mathcal{C}
$$

of small stable $\infty$-categories (see Definition 5.12) to cofiber sequences

$$
E(\mathcal{A}) \longrightarrow E(\mathcal{B}) \longrightarrow E(\mathcal{C})
$$

in $\mathcal{D}$. We denote by $\operatorname{Fun}_{\text {loc }}\left(\mathrm{Cat}_{\infty}^{\mathrm{ex}}, \mathcal{D}\right)$ the $\infty$-category of localizing invariants with values in $\mathcal{D}$. 
Every localizing invariant is an additive invariant (see Definition 6.1), since a split-exact sequence is exact. The converse does not hold, however: the impetus for the definition of nonconnective $K$-theory was precisely the fact that the connective algebraic $K$-theory functor does not satisfy localization. As we discuss in Sections 9 and 10, nonconnective algebraic $\mathrm{K}$-theory $(\boldsymbol{K})$ and topological Hochschild homology $(\mathrm{THH})$ are localizing invariants.

Although the universal localizing invariant can be constructed by direct localization, as in Section 6, we use a more involved procedure:

(i) First, we construct a variant of the universal additive invariant; see Proposition 8.3. We work with a general infinite regular cardinal $\kappa$, and we do not factor through $\mathrm{Cat}_{\infty}^{\text {perf }}$; that is, Morita equivalences are not inverted. This produces the functor

$$
\underline{\mathcal{U}}_{\mathrm{add}}^{\kappa}: \mathrm{Cat}_{\infty}^{\mathrm{ex}} \longrightarrow \underline{\mathcal{M}}_{\mathrm{add}}^{\kappa} .
$$

(ii) We localize $\underline{\mathcal{M}}_{\text {add }}^{\kappa}$ so that the exact sequences

$$
\mathcal{A} \longrightarrow \mathcal{B} \longrightarrow \mathcal{B} / \mathcal{A}
$$

with $\operatorname{Ho}(\mathcal{A})$ a thick triangulated subcategory of $\operatorname{Ho}(\mathcal{B})$, are sent to cofiber sequences; see Proposition 8.5. We then obtain the functor

$$
\underline{\mathcal{U}}_{\text {wloc }}^{\kappa}: \mathrm{Cat}_{\infty}^{\mathrm{ex}} \longrightarrow \underline{\mathcal{M}}_{\mathrm{wloc}}^{\kappa}
$$

(iii) We perform a localization of $\mathcal{M}_{\text {wloc }}^{\kappa}$ to force Morita equivalences to be sent to isomorphisms; see Proposition 8.6. We obtain then the functor

$$
\mathcal{U}_{\mathrm{loc}}^{\kappa}: \mathrm{Cat}_{\infty}^{\mathrm{ex}} \longrightarrow \mathcal{M}_{\mathrm{loc}}^{\kappa} .
$$

(iv) Finally, we localize $\mathcal{M}_{\text {loc }}^{\kappa}$ so that the functor $\mathcal{U}_{\text {loc }}^{\kappa}$ preserves filtered colimits; see Theorem 8.7. We end up with the universal localizing invariant

$$
\mathcal{U}_{\mathrm{loc}}: \mathrm{Cat}_{\infty}^{\mathrm{ex}} \longrightarrow \mathcal{M}_{\mathrm{loc}}
$$

The point of this seemingly circuitous process is that it enables a clear, conceptual proof of the corepresentability of nonconnective $K$-theory in $\mathcal{M}_{\text {loc }}$; see Section 9.

Notation 8.2 From now on and until the end of Section 9 we will work with a fixed infinite regular cardinal $\kappa$ larger than $\omega$, which we assume is sufficiently large so that Proposition 5.27 applies. We will denote by $\left(\mathrm{Cat}_{\infty}^{\mathrm{ex}}\right)^{\kappa}$ the category of $\kappa$-compact small stable $\infty$-categories (see Section 2.4). 


\subsection{Additive $\kappa$-variant}

Let

$$
\psi: \mathrm{Cat}_{\infty}^{\text {perf }} \longrightarrow \operatorname{Pre}\left(\left(\mathrm{Cat}_{\infty}^{\text {perf }}\right)^{\kappa} ; \mathcal{S}_{\infty}\right)
$$

be the functor obtained by first taking the Yoneda embedding and then restricting the presheaves to the $\infty$-category $\left(\mathrm{Cat}_{\infty}^{\mathrm{ex}}\right)^{\kappa}$. Corollary 5.24 allow us to choose a fixed small set $\underline{\mathcal{E}}_{\mathrm{A}}^{\kappa}$ of representatives of split-exact sequences in $\left(\mathrm{Cat}_{\infty}^{\mathrm{perf}}\right)^{\kappa}$. We denote by $\underline{\mathcal{M}}_{\text {add }}^{\kappa}$ the localization of $\operatorname{Pre}\left(\left(\mathrm{Cat}_{\infty}^{\mathrm{perf}}\right)^{\kappa} ; \mathcal{S}_{\infty}\right)$ with respect to the set of maps

$$
\operatorname{Cone}(\psi(\mathcal{A}) \longrightarrow \psi(\mathcal{C})) \longrightarrow \psi(\mathcal{B}),
$$

where $\mathcal{A} \rightarrow \mathcal{C} \rightarrow \mathcal{B}$ is a split-exact sequence in $\underline{\mathcal{E}}_{\mathrm{A}}^{\kappa}$. Let $\underline{\mathcal{U}}_{\text {add }}^{\kappa}$ be the following composite:

$$
\mathrm{Cat}_{\infty}^{\text {perf }} \stackrel{\psi}{\longrightarrow} \operatorname{Pre}\left(\left(\mathrm{Cat}_{\infty}^{\mathrm{perf}}\right)^{\kappa} ; \mathcal{S}_{\infty}\right) \stackrel{\gamma}{\longrightarrow} \underline{\mathcal{M}}_{\mathrm{add}}^{\kappa},
$$

where $\gamma$ is the localization functor.

Proposition 8.3 The functor $\underline{\mathcal{U}}_{\text {add }}^{\kappa}$ preserves $\kappa$-filtered colimits and sends split-exact sequences

$$
\mathcal{A} \underset{i}{\stackrel{f}{\leftrightarrows}} \mathcal{C} \underset{j}{\stackrel{g}{\leftrightarrows}} \mathcal{B}
$$

in $\mathrm{Cat}{ }_{\infty}^{\text {perf }}$ to (split) cofiber sequences in $\underline{\mathcal{M}}_{\text {add }}^{\kappa}$. Moreover, $\underline{\mathcal{U}}_{\text {add }}^{\kappa}$ is universal with respect to these two properties, ie given any stable presentable $\infty$-category $\mathcal{D}$, we have an equivalence of $\infty$-categories

$$
\left(\mathcal{U}_{\text {add }}^{\kappa}\right)^{*}: \operatorname{Fun}^{\mathrm{L}}\left(\mathcal{M}_{\text {add }}^{\kappa}, \mathcal{D}\right) \stackrel{\sim}{\longrightarrow} \operatorname{Fun}_{\underline{\text { add }}}^{\kappa}\left(\operatorname{Cat}_{\infty}^{\text {perf }}, \mathcal{D}\right),
$$

where the right-hand side denotes the full subcategory of Fun $\left(\mathrm{Cat}_{\infty}^{\mathrm{ex}}, \mathcal{D}\right)$ of morphisms of $\infty$-categories which satisfy the above two conditions.

Proof The result follows from the analogue of the argument for Lemma 6.4 in the context of $\kappa$-compact objects and $\operatorname{Ind}_{\kappa}$, and from the universal property of Bousfield localization (see Section 2.5). Specifically, the functor $\psi$ preserves $\kappa$-filtered colimits and Proposition 5.27 shows that any split-exact sequence can be approximated by a $\kappa$-filtered colimit of split-exact sequences in $\underline{\mathcal{E}}_{\mathrm{A}}^{\kappa}$.

Next, we localize $\underline{\mathcal{M}}_{\text {add }}^{\kappa}$ with respect to the set of maps

$$
\operatorname{Cone}\left(\underline{\mathcal{U}}_{\text {add }}^{\kappa}(\mathcal{A}) \longrightarrow \underline{\mathcal{U}}_{\text {add }}^{\kappa}(\mathcal{B})\right) \longrightarrow \underline{\mathcal{U}}_{\text {add }}^{\kappa}(\mathcal{B} / \mathcal{A}),
$$


where $\mathcal{A} \rightarrow \mathcal{B} \rightarrow \mathcal{B} / \mathcal{A}$ is a strict-exact sequence in $\underline{\mathcal{E}}_{\mathrm{wL}}^{\kappa}$ (see Section 5.5). Let $\underline{\mathcal{U}}_{\text {wloc }}^{\kappa}$ be the following composite:

$$
\mathrm{Cat}_{\infty}^{\mathrm{perf}} \stackrel{\underline{\mathcal{U}}_{\text {add }}^{\kappa}}{\longrightarrow} \underline{\mathcal{M}}_{\mathrm{add}}^{\kappa} \stackrel{\gamma}{\longrightarrow} \underline{\mathcal{M}}_{\mathrm{wloc}}^{\kappa}
$$

where $\gamma$ is the localization functor.

Proposition 8.5 The functor $\underline{\mathcal{U}}_{\text {wloc }}^{\kappa}$ preserves $\kappa$-filtered colimits and sends strict-exact sequences to cofiber sequences in $\underline{\mathcal{M}}_{\text {wloc }}^{\kappa}$ :

$$
\mathcal{A} \longrightarrow \mathcal{B} \longrightarrow \mathcal{B} / \mathcal{A} \longmapsto \underline{\mathcal{U}}_{\text {wloc }}^{\kappa}(\mathcal{A}) \longrightarrow \underline{\mathcal{U}}_{\mathrm{wloc}}^{\kappa}(\mathcal{B}) \longrightarrow \underline{\mathcal{U}}_{\text {wloc }}^{\kappa}(\mathcal{B} / \mathcal{A})
$$

Moreover, $\underline{\mathcal{U}}_{\text {wloc }}^{\kappa}$ is universal with respect to these two properties, ie given any stable presentable $\infty$-category $\mathcal{D}$, we have an equivalence of $\infty$-categories

$$
\left(\underline{\mathcal{U}}_{\text {wloc }}^{\kappa}\right)^{*}: \operatorname{Fun}^{\mathrm{L}}\left(\underline{\mathcal{M}}_{\text {wloc }}^{\kappa}, \mathcal{D}\right) \stackrel{\sim}{\longrightarrow} \operatorname{Fun}_{\underline{\text { wloc }}}^{\kappa}\left(\operatorname{Cat}_{\infty}^{\text {perf }}, \mathcal{D}\right),
$$

where the right-hand side denotes the full subcategory of Fun $\left(\mathrm{Cat}_{\infty}^{\mathrm{perf}}, \mathcal{D}\right)$ of morphisms of $\infty$-categories which satisfy the above two conditions.

Proof The result follows from Propositions 8.3 and 5.30 and from the universal property of localization (see Section 2.5).

\subsection{Morita equivalences}

We now localize $\underline{\mathcal{M}}_{\text {wloc }}^{\kappa}$ with respect to the set of maps

$$
\underline{\mathcal{U}}_{\text {wloc }}^{\kappa}(\mathcal{A} \longrightarrow \operatorname{Idem}(\mathcal{A})),
$$

where $\mathcal{A} \rightarrow \operatorname{Idem}(\mathcal{A})$ belongs to $\mathcal{E}_{\mathrm{L}}^{\kappa}$. Let $\mathcal{U}_{\text {loc }}^{\kappa}$ be the following composition:

$$
\mathrm{Cat}_{\infty}^{\mathrm{ex}} \stackrel{\underline{\mathcal{U}}_{\text {wloc }}^{\kappa}}{\longrightarrow} \underline{\mathcal{M}}_{\mathrm{wloc}}^{\kappa} \stackrel{\gamma}{\longrightarrow} \mathcal{M}_{\mathrm{loc}}^{\kappa},
$$

where $\gamma$ is the localization functor.

Proposition 8.6 The functor $\mathcal{U}_{\mathrm{loc}}^{\kappa}$ inverts Morita equivalences, preserves $\kappa$-filtered colimits and sends exact sequences $\mathcal{A} \rightarrow \mathcal{B} \rightarrow \mathcal{C}$ in $\mathrm{Cat}_{\infty}^{\mathrm{ex}}$ to cofiber sequences $\mathcal{U}_{\text {loc }}^{\kappa}(\mathcal{A}) \rightarrow \mathcal{U}_{\text {loc }}^{\kappa}(\mathcal{B}) \rightarrow \mathcal{U}_{\text {loc }}^{\kappa}(\mathcal{C})$ to cofiber sequences in $\mathcal{M}_{\mathrm{loc}}^{\kappa}$. Moreover, $\mathcal{U}_{\mathrm{loc}}^{\kappa}$ is universal with respect to these two properties, ie given any stable presentable $\infty-$ category $\mathcal{D}$, we have an equivalence of $\infty$-categories

$$
\left(\mathcal{U}_{\mathrm{loc}}^{\kappa}\right)^{*}: \operatorname{Fun}^{\mathrm{L}}\left(\mathcal{M}_{\mathrm{loc}}^{\kappa}, \mathcal{D}\right) \stackrel{\sim}{\longrightarrow} \operatorname{Fun}_{\mathrm{loc}}^{\kappa}\left(\mathrm{Cat}_{\infty}^{\mathrm{ex}}, \mathcal{D}\right)
$$

where the right-hand side denotes the full subcategory of Fun $\left(\mathrm{Cat}_{\infty}^{\mathrm{ex}}, \mathcal{D}\right)$ of morphisms of $\infty$-categories which satisfy the above three conditions. 
Proof The fact that $\mathcal{U}_{\mathrm{loc}}^{\kappa}$ preserves $\kappa$-filtered colimits is clear. Since a functor $\mathcal{A} \rightarrow \mathcal{B}$ is a Morita equivalence if and only if $\operatorname{Idem}(\mathcal{A}) \rightarrow \operatorname{Idem}(\mathcal{B})$ is an equivalence, Proposition 5.31 allow us to conclude that $\mathcal{U}_{\text {loc }}^{\kappa}$ inverts Morita equivalences. We now show that $\mathcal{U}_{\text {loc }}^{\kappa}$ sends exact sequences to cofiber sequences. Let

$$
\mathcal{A} \longrightarrow \mathcal{B} \longrightarrow \mathcal{C}
$$

be an exact sequence. Since we have an induced Morita equivalence $\mathcal{B} / \mathcal{A} \rightarrow \mathcal{C}$, it suffices to show that $\mathcal{U}_{\text {loc }}^{\kappa}$ sends exact sequences of shape

$$
\mathcal{A} \longrightarrow \mathcal{B} \longrightarrow \mathcal{B} / \mathcal{A}
$$

to cofiber sequences in $\mathcal{M}_{\text {loc }}^{\kappa}$. Since Idem: $\mathrm{Cat}_{\infty}^{\mathrm{ex}} \rightarrow \mathrm{Cat}_{\infty}^{\text {perf }}$ is a localization, it commutes with colimits and therefore the right-hand vertical map in the diagram

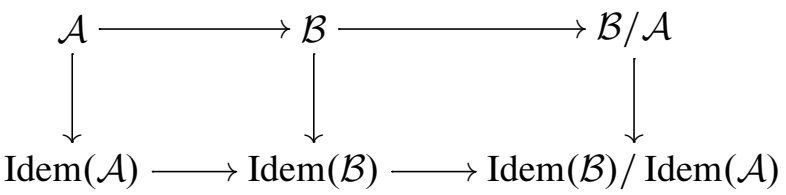

is a Morita equivalence. The bottom line is a strict-exact sequence, and so we conclude that $\mathcal{U}_{\mathrm{loc}}^{\kappa}$ sends exact sequences to cofiber sequences. Finally, the universality of $\mathcal{U}_{\text {loc }}^{\kappa}$ follows from Propositions 8.3 and 8.5 and from the universal property of localization (see Section 2.5).

\subsection{Universal localizing invariant}

We denote by $\mathcal{E}_{\mathrm{L}}$ the small set of maps in $\mathcal{M}_{\text {loc }}^{\kappa}$ of shape

$$
\operatorname{colim}_{\alpha} \mathcal{U}_{\mathrm{loc}}^{\kappa}\left(\mathcal{A}_{\alpha}\right) \longrightarrow \mathcal{U}_{\mathrm{loc}}^{\kappa}(\mathcal{A}),
$$

where $\left\{\mathcal{A}_{\alpha}\right\}$ is a filtered diagram of objects in $\left(\mathrm{Cat}_{\infty}^{\mathrm{ex}}\right)^{\omega}$ whose colimit is a $\kappa$-compact small stable $\infty$-category $\mathcal{A}$. Localize $\mathcal{M}_{\text {loc }}^{\kappa}$ with respect to the set $\mathcal{E}_{\mathrm{L}}$, and let $\mathcal{U}_{\text {loc }}$ be the following composition

$$
\mathrm{Cat}_{\infty}^{\mathrm{ex}} \stackrel{\mathcal{U}_{\mathrm{loc}}^{\kappa}}{\longrightarrow} \mathcal{M}_{\mathrm{loc}}^{\kappa} \stackrel{\gamma}{\longrightarrow} \mathcal{M}_{\mathrm{loc}},
$$

where $\gamma$ is the localization functor.

Theorem 8.7 The functor $\mathcal{U}_{\text {loc }}$ is the universal localizing invariant, ie given any stable presentable $\infty$-category $\mathcal{D}$, we have an equivalence of $\infty$-categories

$$
\left(\mathcal{U}_{\text {loc }}\right)^{*}: \operatorname{Fun}^{\mathrm{L}}\left(\mathcal{M}_{\text {loc }}, \mathcal{D}\right) \stackrel{\sim}{\longrightarrow} \operatorname{Fun}_{\text {loc }}\left(\mathrm{Cat}_{\infty}^{\mathrm{ex}}, \mathcal{D}\right) .
$$


Proof Let us denote by $\mathcal{M}_{\text {add }}^{\kappa}$ the small stable $\infty$-category constructed as in Section 6 but where we use $\left(\mathrm{Cat}_{\infty}^{\text {perf }}\right)^{\kappa}$ instead of $\left(\mathrm{Cat}_{\infty}^{\text {perf }}\right)^{\omega}$. Similarly to $\mathcal{U}_{\text {add }}$ we have a welldefined functor $\mathcal{U}_{\text {add }}^{\kappa}$ : $\mathrm{Cat}_{\infty}^{\mathrm{ex}} \rightarrow \mathcal{M}_{\text {add }}^{\kappa}$ and so by performing a localization analogous to the one of Section 8.3 (with $\mathcal{E}_{A}$ instead of $\mathcal{E}_{\mathrm{L}}$ ) we obtain a small stable $\infty$-category which we denote by $\mathcal{M}_{\text {add }}^{\omega}$ and a composed functor

$$
\mathcal{U}_{\mathrm{add}}^{\omega}: \mathrm{Cat}_{\infty}^{\mathrm{ex}} \stackrel{\mathcal{U}_{\mathrm{add}}^{\kappa}}{\longrightarrow} \mathcal{M}_{\mathrm{add}}^{\kappa} \stackrel{\gamma}{\longrightarrow} \mathcal{M}_{\mathrm{add}}^{\omega} .
$$

Let us start by showing that $\mathcal{M}_{\text {add }}^{\omega}$ agrees with $\mathcal{M}_{\text {add }}$ and that $\mathcal{U}_{\text {add }}^{\omega}$ agrees with $\mathcal{U}_{\text {add }}$. For this (and because of the universal property of $\mathcal{U}_{\text {add }}$ and $\mathcal{U}_{\text {add }}^{\omega}$ ) it suffices to show that $\mathcal{U}_{\text {add }}^{\omega}$ preserves filtered colimits. Consider the composite

$$
\left(\mathrm{Cat}_{\infty}^{\mathrm{perf}}\right)^{\omega} \hookrightarrow\left(\mathrm{Cat}_{\infty}^{\mathrm{perf}}\right)^{\kappa} \subset \mathrm{Cat}_{\infty}^{\mathrm{ex}} \stackrel{\mathcal{U}_{\mathrm{add}}^{\kappa}}{\longrightarrow} \mathcal{M}_{\mathrm{add}}^{\kappa}
$$

It inverts Morita equivalences and sends split-exact sequences to split cofiber sequences. By the construction of $\mathcal{M}_{\text {add }}$ we obtain then an additive invariant $\mathrm{Cat}_{\infty}^{\mathrm{ex}} \rightarrow \mathcal{M}_{\text {add }}^{\kappa}$ and hence by the universal property of $\mathcal{U}_{\text {add }}$ a colimit preserving functor $\Phi: \mathcal{M}_{\text {add }} \rightarrow \mathcal{M}_{\text {add }}^{\kappa}$ and a natural transformation $\eta$ : $\Phi \circ \mathcal{U}_{\text {add }} \Rightarrow \mathcal{U}_{\text {add }}^{\kappa}$. We now observe that the two functors

$$
\mathrm{Cat}_{\infty}^{\mathrm{ex}} \stackrel{\mathcal{U}_{\mathrm{add}}^{\kappa}}{\longrightarrow} \mathcal{M}_{\mathrm{add}}^{\kappa} \stackrel{\gamma}{\longrightarrow} \mathcal{M}_{\mathrm{add}}^{\omega} \quad \text { and } \quad \mathrm{Cat}_{\infty}^{\mathrm{ex}} \stackrel{\mathcal{U}_{\mathrm{add}}}{\longrightarrow} \mathcal{M}_{\mathrm{add}} \stackrel{\Phi}{\longrightarrow} \mathcal{M}_{\mathrm{add}}^{\kappa} \stackrel{\gamma}{\longrightarrow} \mathcal{M}_{\mathrm{add}}^{\omega}
$$

agree. Since they preserve $\kappa$-filtered colimits and every object in $\mathrm{Cat}_{\infty}^{\mathrm{ex}}$ can be expressed as a $\kappa$-filtered colimit of $\kappa$-small objects, it suffices to show that they agree for every $\kappa$-compact small stable $\infty$-category $\mathcal{A}$. The stable $\infty$-category $\mathcal{A}$ can be expressed as a filtered colimit $\operatorname{colim}_{\alpha}\left(\mathcal{A}_{\alpha}\right) \rightarrow \mathcal{A}$, with $\mathcal{A}_{\alpha} \in\left(\mathrm{Cat}_{\infty}^{\mathrm{ex}}\right)^{\omega}$, and the evaluation of the natural transformation $\eta$ at $\mathcal{A}$ identifies with

$$
\operatorname{colim}_{\alpha} \mathcal{U}_{\mathrm{add}}^{\kappa}\left(\mathcal{A}_{\alpha}\right) \longrightarrow \mathcal{U}_{\mathrm{add}}^{\kappa}(\mathcal{A}) .
$$

Since these map belongs to $\mathcal{E}_{A}$, they become invertible in $\mathcal{M}_{\text {add }}^{\omega}$, and so we conclude that the above two functors agree. Since the one on the right-hand side preserves filtered colimits we conclude that $\mathcal{U}_{\text {add }}^{\omega}$ also preserves filtered colimits. This shows that $\mathcal{U}_{\text {add }}^{\omega}$ agrees with $\mathcal{U}_{\text {add }}$ (and hence that $\mathcal{M}_{\text {add }}^{\omega}$ agrees with $\mathcal{M}_{\text {add }}$ ). Now, let $\mathcal{M}_{\text {loc }}^{\omega}$ be the category defined as $\mathcal{M}_{\text {loc }}^{\kappa}$ but with $\kappa$ replaced by $\omega$. Clearly, the associated functor $\mathcal{U}_{\text {loc }}^{\omega}$ is the universal localizing invariant and so in order to conclude the proof of Theorem 8.7 it suffices to show that $\mathcal{M}_{\text {loc }}$ agrees with $\mathcal{M}_{\text {loc }}^{\omega}$ and that $\mathcal{U}_{\text {loc }}$ agrees with $\mathcal{U}_{\mathrm{loc}}^{\omega}$. Starting with $\mathcal{M}_{\text {add }}^{\kappa}$ we can perform the following two localizations:

$$
\mathcal{M}_{\mathrm{add}}^{\kappa} \longrightarrow \mathcal{M}_{\mathrm{loc}}^{\kappa} \longrightarrow \mathcal{M}_{\mathrm{loc}}^{\omega} \text { and } \mathcal{M}_{\mathrm{add}}^{\kappa} \longrightarrow \mathcal{M}_{\mathrm{add}}^{\omega} \simeq \mathcal{M}_{\mathrm{add}} \longrightarrow \mathcal{M}_{\mathrm{loc}}
$$

Since these localizations are independent of the order in which they are performed, our claim follows and so the proof is finished. 


\section{Nonconnective $K$-theory}

Bass introduced the negative $K$-groups in order to measure the failure of $K_{0}$ and $K_{1}$ to satisfy localization; this perspective was studied in detail in Thomason-Trobaugh and led to the definition of the Bass-Thomason nonconnective $K$-theory spectrum of rings and schemes. In fact, any nontrivial theory which is "like $K$-theory" and satisfies localization must be nonconnective; there is a nice discussion of this in [44]. In this section we introduce the nonconnective algebraic $K$-theory of $\infty$-categories and show that it becomes corepresentable in $\mathcal{M}_{\text {loc }}$; see Theorem 9.8. This result depends critically on the multistage construction of Section 8 , which again follows the general pattern of the argument for $\mathrm{dg}$-categories in [21]. For a connective ring spectrum $R$, we give a slightly different definition of the nonconnective $K$-theory in terms of a "suspension ring spectrum" of $R$, and use this show that the negative $K$-groups of $R$ are isomorphic to those of $\pi_{0} R$.

\subsection{Nonconnective $K$-theory of $\infty$-categories}

In order to construct the nonconnective $K$-theory spectrum associated to a small stable $\infty$-category, we use a generalization of the axiomatic framework due to Schlichting [64]. For an uncountable regular cardinal $\kappa$, we will produce functors $\mathcal{F}_{\kappa}$ and $\Sigma_{\kappa}$ from $\mathrm{Cat}_{\infty}^{\mathrm{ex}}$ to $\mathrm{Cat}_{\infty}^{\mathrm{ex}}$ such that for any small stable $\infty$ category $\mathcal{A}$ :

(i) $K\left(\mathcal{F}_{\kappa} \mathcal{A}\right)$ is contractible.

(ii) There are natural transformations

$$
\mathrm{Id} \longrightarrow \mathcal{F}_{\kappa} \longrightarrow \Sigma_{\kappa}
$$

such that $\mathcal{A} \rightarrow \mathcal{F}_{\kappa} \mathcal{A} \rightarrow \Sigma_{\kappa} \mathcal{A}$ is exact.

(iii) The functors $\mathcal{F}_{\kappa}$ and $\Sigma_{\kappa}$ preserve exact sequences.

(iv) $\mathcal{F}_{\kappa}$ and $\Sigma_{\kappa}$ preserve $\kappa$-filtered colimits in $\mathrm{Cat}_{\infty}^{\mathrm{ex}}$.

The idea is that $\mathcal{F}_{\kappa} \mathcal{A}$ is a " $K$-theoretic cone" and so $\Sigma_{\kappa} \mathcal{A}$ is a "suspension" of $\mathcal{A}$. Fix an uncountable regular cardinal $\kappa$, and for a stable $\infty$-category $\mathcal{C}$ recall from Section 2.4 that $\mathcal{C}^{\kappa}$ denotes the $\kappa$-compact objects in $\mathcal{C}$.

Definition 9.1 Using Proposition 2.18, we define $\mathcal{F}_{\kappa} \mathcal{A}=\left(\operatorname{Ind}_{\omega}(\mathcal{A})\right)^{\kappa}$ and $\Sigma_{\kappa} \mathcal{A}$ to be the cofiber $\left(\operatorname{Ind}_{\omega}(\mathcal{A})\right)^{\kappa} / \mathcal{A}$.

Remark 9.2 One might wish to simply use $\operatorname{Ind}_{\omega} \mathcal{A}$ as the cone construction; however, this will rarely turn out to be a small $\infty$-category, whereas passing to the $\kappa$-compact objects yields an (essentially) small $\infty$-category by construction. 
Observe that $\mathcal{F}_{\kappa}$ is a composite functor

$$
\mathrm{Cat}_{\infty}^{\mathrm{ex}} \longrightarrow \mathcal{P r}_{\mathrm{St} \omega}^{\mathrm{L}} \longrightarrow \mathrm{Cat}_{\infty}^{\mathrm{ex}(\kappa)} \longrightarrow \mathrm{Cat}_{\infty}^{\mathrm{ex}}
$$

By construction and Propositions 5.6 and 5.9, we have an exact sequence

$$
\mathcal{A} \longrightarrow \mathcal{F}_{\kappa} \mathcal{A} \longrightarrow \Sigma_{\kappa} \mathcal{A},
$$

which is natural in small stable $\infty$-categories $\mathcal{A}$. Next, we check that $\mathcal{F}_{\kappa} \mathcal{A}$ satisfies property (i) above.

Lemma 9.4 Let $\mathcal{A}$ be a small stable $\infty$-category. Then $K\left(\mathcal{F}_{\kappa} \mathcal{A}\right)$ is trivial.

Proof Since $\kappa$ is uncountable, $\mathcal{F}_{\kappa} \mathcal{A}$ has countable coproducts, and so the usual Eilenberg swindle argument implies that the identity map is null-homotopic on $K_{-}$ theory and so its $K$-theory vanishes. Specifically, the functor $F: \mathcal{F}_{K} \mathcal{A} \rightarrow \mathcal{F}_{K} \mathcal{A}$ defined by $X \mapsto \coprod_{\mathbb{N}} X$ is exact. Moreover, there is a natural equivalence of exact functors id $\sqcup F \simeq F$ induced by the equivalence $X \sqcup\left(\coprod_{\mathbb{N}} X\right) \simeq \coprod_{\mathbb{N}} X$. Applying $K$-theory, we can split off the $F$ component of the resulting equivalence of spectra and deduce that the identity of $\mathcal{F}_{K} \mathcal{A}$ is null-homotopic.

We must check that $\mathcal{F}_{\kappa}$ and $\Sigma_{\kappa}$ preserve exact sequences of small stable $\infty$-categories.

Proposition 9.5 Let $\mathcal{A} \rightarrow \mathcal{B} \rightarrow \mathcal{C}$ be an exact sequence of small stable $\infty$-categories. Then the induced sequences

$$
\mathcal{F}_{\kappa} \mathcal{A} \longrightarrow \mathcal{F}_{\kappa} \mathcal{B} \longrightarrow \mathcal{F}_{\kappa} \mathcal{C} \quad \text { and } \quad \Sigma_{\kappa} \mathcal{A} \longrightarrow \Sigma_{\kappa} \mathcal{B} \longrightarrow \Sigma_{\kappa} \mathcal{C}
$$

are exact.

Proof It suffices to show the result for $\mathcal{F}_{\kappa}$, as the statement for $\Sigma_{\kappa}$ follows because colimits commute. Thus, we need to verify that

$$
\left(\operatorname{Ind}_{\omega}(\mathcal{A})\right)^{\kappa} \longrightarrow\left(\operatorname{Ind}_{\omega}(\mathcal{B})\right)^{\kappa} \longrightarrow\left(\operatorname{Ind}_{\omega}(\mathcal{C})\right)^{\kappa}
$$

is exact. Definition 5.12 and Proposition 5.15 show that the sequence

$$
\operatorname{Ind}_{\omega} \mathcal{A} \longrightarrow \operatorname{Ind}_{\omega} \mathcal{B} \longrightarrow \operatorname{Ind}_{\omega} \mathcal{C}
$$

is exact. Now the result follows from Proposition 5.17.

Passing to the triangulated homotopy category by composing with the functor Ho, we get a series of functors which satisfies Schlichting's setup of [64, Section 2.2] and so produces negative $K$-groups. Furthermore, we can define the nonconnective $K$-theory spectrum as follows, following [64, Section 12]. 
Definition 9.6 Let $\mathcal{A}$ be a small stable $\infty$-category. Its nonconnective $K$-theory spectrum $K(\mathcal{A})$ is given by

$$
\boldsymbol{K}(\mathcal{A}):=\operatorname{colim}_{n} \Omega^{n} K\left(\Sigma_{\kappa}^{(n)}(\mathcal{A})\right) .
$$

Here $K$ stands for the $K$-theory spectrum of Section 7.1, and the structure maps are induced from the exact sequences

$$
\Sigma_{\kappa}^{(n)}(\mathcal{A}) \longrightarrow \mathcal{F}_{\kappa} \Sigma_{\kappa}^{(n)}(\mathcal{A}) \longrightarrow \Sigma_{\kappa}^{(n+1)}(\mathcal{A}), \quad n \geq 0 .
$$

Schlichting's axiomatic framework implies that this construction agrees with his when both are defined, and therefore we deduce from his comparison results [64, Section 8] that the nonconnective $K$-theory spectrum of Definition 9.6 agrees with the various classical constructions of nonconnective $K$-theory spectra.

Finally, we establish the final technical condition; this will be needed in the following sections.

Lemma 9.7 The functors $\mathcal{F}_{\kappa}$ and $\Sigma_{\kappa}$ preserve $\kappa$-filtered colimits.

Proof Recall that $\mathcal{F}_{\kappa}$ is the composite (9.3). Hence, the claim follows from the fact that the passage to $\operatorname{Ind}_{\kappa}$ and to $\kappa$-compact objects preserves $\kappa$-filtered colimits [47, 5.5.7.8, 5.5.7.10, 5.5.7.11]. Since $\Sigma_{\kappa}$ is the cofiber of the inclusion $\mathcal{A} \rightarrow \mathcal{F}_{\kappa}$ and colimits commute, we deduce that $\Sigma_{\kappa}$ preserves $\kappa$-filtered colimits if $\mathcal{F}_{\kappa}$ does.

\subsection{Corepresentability}

This subsection is entirely devoted to the proof of the following corepresentability result.

Theorem 9.8 Let $\mathcal{A}$ be a small stable $\infty$-category. Then there is a natural equivalence of spectra

$$
\operatorname{Map}\left(\mathcal{U}_{\text {loc }}\left(\mathcal{S}_{\infty}^{\omega}\right), \mathcal{U}_{\text {loc }}(\mathcal{A})\right) \simeq \boldsymbol{K}(\mathcal{A})
$$

In particular, for each integer $n$, we have isomorphisms of abelian groups

$$
\operatorname{Hom}\left(\mathcal{U}_{\text {loc }}\left(\mathcal{S}_{\infty}^{\omega}\right), \Sigma^{-n} \mathcal{U}_{\text {loc }}(\mathcal{A})\right) \simeq \boldsymbol{K}_{n}(\mathcal{A})
$$

in the triangulated category $\operatorname{Ho}\left(\mathcal{M}_{\mathrm{loc}}\right)$.

The proof of Theorem 9.8 will follow from Theorems 9.9 and 9.10 and from Propositions 9.17 through 9.26 . 
Theorem 9.9 Let $\mathcal{A}$ and $\mathcal{B}$ be small stable $\infty$-categories such that $\mathcal{B}$ is $\kappa$-compact. Then there is a natural equivalence of spectra

$$
\operatorname{Map}\left(\underline{\mathcal{U}}_{\text {add }}^{\kappa}(\mathcal{B}), \underline{\mathcal{U}}_{\text {add }}^{\kappa}(\mathcal{A})\right) \simeq K\left(\operatorname{Fun}{ }^{\mathrm{ex}}(\mathcal{B}, \mathcal{A})\right) .
$$

If $\mathcal{B}=\mathcal{S}_{\infty}^{\omega}$ is the $\infty$-category of compact spectra, this reduces to an equivalence

$$
\operatorname{Map}\left(\underline{\mathcal{U}}_{\text {add }}^{\kappa}\left(\mathcal{S}_{\infty}^{\omega}\right), \underline{\mathcal{U}}_{\text {add }}^{\kappa}(\mathcal{A})\right) \simeq K(\mathcal{A}) .
$$

Proof The proof is analogous to the argument for Theorem 7.13; instead of the idempotent-complete stable $\infty$-category $\operatorname{Fun}^{\operatorname{ex}}(\mathcal{B}, \operatorname{Idem}(\mathcal{A}))$ we consider the small stable $\infty$-category $\operatorname{Fun}^{\mathrm{ex}}(\mathcal{B}, \mathcal{A})$. Note that since $\kappa>\omega, \mathcal{S}_{\infty}^{\omega}$ belongs to $\left(\mathrm{Cat}_{\infty}^{\mathrm{ex}}\right)^{\kappa}$.

Theorem 9.10 Let $\mathcal{A}$ be a small stable $\infty$-category. Then there is a natural equivalence of spectra

$$
\operatorname{Map}\left(\underline{\mathcal{U}}_{\text {wloc }}^{\kappa}\left(\mathcal{S}_{\infty}^{\omega}\right), \underline{\mathcal{U}}_{\text {wloc }}^{\kappa}(\mathcal{A})\right) \simeq K(\mathcal{A}) .
$$

Proof By construction, the object $\underline{\mathcal{U}}_{\text {add }}^{\kappa}\left(\mathcal{S}_{\infty}^{\omega}\right)$ is compact in $\underline{\mathcal{M}}_{\text {add }}^{\kappa}$. Let $S$ denote the set of maps in (8.4), $\bar{S}$ the strongly saturated collection of arrows generated by $S$ [47, 5.5.4.5], and let $X$ be an $S$-local object such that the map $\underline{\mathcal{U}}_{\text {add }}^{\kappa}(\mathcal{A}) \rightarrow X$ is an $S$-local equivalence (ie $\underline{\mathcal{U}}_{\text {add }}^{\kappa}(\mathcal{A}) \rightarrow X$ is in $\bar{S}$ ). Then by definition,

$$
\operatorname{Map}\left(\underline{\mathcal{U}}_{\text {wloc }}^{\kappa}\left(\mathcal{S}_{\infty}^{\omega}\right), \underline{\mathcal{U}}_{\text {wloc }}^{\kappa}(\mathcal{A})\right) \simeq \operatorname{Map}\left(\underline{\mathcal{U}}_{\text {add }}^{\kappa}\left(\mathcal{S}_{\infty}^{\omega}\right), X\right),
$$

so it suffices to show that the functor

$$
R:=\operatorname{Map}\left(\underline{\mathcal{U}}_{\text {add }}^{\kappa}\left(\mathcal{S}_{\infty}^{\omega}\right),-\right): \underline{\mathcal{M}}_{\text {add }}^{\kappa} \longrightarrow \mathcal{S}_{\infty}
$$

sends the maps in $\bar{S}$ to equivalences of spectra. Since $\underline{\mathcal{M}}_{\text {add }}^{\kappa}$ is a stable $\infty$-category and $\underline{\mathcal{U}}_{\text {add }}^{\kappa}\left(\mathcal{S}_{\infty}^{\omega}\right)$ is compact, $R$ preserves small colimits, so the two-out-of-three property allows us to reduce to checking that $R$ sends the elements of $S$ to equivalences.

Consider the following diagram:

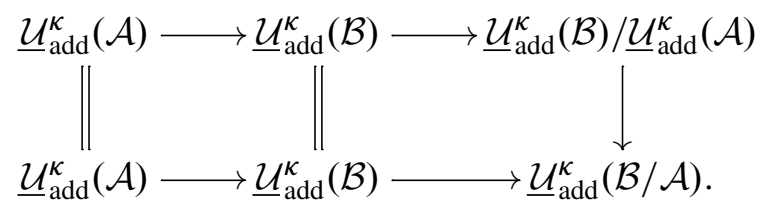

By applying the functor (9.11) to the above diagram (9.12) we obtain by Theorem 9.9 a diagram in $\mathcal{S}$,

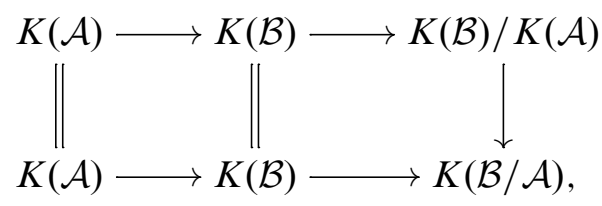


where the upper row is a homotopy cofiber sequence. Now, an argument analogous to the one used in the proof of Proposition 7.19 (where we make use of Waldhausen's Fibration Theorem) allow us to conclude that the lower row in the above diagram (9.13) is also a homotopy cofiber sequence. This completes the argument.

Let $\mathbf{V}$ be the partially ordered set $\{(i, j):|i-j| \leq 1, i, j \geq 0\} \subset \mathbb{N} \times \mathbb{N}$. Given a small stable $\infty$-category $\mathcal{A}$, we denote by $\operatorname{Dia}(\mathcal{A})$ the $\mathrm{N}(\mathbf{V})$-diagram

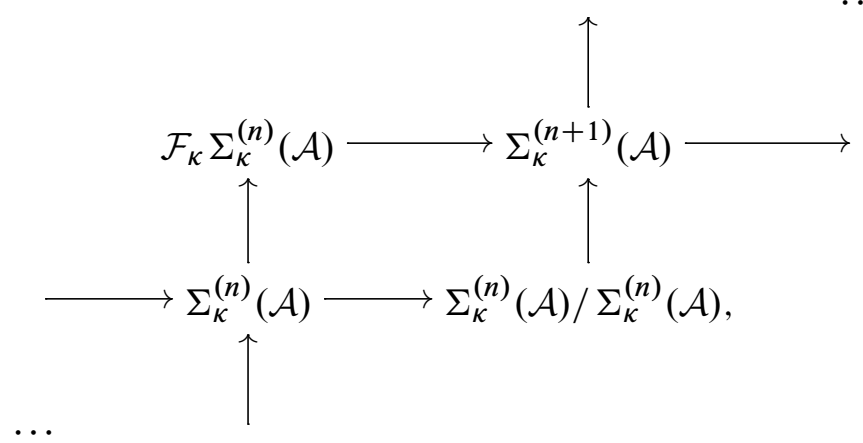

where $\mathcal{F}_{\kappa}$ and $\Sigma_{\kappa}$ are as in Definition 9.1.

Lemma 9.15 Let $\mathcal{A}$ be a small stable $\infty$-category. Then $\Sigma_{\kappa}^{(n)}(\mathcal{A}) / \Sigma_{\kappa}^{(n)}(\mathcal{A})$ and $\mathcal{F}_{\kappa} \Sigma_{\kappa}^{(n)}(\mathcal{A})$ become trivial after application of $\underline{\mathcal{U}}_{\text {wloc }}^{\kappa}$.

Proof The object $\Sigma_{\kappa}^{(n)}(\mathcal{A}) / \Sigma_{\kappa}^{(n)}(\mathcal{A})$ is already trivial in $\mathrm{Cat}_{\infty}^{\mathrm{ex}}$. Since Proposition 2.18 implies that $\mathcal{F}_{\kappa} \Sigma_{\kappa}^{(n)}(\mathcal{A})$ admits all $\kappa$-small colimits, for any $\mathcal{B}$ in $\left(\mathrm{Cat}_{\infty}^{\mathrm{ex}}\right)^{\kappa}$ the small stable $\infty$-category $\operatorname{Fun}\left(\mathcal{B}, \mathcal{F}_{\kappa} \Sigma_{\kappa}^{(n)}(\mathcal{A})\right)$ also admits all $\kappa$-small colimits. Thus, the connective $K$-theory spectrum $K\left(\operatorname{Fun}\left(\mathcal{B}, \mathcal{F}_{\kappa} \Sigma_{\kappa}^{(n)}(\mathcal{A})\right)\right.$ is trivial. Finally, Theorem 9.9 and the fact that the objects $\underline{\mathcal{U}}_{\text {add }}^{\kappa}(\mathcal{B})$, with $\mathcal{B}$ in $\left(\mathrm{Cat}_{\infty}^{\mathrm{ex}}\right)^{\kappa}$ generate the category $\underline{\mathcal{M}}_{\text {add }}^{\kappa}$ $[47,5.5 .7 .3]$ allow us to conclude that $\mathcal{F}_{\kappa} \Sigma_{\kappa}^{(n)}(\mathcal{A})$ becomes trivial after application of $\underline{\mathcal{U}}_{\text {add }}^{\kappa}$, and thus after application of $\underline{\mathcal{U}}_{\text {wloc }}^{\kappa}$.

Let $\mathcal{A}$ be a small stable $\infty$-category. We denote by $V(\mathcal{A})$ the object

$$
V(\mathcal{A})=\operatorname{colim}_{n} \Sigma^{-n} \underline{\mathcal{U}}_{\mathrm{wloc}}^{\kappa}\left(\Sigma_{\kappa}^{(n)}(\mathcal{A})\right)
$$

in $\mathcal{M}_{\text {wloc }}^{\kappa}$ whose indexing maps are induced from the above diagram (9.14). Note that $V(\mathcal{A})$ is functorial in $\mathcal{A}$ and that we have a natural map $\underline{\mathcal{U}}_{\text {wloc }}^{\kappa}(\mathcal{A}) \rightarrow V(\mathcal{A})$. We obtain then a well-defined functor $V$ along with a natural transformation:

$$
V(-): \mathrm{Cat}_{\infty}^{\mathrm{ex}} \longrightarrow \underline{\mathcal{M}}_{\mathrm{wloc}}^{\kappa}, \quad \underline{\mathcal{U}}_{\mathrm{wloc}}^{\kappa} \Rightarrow V(-) .
$$


Proposition 9.17 Let $\mathcal{A}$ be a small stable $\infty$-category. Then, there is a natural equivalence of spectra

$$
\operatorname{Map}\left(\underline{\mathcal{U}}_{\text {wloc }}^{\kappa}\left(\mathcal{S}_{\infty}^{\omega}\right), V(\mathcal{A})\right) \simeq \boldsymbol{K}(\mathcal{A}) .
$$

Proof This follows from the following equivalences:

$$
\begin{aligned}
\boldsymbol{K}(\mathcal{A}) & =\underset{n \geq 0}{\operatorname{hocolim}} \Omega^{n} K\left(\Sigma_{\kappa}^{(n)}(\mathcal{A})\right) \\
& \simeq \underset{n \geq 0}{\operatorname{hocolim}} \Omega^{n} \operatorname{Map}\left(\underline{\mathcal{U}}_{\mathrm{wloc}}^{\kappa}\left(\mathcal{S}_{\infty}^{\omega}\right), \underline{\mathcal{U}}_{\mathrm{wloc}}^{\kappa}\left(\Sigma_{\kappa}^{(n)}(\mathcal{A})\right)\right) \\
& \simeq \operatorname{Map}\left(\underline{\mathcal{U}}_{\mathrm{wloc}}^{\kappa}\left(\mathcal{S}_{\infty}\right)^{\kappa}, \underset{n \geq 0}{\operatorname{colim}} \Sigma^{-n} \underline{\mathcal{U}}_{\mathrm{wloc}}^{\kappa}\left(\Sigma_{\kappa}^{(n)}(\mathcal{A})\right)\right) \\
& \simeq \operatorname{Map}\left(\underline{\mathcal{U}}_{\mathrm{wloc}}^{\kappa}\left(\mathcal{S}_{\infty}^{\kappa}\right), V(\mathcal{A})\right) .
\end{aligned}
$$

Equivalence (9.18) comes from Theorem 9.10 and equivalence (9.19) comes from the compactness of $\underline{\mathcal{U}}_{\text {wloc }}^{\kappa}\left(\mathcal{S}_{\infty}^{\omega}\right)$ in $\underline{\mathcal{M}}_{\text {wloc }}^{\kappa}$.

Proposition 9.20 The functor $V$ (9.16) inverts Morita equivalences.

Proof It suffices to show that $V(-)$ sends maps of shape $\mathcal{A} \rightarrow \operatorname{Idem}(\mathcal{A})$ to isomorphisms. Consider the following diagram:

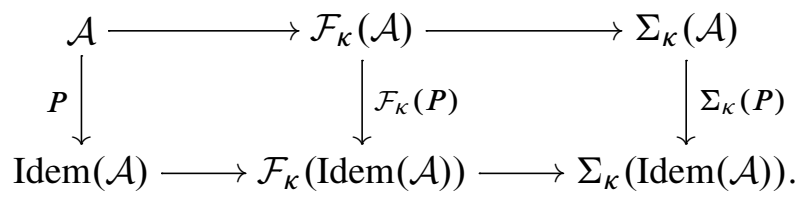

Proposition 2.18 implies that $\mathcal{F}_{\kappa}(P)$ is an equivalence. Therefore, since both rows are strict-exact sequences and $\operatorname{Ho}(\mathcal{A})$ and $\operatorname{Ho}(\operatorname{Idem}(\mathcal{A}))$ differ by direct summands, we conclude that $\Sigma_{\kappa}(P)$ is an equivalence. The definition of the functor $V(-)$ allow us to conclude the proof.

Proposition 9.21 The functor

$$
V(-): \mathrm{Cat}_{\infty}^{\mathrm{ex}} \longrightarrow \underline{\mathcal{M}}_{\text {wloc }}^{\kappa}
$$

inverts Morita equivalences, preserves $\kappa$-filtered colimits and sends exact sequences to cofiber sequences.

Proof Proposition 9.20 implies that $V(-)$ inverts Morita equivalences. Furthermore, by Lemma 9.7, $\mathcal{F}_{\kappa}$ and $\Sigma_{\kappa}$ preserve $\kappa$-filtered colimits for $\kappa>\omega$, and so $V(-)$ does as well. Now, let

$$
\mathcal{A} \longrightarrow \mathcal{B} \longrightarrow \mathcal{C}
$$


be an exact sequence. Proposition 9.20 implies that we can assume that $\operatorname{Ho}(\mathcal{A})$ is a thick triangulated subcategory of $\operatorname{Ho}(\mathcal{B})$. Consider the following diagram:

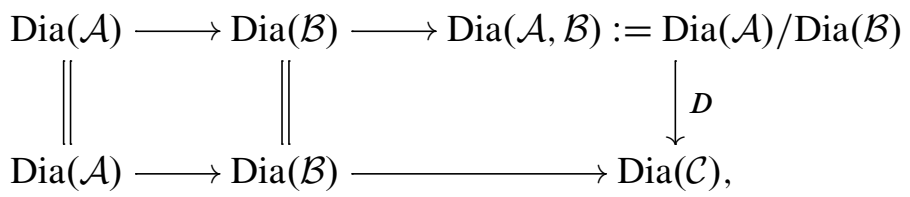

where $\operatorname{Dia}(\mathcal{A}, \mathcal{B})$ is obtained by passage to the cofiber objectwise. Note that since in the above diagram (9.22) the upper row is objectwise a strict-exact sequence, we obtain a cofiber sequence

$$
V(\mathcal{A}) \longrightarrow V(\mathcal{B}) \longrightarrow V(\mathcal{B}, \mathcal{A}) \longrightarrow \Sigma V(\mathcal{A})
$$

in $\underline{\mathcal{M}}_{\text {wloc }}^{\kappa}$, where

$$
V(\mathcal{B}, \mathcal{A}):=\operatorname{colim}_{n} \Sigma^{-n} \underline{\mathcal{U}}_{\mathrm{wloc}}^{\kappa}\left(\Sigma_{\kappa}^{(n)}(\mathcal{B}) / \Sigma_{\kappa}^{(n)}(\mathcal{A})\right) .
$$

We now show that the induced map

$$
V(\mathcal{B}, \mathcal{A}) \longrightarrow V(\mathcal{C})
$$

is an equivalence. For this, consider the following commutative diagram:

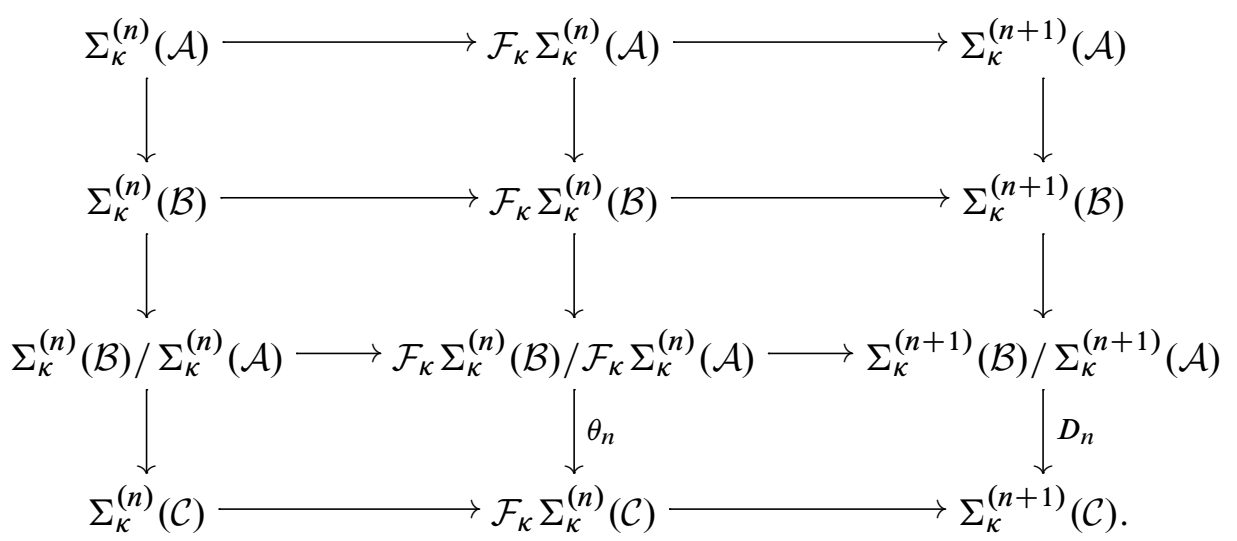

Since the induced triangulated functor

$$
\operatorname{Ho}\left(\mathcal{F}_{\kappa} \Sigma_{\kappa}^{(n)}(\mathcal{A})\right) \longrightarrow \operatorname{Ho}\left(\mathcal{F}_{\kappa} \Sigma_{\kappa}^{(n)}(\mathcal{B})\right)
$$

preserves $\kappa$-small colimits, [64, Section 3.1] implies that the triangulated category

$$
\operatorname{Ho}\left(\mathcal{F}_{\kappa} \Sigma_{\kappa}^{(n)}(\mathcal{B}) / \mathcal{F}_{\kappa} \Sigma_{\kappa}^{(n)}(\mathcal{A})\right)
$$


is idempotent complete. Therefore, $\theta_{n}$ is an equivalence, and we obtain maps

$$
\psi_{n}: \Sigma_{\kappa}^{(n)}(\mathcal{C}) \longrightarrow \mathcal{F}_{\kappa} \Sigma_{\kappa}^{(n)}(\mathcal{B}) / \mathcal{F}_{\kappa} \Sigma_{\kappa}^{(n)}(\mathcal{A})
$$

which induce maps

$$
\Psi_{n}: \Sigma^{-n} \underline{\mathcal{U}}_{\text {wloc }}^{\kappa}\left(\Sigma_{\kappa}^{(n)}(\mathcal{C})\right) \longrightarrow \Sigma^{-n-1} \underline{\mathcal{U}}_{\text {wloc }}^{\kappa}\left(\Sigma_{\kappa}^{(n+1)}(\mathcal{B}) / \Sigma_{\kappa}^{(n+1)}(\mathcal{A})\right) .
$$

It follows that the natural map

$$
\operatorname{colim}_{n} \Sigma^{-n} \underline{\mathcal{U}}_{\text {wloc }}^{\kappa}\left(\Sigma_{\kappa}^{(n)}(\mathcal{B}) / \Sigma_{\kappa}^{(n)}(\mathcal{A})\right) \longrightarrow \operatorname{colim}_{n} \Sigma^{-n} \underline{\mathcal{U}}_{\text {wloc }}^{\kappa}\left(\Sigma_{\kappa}^{(n)}(\mathcal{C})\right)
$$

is an equivalence, which implies that the map (9.23) is an equivalence.

Corollary 9.24 There is a functor

$$
\text { Loc: } \mathcal{M}_{\mathrm{loc}}^{\kappa} \longrightarrow \underline{\mathcal{M}}_{\mathrm{wloc}}^{\kappa}
$$

such that $\operatorname{Loc}\left(\mathcal{U}_{\text {loc }}^{\kappa}(\mathcal{A})\right) \simeq V(\mathcal{A})$, for every small stable $\infty$-category $\mathcal{A}$.

Proof This follows from Propositions 9.21 and 8.6.

Proposition 9.25 The two functors

$$
\text { Loc, } \gamma^{*}: \mathcal{M}_{\text {loc }}^{\kappa} \longrightarrow \underline{\mathcal{M}}_{\text {wloc }}^{\kappa}
$$

are canonically equivalent, where $\gamma^{*}$ is the right adjoint of the localization functor.

Proof Let us denote by $\boldsymbol{L}$ the endofunctor Loc $\circ \gamma$ of $\underline{\mathcal{M}}_{\text {wloc }}^{\kappa}$. Note that we have a natural transformation Id $\Rightarrow \boldsymbol{L}$. Making use of the definition of $V(-)$ and of the fact that colimits in $\infty$-categories commute, we observe that $\boldsymbol{L}$ is a localization functor on $\underline{\mathcal{M}}_{\text {wloc }}^{\kappa}[47,5.2 .7 .4]$. Therefore, it suffices to show that a map in $\underline{\mathcal{M}}_{\text {wloc }}^{\kappa}$ becomes an equivalence in $\mathcal{M}_{\text {loc }}^{\kappa}$ if and only if it becomes an equivalence after application of $\boldsymbol{L}$. This follows from the fact that for every small stable $\infty$-category $\mathcal{A}$, we have an equivalence $\gamma(V(\mathcal{A})) \simeq \mathcal{U}_{\text {loc }}^{\kappa}(\mathcal{A})$ : note that we have cofiber sequences in $\mathcal{M}_{\text {loc }}^{\kappa}$

$$
\mathcal{U}_{\mathrm{loc}}^{\kappa}\left(\Sigma_{\kappa}^{(n)}(\mathcal{A})\right) \longrightarrow \mathcal{U}_{\mathrm{loc}}^{\kappa}\left(\mathcal{F}_{\kappa} \Sigma_{\kappa}^{(n)}(\mathcal{A})\right) \longrightarrow \mathcal{U}_{\mathrm{loc}}^{\kappa}\left(\Sigma_{\kappa}^{(n+1)}(\mathcal{A})\right) .
$$

Proposition 9.26 Let $\mathcal{A}$ be small stable $\infty$-category. We have a natural isomorphism in the stable homotopy category of spectra,

$$
\operatorname{Map}\left(\mathcal{U}_{\mathrm{loc}}^{\kappa}\left(\mathcal{S}_{\infty}^{\omega}\right), \mathcal{U}_{\mathrm{loc}}^{\kappa}(\mathcal{A})\right) \simeq \boldsymbol{K}(\mathcal{A})
$$


Proof This follows from the following equivalences:

$$
\begin{aligned}
\operatorname{Map}\left(\mathcal{U}_{\mathrm{loc}}^{\kappa}\left(\mathcal{S}_{\infty}^{\omega}\right), \mathcal{U}_{\mathrm{loc}}^{\kappa}(\mathcal{A})\right) & \simeq \operatorname{Map}\left(\mathcal{U}_{\mathrm{wloc}}^{\kappa}\left(\mathcal{S}_{\infty}^{\omega}\right), \gamma^{*}\left(\mathcal{U}_{\mathrm{loc}}^{\kappa}(\mathcal{A})\right)\right) \\
& \simeq \operatorname{Map}\left(\underline{\mathcal{U}}_{\mathrm{wloc}}^{\kappa}\left(\mathcal{S}_{\infty}^{\omega}\right), \operatorname{Loc}\left(\mathcal{U}_{\mathrm{loc}}^{\kappa}(\mathcal{A})\right)\right) \\
& \simeq \operatorname{Map}\left(\underline{\mathcal{U}}_{\mathrm{wloc}}^{\kappa}\left(\mathcal{S}_{\infty}^{\omega}\right), V(\mathcal{A})\right) \\
& \simeq \boldsymbol{K}(\mathcal{A}) .
\end{aligned}
$$

Equivalence (9.27) comes from Proposition 9.25, equivalence (9.28) comes from Corollary 9.24, and equivalence (9.29) is Proposition 9.17.

Proof of Theorem 9.8 Recall from Section 8.3 that $\mathcal{M}_{\text {loc }}$ is obtained by localizing $\mathcal{M}_{\text {loc }}^{\kappa}$ with respect to the set $\mathcal{E}_{\mathrm{L}}$. Since $\mathcal{U}_{\text {loc }}^{\kappa}\left(\mathcal{S}_{\infty}^{\omega}\right)$ is compact in $\mathcal{M}_{\text {loc }}^{\kappa}$, it is sufficient by Proposition 9.26 and the universal property of localization (see Section 2.5) to show that the functor

$$
\operatorname{Map}\left(\mathcal{U}_{\mathrm{loc}}^{\kappa}\left(\mathcal{S}_{\infty}^{\omega}\right),-\right): \mathcal{M}_{\mathrm{loc}}^{\kappa} \longrightarrow \mathcal{S}_{\infty}
$$

sends the elements of $\mathcal{E}_{\mathrm{L}}$ to equivalences. This follows from the fact that the nonconnective $K$-theory construction preserves filtered colimits (see [64, Section 7, Lemma 6]), and so the proof is finished.

\subsection{Nonconnective $K$-theory of Waldhausen categories and localization}

In particular, Theorem 9.8 implies that nonconnective $K$-theory satisfies localization. This is an extremely useful fact in practice; localization sequences provide one of the main computation tools for understanding algebraic $K$-theory. As such, we state a version of this result in terms of Waldhausen categories. We begin by defining the nonconnective $K$-theory of a Waldhausen category.

Definition 9.30 Let $\mathcal{C}$ be a DHKS-saturated Waldhausen category with factorization. Then the nonconnective $K$-theory $\boldsymbol{K}(\mathcal{C})$ of $\mathcal{C}$ is defined as the nonconnective $K$-theory $\boldsymbol{K}\left(\mathrm{N}(\mathcal{C})\left[W^{-1}\right]\left[\Sigma^{-1}\right]\right)$ of the $\infty$-category

$$
\mathrm{N}(\mathcal{C})\left[W^{-1}\right]\left[\Sigma^{-1}\right] \simeq \operatorname{colim}\left\{\mathrm{N}(\mathcal{C})\left[W^{-1}\right] \stackrel{\Sigma}{\longrightarrow} \mathrm{N}(\mathcal{C})\left[W^{-1}\right] \stackrel{\Sigma}{\longrightarrow} \cdots\right\}
$$

obtained by inverting the suspension on the underlying $\infty$-category $\mathrm{N}(\mathcal{C})\left[W^{-1}\right]$ in the $\infty$-category $\mathrm{Cat}_{\infty}^{\mathrm{Rex}}$ of $\infty$-categories with finite colimits and right-exact functors.

This definition in terms of the stabilization is reasonable because of the following consistency results. 
Proposition 9.31 Let $\mathcal{C}$ be a presentable $\infty$-category with a zero object, and let $\mathcal{C}^{\omega}\left[\Sigma^{-1}\right]$ denote the colimit

$$
\mathcal{C}^{\omega}\left[\Sigma^{-1}\right] \simeq \operatorname{colim}\left\{\mathcal{C}^{\omega} \stackrel{\Sigma}{\longrightarrow} \mathcal{C}^{\omega} \stackrel{\Sigma}{\longrightarrow} \cdots\right\}
$$

in $\mathrm{Cat}_{\infty}^{\mathrm{Rex}}$. Then $\mathcal{C}^{\omega}\left[\Sigma^{-1}\right]$ is stable, and the induced functor

$$
\mathcal{C}^{\omega}\left[\Sigma^{-1}\right] \longrightarrow \operatorname{Stab}(\mathcal{C})
$$

identifies the idempotent-completion of $\mathcal{C}^{\omega}\left[\Sigma^{-1}\right]$ with $\operatorname{Stab}(\mathcal{C})^{\omega}$.

Proof Let $\mathcal{D}$ be an idempotent-complete stable $\infty$-category. Then

$$
\begin{aligned}
\operatorname{Fun}^{\mathrm{ex}}\left(\mathcal{C}^{\omega}\left[\Sigma^{-1}\right], \mathcal{D}\right) & \simeq \lim \operatorname{Fun}^{\mathrm{ex}}\left(\mathcal{C}^{\omega}, \mathcal{D}\right) \\
& \simeq \lim \operatorname{Fun}_{\omega}^{\mathrm{L}}(\mathcal{C}, \operatorname{Ind}(\mathcal{D})) \\
& \simeq \operatorname{Fun}_{\omega}^{\mathrm{L}}(\operatorname{Stab}(\mathcal{C}), \operatorname{Ind}(\mathcal{D})) \simeq \operatorname{Fun}^{\mathrm{ex}}\left(\operatorname{Stab}(\mathcal{C})^{\omega}, \mathcal{D}\right) .
\end{aligned}
$$

Since $\operatorname{Stab}(\mathcal{C})^{\omega}$ is necessarily idempotent-complete, we conclude that it is equivalent to the idempotent-completion of $\mathcal{C}^{\omega}\left[\Sigma^{-1}\right]$.

Proposition 9.32 Let $\mathcal{C}$ be a DHKS-saturated Waldhausen category with factorization. Then the natural map $\mathrm{N}(\mathcal{C})\left[W^{-1}\right] \rightarrow \mathrm{N}(\mathcal{C})\left[W^{-1}\right]\left[\Sigma^{-1}\right]$ induces a natural equivalence

$$
K(\mathcal{C}) \longrightarrow K\left(\mathrm{~N}(\mathcal{C})\left[W^{-1}\right]\left[\Sigma^{-1}\right]\right) .
$$

Proof The additivity theorem implies that, for Waldhausen categories with factorization, the suspension endomorphism $\Sigma: \mathcal{C} \rightarrow \mathcal{C}$ induces - id: $K(\mathcal{C}) \rightarrow K(\mathcal{C})$. By naturality, we conclude that $\Sigma: \mathrm{N}(\mathcal{C})\left[W^{-1}\right] \rightarrow \mathrm{N}(\mathcal{C})\left[W^{-1}\right]$ acts invertibly on $K$-theory. Finally, since $K$-theory (viewed as a functor of small $\infty$-categories with finite colimits and a zero object and right-exact functors) preserves filtered colimits, we see that

$$
K\left(\mathrm{~N}(\mathcal{C})\left[W^{-1}\right]\left[\Sigma^{-1}\right]\right) \simeq \operatorname{colim} K\left(\mathrm{~N}(\mathcal{C})\left[W^{-1}\right]\right) \simeq K\left(\mathrm{~N}(\mathcal{C})\left[W^{-1}\right]\right) \simeq K(\mathcal{C}),
$$

where the last equivalence follows from Corollary 7.12.

Remark 9.33 On 0 -connective covers there is an equivalence $K(\mathcal{C})_{>0} \simeq K(\mathcal{C})_{>0}$ between this notion of nonconnective $K$-theory and the usual connective $K$-theory of $\mathcal{C}$. In degree 0 , there an isomorphism $\pi_{0} K(\mathcal{C}) \cong \pi_{0} K(\mathcal{C})$ if the underlying $\infty$-category of $\mathcal{C}$ is idempotent complete.

Theorem 9.34 Let $\mathcal{A} \rightarrow \mathcal{B} \rightarrow \mathcal{C}$ be a sequence of DHKS-saturated Waldhausen categories with factorization such that

$$
\mathrm{Ho}\left(\mathrm{N}(\mathcal{A})\left[W^{-1}\right]\left[\Sigma^{-1}\right]\right) \longrightarrow \operatorname{Ho}\left(\mathrm{N}(\mathcal{B})\left[W^{-1}\right]\left[\Sigma^{-1}\right]\right) \longrightarrow \operatorname{Ho}\left(\mathrm{N}(\mathcal{C})\left[W^{-1}\right]\left[\Sigma^{-1}\right]\right)
$$


is a localization sequence of triangulated categories. Then the induced map

$$
\boldsymbol{K}(\mathcal{A}) \longrightarrow \boldsymbol{K}(\mathcal{B}) \longrightarrow K(\mathcal{C})
$$

is a cofiber sequence of spectra.

Proof This follows from the natural equivalence $\boldsymbol{K}(-) \simeq \boldsymbol{K}\left(\mathrm{N}(-)\left[W^{-1}\right]\left[\Sigma^{-1}\right]\right)$ and the fact that cofiber sequence

$$
\boldsymbol{K}\left(\mathrm{N}(\mathcal{A})\left[W^{-1}\right]\left[\Sigma^{-1}\right]\right) \longrightarrow \boldsymbol{K}\left(\mathrm{N}(\mathcal{B})\left[W^{-1}\right]\left[\Sigma^{-1}\right]\right) \longrightarrow \boldsymbol{K}\left(\mathrm{N}(\mathcal{C})\left[W^{-1}\right]\left[\Sigma^{-1}\right]\right)
$$

is a cofiber sequence because $\boldsymbol{K}(-)$ is a localizing invariant.

\subsection{Extending corepresentability}

In this section, we show how to extend the corepresentability of negative $K$-theory obtained in Theorem 9.8 to maps out of any dualizable object, using the theory developed in Section 3. We begin with the following technical lemma:

Lemma 9.35 Let $\mathcal{B}$ be a small idempotent-complete stable $\infty$-category. Then the functor given by $(-) \widehat{\otimes} \mathcal{B}$ preserves equivalences, filtered colimits, the point and exact sequences.

Proof It follows from the definition that $(-) \widehat{\otimes} \mathcal{B}$ preserves equivalences, filtered colimits and the point. The characterization of $[48,6.3 .1 .16]$ implies that it preserves exact sequences.

We can now prove the main theorem of this section:

Theorem 9.36 Let $\mathcal{B}$ be a smooth and proper small stable $\infty$-category in the sense of Definitions 3.4 and 3.5. Then $\mathcal{U}_{\text {loc }}(\mathcal{B})$ is compact in $\mathcal{M}_{\text {loc }}$ and for every small stable $\infty$-category $\mathcal{A}$, we have a natural equivalence of spectra,

$$
\operatorname{Map}\left(\mathcal{U}_{\text {loc }}(\mathcal{B}), \mathcal{U}_{\text {loc }}(\mathcal{A})\right) \simeq \boldsymbol{K}\left(\mathcal{B}^{\text {op }} \widehat{\otimes} \mathcal{A}\right)
$$

Proof For any small idempotent-complete stable $\infty$-category $\mathcal{B}$, we can consider the functor

$$
(-) \widehat{\otimes} \mathcal{B}: \mathrm{Cat}_{\infty}^{\text {perf }} \longrightarrow \mathrm{Cat}_{\infty}^{\text {perf }} .
$$

By Lemma 9.35, the composed morphism

$$
\mathrm{Cat}_{\infty}^{\mathrm{perf}} \stackrel{(-) \widehat{\otimes} \mathcal{B}}{\longrightarrow} \mathrm{Cat}{ }_{\infty}^{\mathrm{perf}} \stackrel{\mathcal{U}_{\text {loc }}}{\longrightarrow} \mathcal{M}_{\mathrm{loc}}
$$


is a localizing invariant. Thus, we obtain a commutative diagram

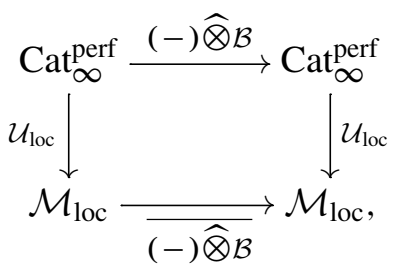

with $\overline{(-) \widehat{\otimes} \mathcal{B}}$ a colimit-preserving functor such that

$$
\overline{\mathcal{U}_{\mathrm{loc}}(\mathcal{A}) \widehat{\otimes} \mathcal{B}} \simeq \mathcal{U}_{\mathrm{loc}}(\mathcal{A} \widehat{\otimes} \mathcal{B}) .
$$

Now, recall from Theorem 3.7 that since $\mathcal{B}$ is smooth and proper, it is also dualizable (in the symmetric monoidal $\infty$-category $\mathrm{Cat}_{\infty}^{\text {perf }}$ of idempotent-complete small stable $\infty$-categories). Therefore, we have an adjunction (on the left) [48, 4.2.5.6], which induces an adjunction (on the right):

$$
\begin{array}{cc}
\mathrm{Cat}_{\infty}^{\text {perf }} & \mathcal{M}_{\text {loc }} \\
\left.(-) \widehat{\otimes} \mathcal{B} \downarrow\right|_{\mathrm{Cat}_{\infty}^{\text {perf }},} ^{\uparrow}(-) \widehat{\otimes} \mathcal{B}^{\text {op }} & \overline{(-) \widehat{\otimes} \mathcal{B}} \downarrow||_{(-) \widehat{\otimes} \mathcal{B}^{\text {op }}} \\
\mathcal{M}_{\text {loc }},
\end{array}
$$

with $\overline{(-) \widehat{\otimes} \mathcal{B}^{\mathrm{op}}}$ a colimit preserving morphism, such that

$$
\overline{\mathcal{U}_{\text {loc }}(\mathcal{A}) \widehat{\otimes} \mathcal{B}^{\mathrm{op}}} \simeq \mathcal{U}_{\text {loc }}\left(\mathcal{A} \widehat{\otimes} \mathcal{B}^{\mathrm{op}}\right) .
$$

The proof now follows from the following equivalences of spectra:

$$
\begin{aligned}
\operatorname{Map}\left(\mathcal{U}_{\mathrm{loc}}(\mathcal{B}), \mathcal{U}_{\mathrm{loc}}(\mathcal{A})\right) & \simeq \operatorname{Map}\left(\overline{\mathcal{U}_{\mathrm{loc}}\left(\mathcal{S}_{\infty}^{\omega}\right) \widehat{\otimes} \mathcal{B}}, \mathcal{U}_{\mathrm{loc}}(\mathcal{A})\right) \\
& \simeq \operatorname{Map}\left(\mathcal{U}_{\mathrm{loc}}\left(\mathcal{S}_{\infty}^{\omega}\right), \overline{\mathcal{U}_{\mathrm{loc}}(\mathcal{A}) \widehat{\otimes} \mathcal{B}^{\mathrm{op}}}\right) \\
& \simeq \operatorname{Map}\left(\mathcal{U}_{\mathrm{loc}}\left(\mathcal{S}_{\infty}^{\omega}\right), \mathcal{U}_{\mathrm{loc}}\left(\mathcal{A} \widehat{\otimes} \mathcal{B}^{\mathrm{op}}\right)\right) \\
& \simeq \boldsymbol{K}\left(\mathcal{A} \widehat{\otimes} \mathcal{B}^{\mathrm{op}}\right) \\
& \simeq \boldsymbol{K}\left(\operatorname{Fun}^{\mathrm{ex}}(\mathcal{B}, \operatorname{Idem}(\mathcal{A}))\right) .
\end{aligned}
$$

Finally, since in the adjunction

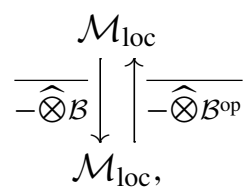


the morphism $\overline{(-) \widehat{\otimes} \mathcal{B}^{\mathrm{op}}}$ preserves colimits, the object $\mathcal{U}_{\text {loc }}\left(\mathcal{S}_{\infty}^{\omega}\right)$ is compact and $\mathcal{U}_{\text {loc }}\left(\mathcal{S}_{\infty}^{\omega}\right) \widehat{\otimes} \mathcal{B} \simeq \mathcal{U}_{\text {loc }}(\mathcal{B})$, we conclude that $\mathcal{U}_{\text {loc }}(\mathcal{B})$ is compact.

\subsection{Nonconnective $K$-theory of connective ring spectra}

In this section, we show that for a connective ring spectrum $R$, the nonconnective $K$-theory spectrum we associate to the category of perfect $R$-modules has negative homotopy groups determined by the classical nonconnective $K$-theory spectrum of the ring $\pi_{0} R$.

We give a proof using a model of nonconnective $K$-theory for connective ring spectra based on the construction of a "suspension ring spectrum" coupled with Quillen's plus construction. We begin by recalling Wagoner's construction [76] of the nonconnective $K$-theory of an ordinary ring $R$. Given a ring $R$, we let $\ell R$ denote the ring of locally finite (countably) infinite matrices in $R$; ie $\mathbb{N} \times \mathbb{N}$ matrices such that each row and column only has finitely many nonzero elements. We let $m R$ denote the finite matrices, regarded as a 2 -sided ideal of $\ell R$; these are the matrices with only finitely many nonzero elements. Then we can form the quotient ring $\mu R=\ell R / m R$, and Wagoner defines the nonconnective $K$-theory spectrum to have $n$-th space

$$
K(R)_{n}=K_{0}\left(\mu^{n} R\right) \times B \mathrm{GL}^{+}\left(\mu^{n} R\right) .
$$

It is known that this construction agrees with other possible constructions of the nonconnective algebraic $K$-theory spectrum of $R$ (eg see [58, Section 6]).

Next, we recall the generalization of this construction to connective ring spectra. Prior to the invention of modern notions of structured ring spectra, May initiated the study of the algebraic $K$-theory of a multiplicative object called an " $A_{\infty}$ ring space", which is an $E_{\infty}$ space with a suitably compatible $A_{\infty}$ multiplication (for a particular pair of operads) [50; 67]. The prototype example of an $A_{\infty}$ ring space is $\Omega^{\infty} R$ for a connective ring spectrum $R$ [50, 3.1]. Fiedorowicz, Schwänzl, Steiner and Vogt [31] extended Wagoner's constructions by defining $m R$ and $\ell R$ for $A_{\infty}$ ring spaces (using the work of [67] to define matrices with entries in $A_{\infty}$ ring spaces), and then defining $\mu R$ to be the homotopy cofiber of the inclusion $m R \rightarrow \ell R$. Furthermore, they prove that there is an equivalence of spaces

$$
K_{0}\left(\pi_{0}(R)\right) \times B \mathrm{GL}^{+}(R) \simeq \Omega\left(K_{0}\left(\pi_{0}(\mu R)\right) \times B \mathrm{GL}^{+}(\mu R)\right) .
$$

These constructions then allow a definition of the nonconnective algebraic $K$-theory of an $A_{\infty}$ ring space $R$ with spaces

$$
\boldsymbol{K}(R)_{n}=K_{0}\left(\mu^{n} \pi_{0} R\right) \times B \mathrm{GL}^{+}\left(\mu^{n} R\right) .
$$


This definition implies that for an $A_{\infty}$ ring space $R$, the natural map $R \rightarrow \pi_{0} R$ induces an isomorphism on the algebraic $K$-groups $K_{-n}(R):=K_{0}\left(\mu^{n} \pi_{0} R\right)$ for $n \geq 0$ [31, $1.1]$.

Our approach involves constructing a variant of the "suspension ring" construction that allows a construction of a nonconnective $K$-theory spectrum which agrees with the nonconnective $K$-theory of the ring space $\Omega^{\infty} R$ as defined in Equation (9.38) on $\pi_{i}$ for $i<0$ and is equivalent to our version of the nonconnective $K$-theory $\boldsymbol{K}(R):=\boldsymbol{K}\left(\widehat{R}_{\text {perf }}\right)$ spectrum constructed in Definition 9.6. Since $\pi_{0} \Omega^{\infty} R \cong \pi_{0} R$, this equivalence implies the desired comparison.

We begin by recalling the definition of the plus construction introduced in [77], extended to $A_{\infty}$ ring spectra. For convenience, we model $A_{\infty}$ ring spectra as EKMM $S$-algebras. We write $M_{n} R=\operatorname{map}_{R}\left(R^{\vee n}, R^{\vee n}\right)$ for the space of $R$-module endomorphisms of (a cofibrant replacement of) $R^{\vee n}$, and write $\operatorname{GL}_{n}(R) \rightarrow M_{n}(R)$ for the full subspace of $R$-module automorphisms of $R^{\vee n}$; that is, we have a (homotopy) pullback of spaces

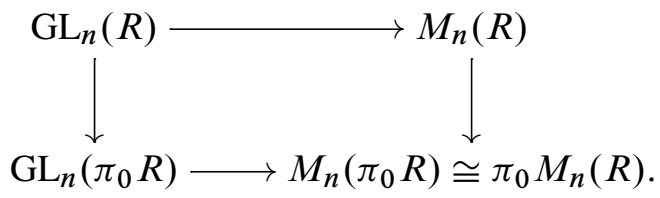

Since $\mathrm{GL}_{n}(R)$ is a topological monoid, after replacing to ensure the inclusion of the unit is a cofibration, we can form its classifying space $B \mathrm{GL}_{n}(R)$. Moreover, there are natural inclusions $\mathrm{GL}_{n}(R) \rightarrow \mathrm{GL}_{n+1}(R)$ which induce maps $B \mathrm{GL}_{n}(R) \rightarrow$ $B \mathrm{GL}_{n+1}(R)$. We can form

$$
B \mathrm{GL}(R) \cong \operatorname{hocolim}_{n} B \mathrm{GL}_{n}(R) .
$$

Since $\pi_{1} B \mathrm{GL}(R) \cong \mathrm{GL}\left(\pi_{0} R\right)$, we can form the plus construction $B \mathrm{GL}(R)^{+}$, and one could define the $K$-theory space to be the infinite loop space $K_{0}\left(\pi_{0} R\right) \times B G L(R)^{+}$. The consistency of this definition is proved in $[30,7.1]$, which we restate below:

Lemma 9.39 Let $R$ be a connective $A_{\infty}$ ring spectrum. There is an equivalence of infinite loop spaces

$$
\Omega^{\infty} K(R) \simeq K_{0}\left(\pi_{0} R\right) \times B \mathrm{GL}(R)^{+} .
$$

This is consistent in the sense that a check of the definition of the plus construction for an $A_{\infty}$ space [50, Section 7] now yields the following proposition: 
Proposition 9.40 For a connective ring spectrum $R$, the connective algebraic $K$ theory space $B \mathrm{GL}(R)^{+}$is equivalent to the algebraic $K$-theory space $B \mathrm{GL}^{+}\left(\Omega^{\infty} R\right)$.

We now set up analogues of the constructions of [31]. In order to ensure that our mapping spaces and spectra have the correct homotopy type, we continue to work with the category of EKMM algebra and module spectra. Since all objects are fibrant, it then suffices to work with cofibrant modules. For a connective ring spectrum $R$, in the following we let $\operatorname{Map}_{R}(x, y)$ denote the mapping spectrum between objects $x$ and $y$ and $\operatorname{map}_{R}(x, y)$ the mapping space (which can be computed as $\Omega^{\infty} \operatorname{Map}(x, y)$ ) in the category of $R$-modules. Moreover, when we write $R^{\vee n}$ inside a mapping object, we will tacitly mean the wedge of a cofibrant replacement of $R$ as an $R$-module.

Definition 9.41 Let $R$ be a connective $A_{\infty}$ ring spectrum. We set

$$
M R=\operatorname{colim}_{n} \operatorname{Map}_{R}\left(R^{\vee n}, R^{\vee n}\right),
$$

the nonunital $A_{\infty}$ ring spectrum of finite $R$-valued matrices. We write $L R$ for the $A_{\infty}$ ring spectrum of locally finite matrices, ie the connective $A_{\infty}$ ring spectrum obtained as the homotopy pullback

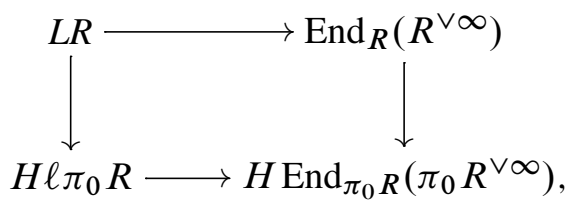

where here $H$ denotes the Eilenberg-Mac Lane spectrum functor.

Remark 9.42 These definitions are consistent with the those of $m$ and $\ell$ of [31] in the case of a ringlike $A_{\infty}$ space; one can construct equivalences $\Omega^{\infty} M R \simeq m\left(\Omega^{\infty} R\right)$ and $\Omega^{\infty} L R \simeq \ell\left(\Omega^{\infty} R\right)$, although we leave the details to the interested reader, in order avoid a detailed discussion of the technology for $A_{\infty}$ ring spaces.

We now begin to prove the Comparison Theorem 9.53 below. As explained in [14, Section 15], without loss of generality we can work with categories enriched in EKMM $S$-modules as a model for spectral categories, and we tacitly move between categories enriched in EKMM $S$-modules and categories enriched in symmetric spectra in the following discussion.

Let $F_{R}$ denote the spectral category of finitely generated free $R$-modules. The theorem follows from Proposition 9.51, which depends on the existence of a spectral category $\tilde{F}_{R}^{\infty}$, equipped with a homotopically fully faithful spectral functor $F_{R} \rightarrow \tilde{F}_{R}^{\infty}$, whose $\infty$-category of modules has a generator $G$ such that $\pi_{0}$ of the endomorphism ring 
spectrum of the image of $\mathrm{G}$ in the quotient category $\Psi\left(\tilde{F}_{R}^{\infty}\right) / \Psi\left(F_{R}\right)$ is $\mu\left(\pi_{0} \Omega^{\infty} R\right)$. This approach to constructing analogues of $\mu\left(\Omega^{\infty} R\right)$ is motivated by the explicit description of mapping spectra in the stable quotient (see [23, 1.3] for the dg-case and [14, Section 6] for the spectral analogue) and an idea from $[58,6.1]$.

We begin by giving a particular construction of such a spectral category. Roughly speaking, the idea is to adjoin the object $R^{\vee \infty}$ to $F_{R}$ in such as way that the inclusion $F_{R} \rightarrow \widetilde{F}_{R}^{\infty}$ is fully faithful and $\Psi\left(\widetilde{F}_{R}^{\infty}\right)$ is generated by an object $G=R^{\vee \infty}$ such that $\pi_{0}$ End $_{\tilde{F}^{\infty}}(G) \cong \ell\left(\pi_{0} \Omega^{\infty} R\right)$.

Recall that we denote by $\widehat{R}$ the category of $R$-modules, which we can regard as a spectral category. Let $F_{R}$ denote the full spectral subcategory of $\widehat{R}$ spanned by the finite free $R$-modules $R^{\vee n}, n \in \mathbb{N}$, and let $F_{R}^{\infty}$ denote the full spectral subcategory of $\widehat{R}$ spanned by the finite free $R$-modules as well as the countable wedge $R^{\vee \infty} \simeq$ $\operatorname{colim}_{n} R^{\vee n}$. The inclusion gives a fully faithful spectral functor $i: F_{R} \rightarrow F_{R}^{\infty}$. The spectral category $F_{R}^{\infty}$ is an intermediate construction that we will use to construct $\widetilde{F}_{R}^{\infty}$.

Write $\Psi\left(F_{R}\right)$ and $\Psi\left(F_{R}^{\infty}\right)$ for the presentable stable $\infty$-categories of $F_{R}$-modules and $F_{R}^{\infty}$-modules, and let $i_{!}: \Psi\left(F_{R}\right) \rightarrow \Psi\left(F_{R}^{\infty}\right)$ denote the left adjoint of the restriction $i^{*}: \Psi\left(F_{R}^{\infty}\right) \rightarrow \Psi\left(F_{R}\right)$. Given an $R$-algebra $A$, we will also write $\Psi(A)$ for the stable $\infty$-category of $A$-modules.

Proposition 9.43 The unit natural transformation $\mathrm{Id} \rightarrow i^{*} i$ ! is an equivalence.

Proof This is follows from the fact that $i_{!}: \Psi\left(F_{R}\right) \rightarrow \Psi\left(F_{R}^{\infty}\right)$ is fully faithful, which in turn follows from the fact that $i$ is a fully faithful functor of spectral categories.

Since $i$ ! is fully faithful, we have an exact sequence of presentable stable $\infty$-categories

$$
\Psi\left(F_{R}\right) \longrightarrow \Psi\left(F_{R}^{\infty}\right) \longrightarrow \mathcal{C}
$$

where $\mathcal{C}$ denotes the cofiber of $i_{\text {! }}$. We can regard $\mathcal{C}$ as the full subcategory of $\Psi\left(F_{R}^{\infty}\right)$ spanned by the local objects. In mild abuse of notation, for each $0 \leq n \leq \infty$, we will write $R^{\vee n}$ for the $F_{R}^{\infty}$-module represented by $R^{\vee n}$.

Proposition 9.44 The $F_{R}$-module represented by any finite wedge $R^{\vee n}$ is a compact generator of $\Psi\left(F_{R}\right)$ and the $F_{R}^{\infty}$-module represented by the countably infinite wedge $R^{\vee \infty}$ is a compact generator of $\Psi\left(F^{\infty} R\right)$. In particular, we have equivalences

$$
\Psi(R) \simeq \Psi\left(\operatorname{End}_{R}\left(R^{\vee n}\right)\right) \simeq \Psi\left(F_{R}\right) \text { and } \Psi\left(\operatorname{End}_{R}\left(R^{\vee \infty}\right)\right) \simeq \Psi\left(F_{R}^{\infty}\right)
$$


Proof The statement about compact generators essentially follows by construction. Then the $\infty$-categorical version of Schwede and Shipley's Morita Theorem [65], see also [48, Section 8.1.2], allows us to characterize these categories in terms of endomorphisms of the compact generator.

Since $i^{*} i_{\text {! }}$ is equivalent to the identity, the counit map $i_{!} i^{*} R^{\vee \infty} \rightarrow R^{\vee}$ restricts to an equivalence of $F_{R}$-modules. However, it is not an equivalence of $F_{R}^{\infty}$-modules, since not all endomorphisms of $R^{\vee \infty}$ (eg the identity) factor through $i_{!} i^{*} R^{\vee \infty}$.

The following proposition is standard; we restate it for convenience.

Proposition 9.45 An $F_{R}^{\infty}$-module $M$ is in the full subcategory $\mathcal{C} \subseteq \Psi\left(F_{R}^{\infty}\right)$ spanned by the local objects if and only if $i^{*} M \simeq 0$ in $\Psi\left(F_{R}\right)$. Similarly, a map of $F_{R}^{\infty}-$ modules $f: M \rightarrow M^{\prime}$ is a local equivalence if and only if the cofiber of $f$ lies in the essential image of $i_{\text {! }}$.

Proof The first claim follows from the fact that $i^{*} M \simeq 0$ if and only if for all $F_{R^{-}}$ modules $N, \operatorname{Map}_{F_{R}^{\infty}}(i ! N, M) \simeq 0$. In turn, this holds if and only if for any map of $F_{R}^{\infty}$-modules $Q \rightarrow P$ with cofiber of the form $i ! N$,

$$
\operatorname{Map}_{F_{R}^{\infty}}(P, M) \simeq \operatorname{Map}_{F_{R}^{\infty}}(Q, M) .
$$

The second claim follows from the fact that, if the cofiber of $f$ lies in the essential image of $i_{!}$, then for any local object $L, \operatorname{Map}_{F_{R}^{\infty}}\left(M^{\prime}, L\right) \simeq \operatorname{Map}_{F_{R}^{\infty}}(M, L)$.

As a consequence, we can identify a compact generator of $\mathcal{C}$.

Corollary 9.46 Let $G$ denote the cofiber of the counit $i_{!} i^{*} R^{\vee \infty} \rightarrow R^{\vee \infty}$ in $\Psi\left(F_{R}^{\infty}\right)$. Then $G$ lies in the full subcategory $\mathcal{C} \subseteq \Psi\left(F_{R}^{\infty}\right)$, ie $G$ is a local object, and the map $R^{\vee \infty} \rightarrow G$ is a local equivalence. Furthermore, $G$ is a compact generator of $\mathcal{C}$.

Proof By the previous proposition, $G$ is a local object, and the cofiber

$$
\Sigma i_{1} i^{*} R^{\vee \infty} \simeq i_{!} \Sigma i^{*} R^{\vee \infty}
$$

of $R^{\vee \infty} \rightarrow G$ is in the image of $i_{\text {! }}$. $G$ is compact because $R^{\vee \infty}$ is a compact generator of $F_{R}^{\infty}$ and the functor $\Psi\left(F_{R}^{\infty}\right) \rightarrow \mathcal{C}$ preserves compact objects [64, 2.9].

This suggests that we might consider $\operatorname{End}_{\mathcal{C}}(G)$, regarded as an $A_{\infty}$ ring spectrum under composition, as an analogue of $\hat{\mu} R$. Note that by Corollary 9.46, $\operatorname{End}_{\mathcal{C}}(G) \simeq$ $\operatorname{Map}_{F \infty}\left(R^{\vee \infty}, G\right)$ is equivalent to the cofiber (in spectra) of the map

$$
\operatorname{Map}_{F_{R}^{\infty}}\left(R^{\vee \infty}, i_{!} i^{*} R^{\vee \infty}\right) \longrightarrow \operatorname{Map}_{F_{R}^{\infty}}\left(R^{\vee \infty}, R^{\vee \infty}\right) \text {. }
$$


However, since $\pi_{0}\left(\operatorname{Map}_{F_{R}}\left(R^{\vee \infty}, R^{\vee \infty}\right)\right)$ can be identified as the collection of infinite matrices with values in $\pi_{0}(R)$ that have finitely many elements per row, we need to perform a construction analogous to Definition 9.41.

Let

$$
\pi_{0} \widetilde{F}_{R}^{\infty} \longrightarrow \pi_{0} F_{R}^{\infty}
$$

denote the subcategory of $\pi_{0} F_{R}^{\infty}$ consisting of those maps

$$
f \in \pi_{0} \operatorname{Map}\left(R^{\vee m}, R^{\vee n}\right) \cong \operatorname{Hom}_{\pi_{0} R}\left(\pi_{0} R^{\vee m}, \pi_{0} R^{\vee n}\right),
$$

for $0 \leq m, n \leq \infty$, which are locally finite when regarded as elements of the group of $\pi_{0} R$-valued $m \times n$-matrices. Since the composition induces on $\pi_{0}$ the product of matrices and products of locally finite matrices are locally finite, this specification does indeed define a subcategory of $\pi_{0} F_{R}^{\infty}$. Furthermore, $\pi_{0} \widetilde{F}_{R}^{\infty}$ inherits an enrichment over abelian groups from that of $\pi_{0} F_{R}^{\infty}$. We now perform a categorical analogue of Definition 9.41, using the Eilenberg-Mac Lane functor $H$ from categories enriched in abelian groups to spectral categories $[65,5.1 .5]$.

Lemma 9.47 The symmetric monoidal functor $\pi_{0}: \mathrm{Sp}_{\geq 0} \rightarrow \mathrm{Ab}$ is right adjoint to the Eilenberg-MacLane spectrum functor $H$, which is lax symmetric monoidal. It induces a functor

$$
\pi_{0}: \text { Cat }_{\mathrm{Sp}_{\geq 0}} \longrightarrow \mathrm{Cat}_{\mathrm{Ab}}
$$

from categories enriched in connective symmetric spectra to categories enriched in abelian groups with right adjoint $H$.

Using this we obtain a morphism of spectral categories

$$
H \pi_{0} \tilde{F}_{R}^{\infty} \longrightarrow H \pi_{0} F_{R}^{\infty} .
$$

Note that for $0 \leq m, n<\infty$, the induced map of Eilenberg-Mac Lane spectra

$$
\operatorname{Map}_{H \pi_{0} \widetilde{F}_{R}^{\infty}}\left(R^{\vee m}, R^{\vee n}\right) \longrightarrow \operatorname{Map}_{H \pi_{0} F_{R}^{\infty}}\left(R^{\vee m}, R^{\vee n}\right)
$$

is an equivalence, as finite matrices are locally finite.

We now define spectral categories $\widetilde{F}_{R}^{\infty}$ and $\widetilde{F}_{R}$ as the homotopy pullbacks

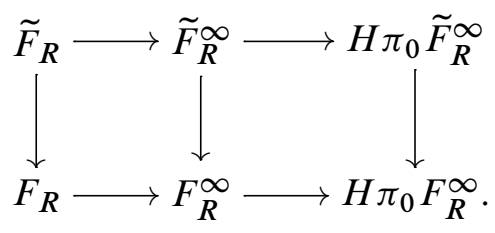


Observe that $\widetilde{F}_{R}$ and $\widetilde{F}_{R}^{\infty}$ have the same objects as $F_{R}$ and $F_{R}^{\infty}$, respectively, but $\operatorname{End} \widetilde{F}_{R}^{\infty}\left(R^{\vee \infty}\right) \simeq L R$.

Proposition 9.48 The spectral functor $\widetilde{F}_{R} \rightarrow F_{R}$ is a weak equivalence of spectral categories, and there is an equivalence of $A_{\infty}$ ring spectra $\operatorname{End} \widetilde{F}_{R}^{\infty}\left(R^{\vee \infty}\right) \simeq L R$.

Proof As the functor is actually surjective on objects, it is enough to show that it is fully faithful. This follows from the fact that mapping spectra in the homotopy pullback spectral category are computed as the homotopy pullbacks of the mapping spectra. Applying the long exact sequence to the homotopy pullback

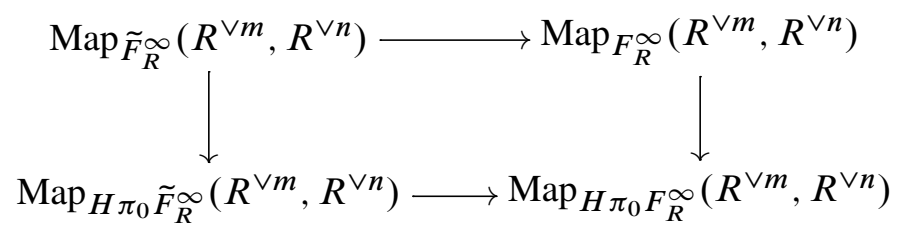

implies the desired equivalence. A similar computation with $m=n=\infty$ implies the second statement.

The spectral functor $\widetilde{F}_{R}^{\infty} \rightarrow F_{R}^{\infty}$ induces a functor (which is not fully faithful)

$$
\Psi\left(\tilde{F}_{R}^{\infty}\right) \longrightarrow \Psi\left(F_{R}^{\infty}\right)
$$

on $\infty$-categories of modules.

Carrying out the same analysis as above, we see that the quotient

$$
\mathcal{C}^{\prime}=\Psi\left(\widetilde{F}_{R}^{\infty}\right) / \Psi\left(F_{R}\right)
$$

can be described as modules over $\operatorname{End}_{\mathcal{C}^{\prime}}\left(G^{\prime}\right)$, where $G^{\prime}$ is the cofiber of the map

$$
i_{!} i^{*} R^{\vee \infty} \longrightarrow R^{\vee \infty}
$$

(here $R^{\vee \infty}$ is regarded as an object of $\left.\widetilde{F}_{R}^{\infty}\right)$ and hence as a spectrum $\operatorname{End}_{\mathcal{C}^{\prime}}\left(G^{\prime}\right)$ is equivalent to the cofiber in spectra of the map

$$
\operatorname{Map}_{\Psi\left(\tilde{F}_{R}^{\infty}\right)}\left(R^{\vee \infty}, i_{i} i^{*} R^{\vee \infty}\right) \longrightarrow \operatorname{End}_{\Psi\left(\tilde{F}_{R}^{\infty}\right)}\left(R^{\vee \infty}\right) .
$$

Lemma 9.50 There is an equivalence of rings $\pi_{0}\left(\operatorname{End}_{\mathcal{C}^{\prime}}\left(G^{\prime}\right)\right) \simeq \pi_{0}\left(\mu \Omega^{\infty} R\right)$.

Proof Regarding $\widetilde{F}_{R}^{\infty}$ as a simplicial category, $\operatorname{End} \widetilde{F}_{R}^{\infty}\left(R^{\infty}\right)$ is (by construction) the $A_{\infty}$ ring space $\ell R$. Furthermore, we have that

$$
\pi_{0}\left(\operatorname{Map}_{\Psi\left(\tilde{F}_{R}^{\infty}\right)}\left(R^{\vee \infty}, i_{!} i^{*} R^{\vee \infty}\right)\right) \cong \pi_{0}\left(i_{!} i^{*} R^{\vee \infty}\right) \cong m \pi_{0} R
$$


and by construction

$$
\pi_{0}\left(\operatorname{End}_{\Psi\left(\tilde{F}_{R}^{\infty}\right)}\left(R^{\vee \infty}\right)\right) \cong \ell \pi_{0} R .
$$

Therefore, Equation (9.49) implies that as groups there is an isomorphism

$$
\begin{aligned}
\pi_{0}\left(\operatorname{End}_{\mathcal{C}^{\prime}}\left(G^{\prime}\right)\right) \cong \ell\left(\pi_{0}(R)\right) / m\left(\pi_{0}(R)\right) & \cong \ell\left(\pi_{0}\left(\Omega^{\infty} R\right)\right) / m\left(\pi_{0}\left(\Omega^{\infty} R\right)\right) \\
& \cong \mu \pi_{0}\left(\Omega^{\infty} R\right) \cong \pi_{0}\left(\mu \Omega^{\infty} R\right),
\end{aligned}
$$

where the last isomorphism follows from $[31,5.1]$. Finally, the universal property of the cofiber in spectra implies that there is a ring structure induced on $\pi_{0}\left(\operatorname{End}_{\mathcal{C}^{\prime}}\left(G^{\prime}\right)\right)$ induced by the ring structure on $m \pi_{0}(R)$ quotiented by the two-sided ideal $\ell \pi_{0}(R)$. Inspection of $\pi_{0}$ shows that this multiplication coincides with the ring structure on $\pi_{0}\left(\operatorname{End}_{\mathcal{C}^{\prime}}\left(G^{\prime}\right)\right)$ induced by composition.

Based on this, we define

$$
\widehat{\mu} R \cong \operatorname{End}_{\mathcal{C}^{\prime}}\left(G^{\prime}\right),
$$

using the setup described above, and we proceed to relate this suspension ring spectrum construction to an $\infty$-categorical delooping. The basic idea is that our constructions of the suspension rings give (smaller) models of the $\infty$-categorical cone $\mathcal{F}_{K}$ from Definition 9.1 which are more closely related to the suspension ring spectrum $\hat{\mu} R$.

Proposition 9.51 Let $R$ be a connective $A_{\infty}$ ring spectrum. We have a natural equivalence of spectra

$$
K\left(\Psi_{\text {tri }}(\widehat{\mu} R)\right) \stackrel{\simeq}{\longrightarrow} K\left(\left(\operatorname{Ind}\left(\Psi_{\text {perf }}(R)\right)\right)^{\kappa} / \Psi_{\text {perf }} R\right)
$$

for any infinite cardinal $\kappa>\omega$.

Proof For any infinite cardinal $\kappa>\omega$, there is a natural inclusion map

$$
\Psi_{\text {perf }}\left(F_{R}^{\infty}\right) \longrightarrow\left(\operatorname{Ind}\left(\Psi_{\text {perf }}(R)\right)\right)^{\kappa}
$$

induced by the fact that any countable wedge of copies of $R$ is in $\left(\operatorname{Ind}\left(\Psi(R)_{\text {perf }}\right)\right)^{\kappa}$, and the latter is closed under retracts and stable under finite colimits. Since the inclusion $\Psi_{\text {perf }}\left(F_{R}\right) \rightarrow \Psi_{\text {perf }}\left(F_{R}^{\infty}\right)$ is compatible with the (Yoneda) inclusion $\Psi_{\text {perf }}(R) \rightarrow$ $\left(\operatorname{Ind}\left(\Psi_{\text {perf }}(R)\right)\right)^{\kappa}$, we have a commutative diagram

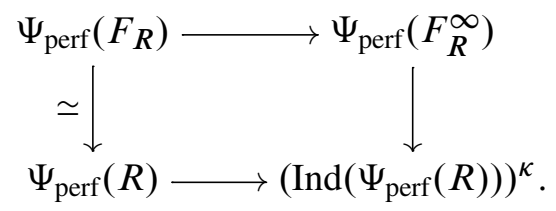


Combining this with $\Psi_{\text {perf }}\left(\widetilde{F}_{R}\right) \rightarrow \Psi_{\text {perf }}\left(\widetilde{F}_{R}^{\infty}\right)$ we obtain the commutative diagram

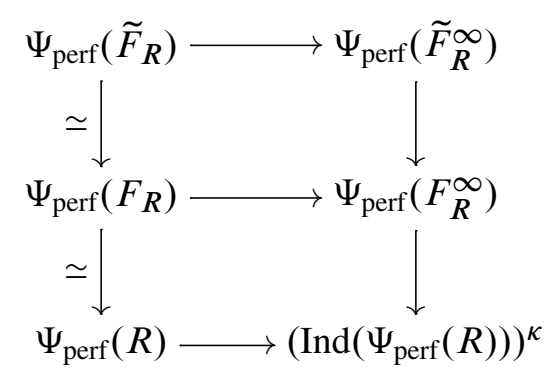

and hence an induced composite map of quotients

$\alpha: \Psi_{\text {perf }}\left(\widetilde{F}_{R}^{\infty}\right) / \Psi_{\text {perf }}\left(F_{R}\right) \longrightarrow \Psi_{\text {perf }}\left(F_{R}^{\infty}\right) / \Psi_{\text {perf }}\left(F_{R}\right) \longrightarrow\left(\operatorname{Ind}\left(\Psi_{\text {perf }}(R)\right)\right)^{\kappa} / \Psi_{\text {perf }}(R)$.

By the work above, $\alpha$ can be described as a map

$$
\Psi_{\text {tri }}(\widehat{\mu} R) \longrightarrow\left(\operatorname{Ind}\left(\Psi_{\text {perf }}(R)\right)\right)^{\kappa} / \Psi_{\text {perf }}(R) .
$$

Finally, since $F_{R}^{\infty}$ has countable coproducts, the usual Eilenberg swindle implies that $K\left(F_{R}^{\infty}\right)$ is contractible. We also know that $K\left(\Psi_{\text {perf }}\left(\tilde{F}_{R}^{\infty}\right)\right)$ is contractible [31, 6.1,6.3]. Therefore, applying $\operatorname{Map}\left(\underline{\mathcal{U}}_{\text {wloc }}^{\kappa}\left(\mathcal{S}_{\infty}^{\omega}\right), \underline{\mathcal{U}}_{\text {wloc }}^{\kappa}(-)\right)$ to the commutative diagram, the fact that all of the horizontal sequences are strict-exact allows us to apply Theorem 9.10 to conclude that $\alpha$ induces an equivalence on $K$-theory spectra.

Proposition 9.51 allows us finally to establish the desired result.

Theorem 9.53 Let $R$ be a connective $A_{\infty}$ ring spectrum. Then for $i \leq 0$, the natural map $R \rightarrow H \pi_{0} R$ induces isomorphisms $\pi_{i} K(R) \rightarrow \pi_{i} K\left(H \pi_{0} R\right) \cong \pi_{i} K\left(\pi_{0} R\right)$.

Proof Using the proof of Proposition 9.51 and mimicking Definition 9.6, we can define a spectrum

$$
\boldsymbol{K}^{\prime}(R):=\operatorname{colim}_{n} \Omega^{n} K\left(\Psi_{\text {tri }}\left(\hat{\mu}^{n} R\right)\right) .
$$

The conclusion of Proposition 9.51 along with diagram (9.52) (which implies compatibility of the structure maps) yields an equivalence $\boldsymbol{K}^{\prime}(R) \simeq \boldsymbol{K}(R)$. By the argument for $\left[64,11.7\right.$, we see that we can compute the homotopy groups of $\boldsymbol{K}^{\prime}(R)$ using a fibrant model that is a spectrum with $n$-th space given by the space

$$
\Omega^{\infty} K\left(\Psi_{\text {perf }}\left(\hat{\mu}^{n} R\right)\right) \text {. }
$$

Lastly, Lemmas 9.39 and 9.50 imply that there is an equivalence

$$
\Omega^{\infty} K\left(\Psi_{\text {perf }}\left(\widehat{\mu}^{n} R\right)\right) \simeq K_{0}\left(\mu^{n} \pi_{0} R\right) \times B \mathrm{GL}^{+}\left(\widehat{\mu}^{n} R\right) .
$$

Therefore, for $n>1, \pi_{0} \Omega^{\infty} K\left(\Psi_{\text {perf }}\left(\hat{\mu}^{n} R\right)\right)$ is $K_{0}\left(\mu^{n} \pi_{0} R\right)=K_{-n}\left(\pi_{0} R\right)$. 


\section{Trace maps}

In this section we apply the work of the preceding sections to give a universal characterization of the topological Dennis trace map $K \rightarrow T H H$ [16] and the cyclotomic trace map $K \rightarrow T C$ [17]. More generally, we identify all natural transformations of additive functors from $K$-theory to $T H H$ : they are the multiples of the topological Dennis trace map. This identification provides a very satisfying conceptual construction of the cyclotomic trace map, and of course makes it clear that all known definitions are consistent.

\subsection{THH as a localizing invariant}

We begin by observing that $T H H$ provides a localizing invariant of small stable $\infty-$ categories. Although it is possible to do this directly in the setting of $\infty$-categories (see for instance the more general discussion of topological chiral homology in [48, Section 5.3] or the constructions outlined in [6, 5.1.1]), we use existing constructions in the setting of spectral categories in order to ease technical difficulties that arise in the subsequent construction of $T R$ and $T C$. Our basic sources for this material are [14] and [10].

Recall that for a small spectral category $\mathcal{C}$ we can define $T H H(\mathcal{C})$ in terms of the Hochschild-Mitchell cyclic nerve for spectral categories [14, Section 3]. The cyclic nerve is defined as the simplicial object

$$
N_{q}^{\mathrm{cyc}} \mathcal{C}=\bigvee \mathcal{C}\left(c_{q-1}, c_{q}\right) \wedge \cdots \wedge \mathcal{C}\left(c_{0}, c_{1}\right) \wedge \mathcal{C}\left(c_{q}, c_{0}\right),
$$

where the sum is over the $(q+1)$-tuples $\left(c_{0}, \ldots, c_{q}\right)$ of objects of $\mathcal{C}$. This becomes a simplicial object using the usual cyclic bar construction face and degeneracy maps: The unit maps of $\mathcal{C}$ induce the degeneracy maps, and the composition maps in $\mathcal{C}$ (along with the twist map at the end) induce the face maps. We denote the geometric realization as $N^{\text {cyc }} \mathcal{C}$.

The spectrum $N^{\text {cyc }} \mathcal{C}$ has the correct homotopy type only when $\mathcal{C}$ has cofibrant mapping spectra $[14,3.1]$. Since the cofibrant objects in the Morita model structure on small spectral categories reviewed in Theorem 2.2 have cofibrant mapping spectra [69, 4.18], we can define the functor

$$
T H H:=N^{\mathrm{cyc}} \circ Q: \mathrm{Cat}_{\mathcal{S}} \longrightarrow \mathcal{S},
$$

where $Q$ denotes the cofibrant replacement functor in the Morita model structure on $\mathrm{Cat}_{\mathcal{S}}$. Since this construction preserves Morita equivalences [14, 5.12] (and in fact DK-equivalences $[14,5.9]$ ) the functor descends to the level of $\infty$-categories. 
Lemma 10.1 The functor

$$
T H H: \mathrm{Cat}_{\mathcal{S}} \longrightarrow \mathcal{S}
$$

induces a functor of $\infty$-categories

$$
T H H: \mathrm{Cat}_{\infty}^{\mathrm{ex}} \simeq \mathrm{N}\left(\left(\mathrm{Cat}_{\mathcal{S}}\right)^{\mathrm{c}}\right)\left[W^{-1}\right] \longrightarrow \mathrm{N}\left((\mathcal{S})^{\mathrm{c}}\right)\left[W^{-1}\right] \simeq \mathcal{S}_{\infty}
$$

This definition of THH as a functor of $\infty$-categories lets us deduce the following proposition from known properties of $T H H$ in the setting of spectral categories.

Proposition 10.2 THH is a localizing invariant of small stable $\infty$-categories.

Proof The cyclic bar construction commutes with filtered homotopy colimits of spectral categories. Furthermore, THH( - ) takes exact sequences of spectral categories to exact sequences of spectra $[14,7.1]$. Therefore, the induced functor on $\infty$-categories is a localizing invariant.

The force of the corepresentability result for algebraic $K$-theory (Theorem 7.13 ) is that it implies, via the spectral Yoneda Lemma, the following identification of the spectrum of natural transformations of additive functors $K \rightarrow E$.

Theorem 10.3 Given an additive invariant $E$ : $\mathrm{Cat}_{\infty}^{\mathrm{ex}} \longrightarrow \mathcal{S}_{\infty}$ with values in the stable $\infty$-category of spectra, we have a natural equivalence

$$
\operatorname{Nat}(K, E) \simeq E\left(\mathcal{S}_{\infty}^{\omega}\right),
$$

where $\operatorname{Nat}(K, E)$ denotes the spectrum of natural transformations from $K$ to $E$ as additive invariants from small stable $\infty$-categories to spectra.

Proof By Theorem 6.10, we can describe the additive invariants $K$ and $E$ as elements of $\operatorname{Fun}^{\mathrm{L}}\left(\mathcal{M}_{\mathrm{add}}, \mathcal{S}_{\infty}\right)$. The equivalence

$$
\operatorname{Nat}\left(\operatorname{Map}\left(\mathcal{U}_{\text {add }}\left(\mathcal{S}_{\infty}^{\omega}\right),-\right), E\right) \simeq E\left(\mathcal{S}_{\infty}^{\omega}\right)
$$

follows from 7.13 and the spectral Yoneda Lemma.

In particular, applying Theorem 10.3 to $\mathrm{THH}$ yields the following corollary.

Corollary 10.4 We have an equivalence of spectra

$$
\operatorname{Nat}(K(-), T H H(-)) \cong T H H\left(\mathcal{S}^{\omega}\right) \simeq T H H(\mathbb{S}) \simeq \mathbb{S} .
$$

Passing to $\pi_{0}$ on both sides we obtain an isomorphism between homotopy classes of natural transformations and $\pi_{0}(\mathbb{S}) \cong \mathbb{Z}$. 


\subsection{The topological Dennis trace map}

Next, we want to characterize the topological Dennis trace $K \rightarrow T H H$ in terms of the classification of Corollary 10.4. To do this, we briefly recall the construction of the topological Dennis trace map for spectral categories and verify that it descends to provide a natural transformation of additive invariants from $\mathrm{K}$-theory to $\mathrm{THH}$ on $\infty$-categories. We rely on the work of [14, Section 5].

Recall that any small spectral category $\mathcal{A}$ is equivalent in the Morita model structure to the small spectral category $\widehat{\mathcal{A}}_{\text {perf }}$ of perfect modules. The category $\widehat{\mathcal{A}}_{\text {perf }}$ admits the structure of a Waldhausen category by restriction from the spectral model structure on $\widehat{\mathcal{A}}$. As explained in [14, Section 15], without loss of generality we can work with categories enriched in EKMM $S$-modules. In this case, because all objects are fibrant the weak equivalences of the Waldhausen structure on $\widehat{\mathcal{A}}_{\text {perf }}$ are compatible with the spectral enrichment in the sense of [10, Section 1].

Now, following [10, Section 5], we construct a trace using the perspective of [52] by "mixing" a cyclic bar construction and Waldhausen's $S_{\bullet}$ construction; this is definition $[10,5.12]$. Upon passage to underlying $\infty$-categories, we end up with a natural transformation of localizing invariants.

Lemma 10.5 The topological Dennis trace above induces a natural transformation of localizing invariants

$$
K \longrightarrow T H H .
$$

Proof It is clear from the construction of the trace described above that it descends to a natural transformation of functors of $\infty$-categories $K \rightarrow T H H$. One checks on each side that the trace commutes with filtered homotopy colimits of spectral categories, and so the result follows.

As a corollary, we know that there exists an element $x \in \mathbb{Z}$ such that $x$ corresponds to the homotopy class of the topological Dennis trace under the identification of Corollary 10.4. The following theorem identifies this element as the unit.

Theorem 10.6 The topological Dennis trace is (up to homotopy) the natural transformation given by the identity element $1 \in \pi_{0}(\operatorname{THH}(\mathbb{S})) \cong \pi_{0}(\mathbb{S}) \cong \mathbb{Z}$.

Proof Given a point $\phi$ in $\operatorname{Nat}(K(-), T H H(-))$ (a specific natural transformation, that is), we can describe the corresponding element in $\pi_{0}(\mathbb{S})$ as the homotopy class represented by the composite

$$
\mathbb{S} \longrightarrow \operatorname{Map}\left(\mathcal{U}_{\text {add }}\left(\mathcal{S}_{\infty}^{\omega}\right), \mathcal{U}_{\text {add }}\left(\mathcal{S}_{\infty}^{\omega}\right)\right) \simeq K(\mathbb{S}) \stackrel{\phi}{\longrightarrow} T H H(\mathbb{S}) \simeq \mathbb{S}
$$


where the first map picks out the identity map in $\operatorname{Map}\left(\mathcal{U}_{\text {add }}\left(\mathcal{S}_{\infty}^{\omega}\right), \mathcal{U}_{\text {add }}\left(\mathcal{S}_{\infty}^{\omega}\right)\right)$. There is also a classical map $i: \mathbb{S} \rightarrow K(\mathbb{S})$ constructed (for instance) as the canonical inclusion of the finite sets into finite spaces. Waldhausen's calculations [77, Section 5] imply that the homotopy class of $i$ is represented by $1 \in \pi_{0}(K(\mathbb{S}))$. On the other hand, since the identity map is the unit for the multiplication on $\pi_{0}\left(\operatorname{Map}\left(\mathcal{U}_{\text {add }}\left(\mathcal{S}_{\infty}^{\omega}\right), \mathcal{U}_{\text {add }}\left(\mathcal{S}_{\infty}^{\omega}\right)\right)\right)$ induced by the composition, it must also be represented by $1 \in \pi_{0}(K(\mathbb{S}))$. Finally, specializing to the case when $\phi$ is the topological Dennis trace, Waldhausen $[77,5.2]$ proves that the composite

$$
\mathbb{S} \stackrel{i}{\longrightarrow} K(\mathbb{S}) \longrightarrow T H H(\mathbb{S}) \simeq \mathbb{S}
$$

is homotopic to the identity.

\subsection{TC and the cyclotomic trace map}

Much of the interest in the topological Dennis trace comes from the fact that the $T H H$ spectrum comes with an additional equivariant structure (generalizing the classical connection between the cyclic bar construction of a space and the free loop space) which allows a refinement into a theory called TC, the topological cyclic homology. The topological Dennis trace lifts to a map

$$
K \longrightarrow T C
$$

called the cyclotomic trace map [17].

We will once again apply corepresentability and point-set models to characterize the cyclotomic trace. However, when attempting to apply our corepresentability results to $T C$, we run into certain obstacles. Although $T C$ is Morita invariant and satisfies localization [14], it does not preserve filtered colimits and is therefore not a localizing (or additive) invariant. Nonetheless, we can adapt our results to characterize the cyclotomic trace in this setting.

We begin by recalling the definition of $T C$ in the context of spectral categories. Our review is brief; once again, we refer the interested reader to [10, Section 5] and [14, Section 4] for an authoritative treatment. Fix a prime $p$. For a spectral category $\mathcal{C}$, we can realize $\operatorname{THH}(\mathcal{C})$ as a cyclotomic $S^{1}$-spectrum. It is convenient to use the Bokstedt model of $T H H$, which is reviewed in detail in [14, Section 4]. Since the Bokstedt model is naturally weakly equivalent to $N^{\text {cyc }} Q \mathcal{C}$ for any spectral category $\mathcal{C}[14,3.1]$, we can leverage the work above. A cyclotomic structure is additional structure on an equivariant spectrum arising from the self-equivalence $S^{1} / H \cong S^{1}$ (for finite $H \subset S^{1}$ ) that models the structure of the free loop space. 
Roughly speaking, what we have is a set of compatible maps

$$
\phi^{H} T \longrightarrow T
$$

for finite $H \subset S^{1}$. The equivariant structure allows us to consider the associated nonequivariant spectra

$$
T R^{n}(\mathcal{C})=T H H(\mathcal{C})^{C_{p^{n-1}}},
$$

the fixed points with respect to the induced $C_{p^{n-1}}$ action. The inclusion of fixed points and the cyclotomic structure give rise to maps $F$ and $R$, respectively,

$$
F, R: T R^{n} \longrightarrow T R^{n-1} \text {. }
$$

We define $T C^{n}(\mathcal{C})$ to be the homotopy equalizer

$$
\operatorname{holim}_{F, R} T R^{n}(\mathcal{C}) \longrightarrow T R^{n-1}(\mathcal{C}) .
$$

We then have that

$$
T C(\mathcal{C})=\operatorname{holim}_{n} T C^{n}(\mathcal{C}),
$$

where we form the homotopy limit over the maps induced by the restriction $R$; this definition is equivalent to the one originally given in [17].

The work of [14, Section 5] produces a description of $T H H(\mathcal{C})$ for a spectral category $\mathcal{C}$ as a cyclotomic spectrum, and hence constructions of $T R^{n}, T C^{n}$ and $T C$. Moreover, a cyclotomic trace map $K \rightarrow T C$ is constructed which arises from compatible maps $K \rightarrow T C^{n}$. As above, we import these constructions into the setting of $\infty$-categories. First, we have the following lemma:

Lemma 10.7 The functors

$$
T R^{n}, T C^{n}, T C: \mathrm{Cat}_{\mathcal{S}} \longrightarrow \mathcal{S}
$$

induce functors of $\infty$-categories

$$
T R^{n}, T C^{n}, T C: \mathrm{Cat}_{\infty}^{\mathrm{ex}} \simeq \mathrm{N}\left(\left(\mathrm{Cat}_{\mathcal{S}}\right)^{\mathrm{c}}\right)\left[W^{-1}\right] \longrightarrow \mathrm{N}\left((\mathcal{S})^{\mathrm{c}}\right)\left[W^{-1}\right] \simeq \mathcal{S}_{\infty} .
$$

Proof By [14, 3.9], maps which induce equivalences on $T H H$ induce equivalences on $T R^{n}, T C^{n}$ and $T C$. As a consequence, the result follows from Lemma 10.1.

Next, we observe that each of the objects $T C^{n}$ provides a localizing invariant.

Proposition 10.8 The functor $T C^{n}$ is a localizing invariant of stable $\infty$-categories with values in the stable $\infty$-category of spectra. 
Proof [14, Localization Theorem 7.1] implies that $\left\{T C^{n}(\mathcal{C})\right\}$ takes short exact sequences of spectral categories to short exact sequences of small stable $\infty$-categories. Thus, we need to show that $T R^{n}(\mathcal{C})$ preserve filtered colimits. We know this for $\mathrm{THH}$, and the result now follows inductively from consideration of the fundamental cofibration sequence (eg [36, 2.1.4])

$$
\operatorname{THH}(\mathcal{C})_{p^{n-1}} \longrightarrow T R^{n}(\mathcal{C}) \longrightarrow T R^{n-1}(\mathcal{C})
$$

(where the left-hand term denotes the homotopy orbit space) and the fact that homotopy orbits commute with filtered colimits.

The topological Dennis trace lifts through the constructions of $T C^{n}$, essentially by construction. Roughly speaking (see $[10,5.12]$ for a detailed construction), the trace is induced by an "inclusion of objects" map $S_{\bullet} \mathcal{C} \rightarrow \operatorname{THH}\left(S_{\bullet} \mathcal{C}\right)$, given by taking an object to its identity map in the 0 -skeleton. Since the trace lands in the fixed set with respect to the spacewise $S^{1}$-action on the cyclotomic $T H H$ spectrum, it is compatible with the maps $R$ and $F$ (see eg [37,1.2] for a more detailed discussion of this). Moreover, we can check that the trace descends to a natural transformation of localizing invariant using Lemma 10.5.

Lemma 10.9 The topological Dennis trace above induces a natural transformation of localizing invariants

$$
K \longrightarrow T C^{n}
$$

Furthermore, the cyclotomic trace $K \rightarrow T C$ provides a natural transformation in this setting which is assembled from natural transformations of localizing invariants.

Lemma 10.10 The natural transformations of localizing invariants

$$
K \longrightarrow T C^{n}
$$

induce a natural transformation of spectrum-valued functors

$$
K \longrightarrow T C .
$$

Although $T C=$ holim $T C^{n}$ is not itself a localizing invariant (it does not preserve filtered colimits in general), any natural transformation of functors $K \rightarrow T C$ is equivalent to the data of compatible maps to each $T C^{n}$. Therefore, if we consider the spectrum of natural transformations of functors to spectra from $K \rightarrow T C$ which restrict to localizing invariants on each component, the spectrum is given by the limit (in the $\infty$-category of spectra)

$$
\lim _{n} \operatorname{Nat}\left(K(-), T C^{n}(-)\right)
$$


Finally, this yields the following characterization of the cyclotomic trace.

Theorem 10.11 After $p$-completion, the set of homotopy classes of compatible localizing invariants $\left\{K \rightarrow T C^{n}\right\}$ is isomorphic to $\mathbb{Z}_{p}$. The cyclotomic trace is represented by $1 \in \mathbb{Z}_{p}$.

Proof Using Theorem 10.3 as in the proof of Corollary 10.4, we see that the spectrum of natural transformations $K \rightarrow T C$ which restrict to localizing invariants on each component can be computed as the limits (in the $\infty$-category of spectra)

$$
\lim _{n} \operatorname{Nat}\left(K(-), T C^{n}(-)\right) \simeq \lim _{n} T C^{n}(\mathbb{S})=T C(\mathbb{S}) .
$$

Completing at the prime $p$, recall that $T C(\mathbb{S}) \simeq \mathbb{S} \vee \Sigma C P_{-1}^{\infty}[63$, Section 1]. Since $\pi_{0}\left(\Sigma C P_{-1}^{\infty}\right)=0$, we deduce that the set of homotopy classes of compatible invariants is $\mathbb{Z}_{p}$. Furthermore, using the argument for Theorem 10.6 and passing to the limit, we can identify the class of the cyclotomic trace by understanding the homotopy class of the composite

$$
\mathbb{S} \longrightarrow K(\mathbb{S}) \longrightarrow T C(\mathbb{S}) \longrightarrow T H H(\mathbb{S}) \simeq \mathbb{S}
$$

(after $p$-completion). An elaboration of Waldhausen's results [77, Section 5] (see [17, Section 5] or [63, Section 1]) implies that this homotopy class is the identity (ie the unit splits the trace $T C(\mathbb{S}) \rightarrow T H H(\mathbb{S}) \simeq \mathbb{S}$, which gives the identification of $T C(\mathbb{S})$ above), and so using the work of Theorem 10.6 we again deduce that the cyclotomic trace is represented by the unit.

\section{References}

[1] J Adámek, J Rosický, Locally presentable and accessible categories, London Math. Soc. Lecture Note Ser. 189, Cambridge Univ. Press (1994) MR1294136

[2] C Barwick, On the algebraic $K$-theory of higher categories, I. The universal property of Waldhausen K-theory arXiv:1204.3607

[3] C Barwick, On left and right model categories and left and right Bousfield localizations, Homology, Homotopy Appl. 12 (2010) 245-320 MR2771591

[4] C Barwick, D M Kan, Relative categories: another model for the homotopy theory of homotopy theories, Indag. Math. 23 (2012) 42-68 MR2877401

[5] H Bass, Algebraic K-theory, W A Benjamin, New York (1968) MR0249491

[6] D Ben-Zvi, J Francis, D Nadler, Integral transforms and Drinfeld centers in derived algebraic geometry, J. Amer. Math. Soc. 23 (2010) 909-966 MR2669705

[7] J E Bergner, A model category structure on the category of simplicial categories, Trans. Amer. Math. Soc. 359 (2007) 2043-2058 MR2276611 
[8] J E Bergner, A survey of ( $\infty, 1)$-categories, from: "Towards higher categories", (J C Baez, J P May, editors), IMA Vol. Math. Appl. 152, Springer, New York (2010) 69-83 MR2664620

[9] A J Blumberg, D Gepner, G Tabuada, Uniqueness of the multiplicative cyclotomic trace arXiv:1103.3923

[10] A J Blumberg, MA Mandell, Localization for $T H H(k u)$ and the topological Hochschild and cyclic homology of Waldhausen categories arXiv:1111.4003

[11] A J Blumberg, M A Mandell, The localization sequence for the algebraic $K$-theory of topological K-theory, Acta Math. 200 (2008) 155-179 MR2413133

[12] A J Blumberg, MA Mandell, Algebraic K-theory and abstract homotopy theory, Adv. Math. 226 (2011) 3760-3812

[13] A J Blumberg, MA Mandell, Derived Koszul duality and involutions in the algebraic K-theory of spaces, J. Topol. 4 (2011) 327-342 MR2805994

[14] A J Blumberg, M A Mandell, Localization theorems in topological Hochschild homology and topological cyclic homology, Geom. Topol. 16 (2012) 1053-1120 MR2928988

[15] J M Boardman, R M Vogt, Homotopy invariant algebraic structures on topological spaces, Lecture Notes in Math. 347, Springer, Berlin (1973) MR0420609

[16] M Bökstedt, Topological Hochschild homology, preprint

[17] M Bökstedt, W C Hsiang, I Madsen, The cyclotomic trace and algebraic K-theory of spaces, Invent. Math. 111 (1993) 465-539 MR1202133

[18] A K Bousfield, The localization of spaces with respect to homology, Topology 14 (1975) 133-150 MR0380779

[19] D-C Cisinski, Invariance de la $K$-théorie par équivalences dérivées, J. K-Theory 6 (2010) 505-546 MR2746284

[20] D-C Cisinski, G Tabuada, Non-connective $K$-theory via universal invariants, Compos. Math. 147 (2011) 1281-1320 MR2822869

[21] D-C Cisinski, G Tabuada, Symmetric monoidal structure on non-commutative motives, J. $K$-Theory 9 (2012) 201-268 MR2922389

[22] G Cortiñas, A Thom, Bivariant algebraic K-theory, J. Reine Angew. Math. 610 (2007) 71-123 MR2359851

[23] V Drinfeld, DG quotients of DG categories, J. Algebra 272 (2004) 643-691 MR2028075

[24] D Dugger, Combinatorial model categories have presentations, Adv. Math. 164 (2001) 177-201 MR1870516

[25] D Dugger, D I Spivak, Mapping spaces in quasi-categories, Algebr. Geom. Topol. 11 (2011) 263-325 
[26] B I Dundas, Relative K-theory and topological cyclic homology, Acta Math. 179 (1997) 223-242 MR1607556

[27] W G Dwyer, D M Kan, Calculating simplicial localizations, J. Pure Appl. Algebra 18 (1980) 17-35 MR578563

[28] W G Dwyer, D M Kan, Simplicial localizations of categories, J. Pure Appl. Algebra 17 (1980) 267-284 MR579087

[29] W G Dwyer, D M Kan, Equivalences between homotopy theories of diagrams, from: "Algebraic topology and algebraic $K$-theory", (W Browder, editor), Ann. of Math. Stud. 113, Princeton Univ. Press (1987) 180-205 MR921478

[30] A D Elmendorf, I Kriz, M A Mandell, J P May, Rings, modules, and algebras in stable homotopy theory, Math. Surveys and Monographs 47, Amer. Math. Soc. (1997) MR1417719

[31] Z Fiedorowicz, R Schwänzl, R Steiner, R Vogt, Nonconnective delooping of $K$ theory of an $A_{\infty}$ ring space, Math. Z. 203 (1990) 43-57 MR1030707

[32] T Geisser, L Hesselholt, Topological cyclic homology of schemes, from: "Algebraic $K$-theory”, (W Raskind, C Weibel, editors), Proc. Sympos. Pure Math. 67, Amer. Math. Soc. (1999) 41-87 MR1743237

[33] T Geisser, L Hesselholt, On relative and bi-relative algebraic $K$-theory of rings of finite characteristic, J. Amer. Math. Soc. 24 (2011) 29-49

[34] P G Goerss, J F Jardine, Simplicial homotopy theory, Progress in Mathematics 174, Birkhäuser, Basel (1999) MR1711612

[35] T G Goodwillie, Cyclic homology, derivations, and the free loopspace, Topology 24 (1985) 187-215 MR793184

[36] L Hesselholt, Witt vectors of non-commutative rings and topological cyclic homology, Acta Math. 178 (1997) 109-141 MR1448712

[37] L Hesselholt, I Madsen, On the K-theory of local fields, Ann. of Math. 158 (2003) 1-113 MR1998478

[38] N Higson, A characterization of KK-theory, Pacific J. Math. 126 (1987) 253-276 MR869779

[39] PS Hirschhorn, Model categories and their localizations, Math. Surveys and Monographs 99, Amer. Math. Soc. (2003) MR1944041

[40] A Hirschowitz, C Simpson, Descente pour les n-champs arXiv:math/9807049

[41] M Hovey, B Shipley, J Smith, Symmetric spectra, J. Amer. Math. Soc. 13 (2000) 149-208 MR1695653

[42] A Joyal, Quasi-categories and Kan complexes, J. Pure Appl. Algebra 175 (2002) 207-222 MR1935979 
[43] A Joyal, M Tierney, Quasi-categories vs Segal spaces, from: "Categories in algebra, geometry and mathematical physics", (A Davydov, M Batanin, M Johnson, S Lack, A Neeman, editors), Contemp. Math. 431, Amer. Math. Soc. (2007) 277-326 MR2342834

[44] B Keller, On the cyclic homology of exact categories, J. Pure Appl. Algebra 136 (1999) 1-56 MR1667558

[45] H Krause, On Neeman's well generated triangulated categories, Doc. Math. 6 (2001) 121-126 MR1836046

[46] H Krause, Localization theory for triangulated categories, from: "Triangulated categories”, (T Holm, P Jørgensen, R Rouquier, editors), London Math. Soc. Lecture Note Ser. 375, Cambridge Univ. Press (2010) 161-235 MR2681709

[47] J Lurie, Higher topos theory, Annals of Mathematics Studies 170, Princeton Univ. Press (2009) MR2522659

[48] J Lurie, Higher algebra, preprint (2012) Available at http://tinyurl.com/ cdeqd7z

[49] M Makkai, R Paré, Accessible categories: the foundations of categorical model theory, Contemporary Mathematics 104, Amer. Math. Soc. (1989) MR1031717

[50] J P May, $A_{\infty}$ ring spaces and algebraic $K$-theory, from: "Geometric applications of homotopy theory, II", (M G Barratt, ME Mahowald, editors), Lecture Notes in Math. 658, Springer, Berlin (1978) 240-315 MR513580

[51] R McCarthy, On fundamental theorems of algebraic K-theory, Topology 32 (1993) 325-328 MR1217072

[52] R McCarthy, The cyclic homology of an exact category, J. Pure Appl. Algebra 93 (1994) 251-296 MR1275967

[53] R McCarthy, Relative algebraic $K$-theory and topological cyclic homology, Acta Math. 179 (1997) 197-222 MR1607555

[54] R Meyer, R Nest, The Baum-Connes conjecture via localisation of categories, Topology 45 (2006) 209-259 MR2193334

[55] F Morel, V Voevodsky, $\mathbf{A}^{1}$-homotopy theory of schemes, Inst. Hautes Études Sci. Publ. Math. (1999) 45-143 MR1813224

[56] A Neeman, The connection between the $K$-theory localization theorem of Thomason, Trobaugh and Yao and the smashing subcategories of Bousfield and Ravenel, Ann. Sci. École Norm. Sup. 25 (1992) 547-566 MR1191736

[57] A Neeman, Triangulated categories, Annals of Mathematics Studies 148, Princeton Univ. Press (2001) MR1812507

[58] E K Pedersen, C A Weibel, $K$-theory homology of spaces, from: "Algebraic topology”, (G Carlsson, R L Cohen, HR Miller, D C Ravenel, editors), Lecture Notes in Math. 1370, Springer, Berlin (1989) 346-361 MR1000388 
[59] D Quillen, Higher algebraic $K$-theory. I, from: "Algebraic $K$-theory, I: Higher $K-$ theories”, (H Bass, editor), Lecture Notes in Math. 341, Springer, Berlin (1973) 85-147 MR0338129

[60] C Rezk, A model for the homotopy theory of homotopy theory, Trans. Amer. Math. Soc. 353 (2001) 973-1007 MR1804411

[61] C Rezk, S Schwede, B Shipley, Simplicial structures on model categories and functors, Amer. J. Math. 123 (2001) 551-575 MR1833153

[62] J Rognes, A spectrum level rank filtration in algebraic $K$-theory, Topology 31 (1992) 813-845 MR1191383

[63] J Rognes, Two-primary algebraic $K$-theory of spaces and related spaces of symmetries of manifolds, from: "Algebraic $K$-theory", (W Raskind, C Weibel, editors), Proc. Sympos. Pure Math. 67, Amer. Math. Soc. (1999) 213-229 MR1743242

[64] M Schlichting, Negative K-theory of derived categories, Math. Z. 253 (2006) 97-134 MR2206639

[65] S Schwede, B Shipley, Stable model categories are categories of modules, Topology 42 (2003) 103-153 MR1928647

[66] C Simpson, A Giraud-type characterization of the simplicial categories associated to closed model categories as $\infty$-pretopoi arXiv:math/9903167

[67] R J Steiner, Infinite loop structures on the algebraic $K$-theory of spaces, Math. Proc. Cambridge Philos. Soc. 90 (1981) 85-111 MR611287

[68] G Tabuada, Higher K-theory via universal invariants, Duke Math. J. 145 (2008) 121-206 MR2451292

[69] G Tabuada, Homotopy theory of spectral categories, Adv. Math. 221 (2009) 1122-1143 MR2518634

[70] G Tabuada, Matrix invariants of spectral categories, Int. Math. Res. Not. 2010 (2010) 2459-2511 MR2669656

[71] Z Tamsamani, On non-strict notions of $n$-category and $n$-groupoid via multisimplicial sets arXiv:alg-geom/9512006

[72] R W Thomason, T Trobaugh, Higher algebraic $K$-theory of schemes and of derived categories, from: "The Grothendieck Festschrift, Vol. 3”, (P Cartier, L Illusie, N M Katz, G Laumon, K A Ribet, editors), Progr. Math. 88, Birkhäuser, Boston, MA (1990) 247-435 MR1106918

[73] B Toën, The homotopy theory of $d g$-categories and derived Morita theory, Invent. Math. 167 (2007) 615-667 MR2276263

[74] B Toën, G Vezzosi, A remark on $K$-theory and $S$-categories, Topology 43 (2004) 765-791 MR2061207 
[75] V Voevodsky, Triangulated categories of motives over a field, from: "Cycles, transfers, and motivic homology theories", Ann. of Math. Stud. 143, Princeton Univ. Press (2000) 188-238 MR1764202

[76] J B Wagoner, Delooping classifying spaces in algebraic K-theory, Topology 11 (1972) 349-370 MR0354816

[77] F Waldhausen, Algebraic K-theory of topological spaces. II, from: "Algebraic topology”, (J L Dupont, I H Madsen, editors), Lecture Notes in Math. 763, Springer, Berlin (1979) 356-394 MR561230

[78] F Waldhausen, Algebraic $K$-theory of spaces, from: "Algebraic and geometric topology”, (A Ranicki, N Levitt, F Quinn, editors), Lecture Notes in Math. 1126, Springer, Berlin (1985) 318-419 MR802796

Department of Mathematics, University of Texas at Austin

1 University Station C1200, Austin, TX 78712, USA

Fakultät für Mathematik, Universität Regensburg

D-93040 Regensburg, Germany

Department of Mathematics, Massachusetts Institute of Technology

77 Massachusetts Avenue, Cambridge, MA 02139, USA

and

Departamento de Matemática e CMA, FCT-UNL

Quinta da Torre, 2929-516 Caparica, Portugal

blumberg@math.utexas.edu, djgepner@gmail.com, tabuada@math.mit.edu

http://www . math . utexas. edu/users/blumberg/,

http://www.uni-regensburg.de/Fakultaeten/nat_Fak_I/gepner/,

http://math.mit.edu/ tabuada/

Proposed: Haynes Miller

Received: 1 January 2011

Seconded: Ralph Cohen, Paul Goerss

Revised: 2 October 2012 